\title{
DEBRIS DISKS IN THE UPPER SCORPIUS OB ASSOCIATION
}

\author{
John M. Carpenter ${ }^{1}$, Eric E. Mamajek ${ }^{2}$, Lynne A. Hillenbrand ${ }^{1}$, and Michael R. Meyer ${ }^{3}$ \\ ${ }^{1}$ California Institute of Technology, Department of Astronomy, MC 105-24, Pasadena, CA 91125, USA \\ ${ }^{2}$ University of Rochester, Department of Physics and Astronomy, Rochester, NY, 14627-0171, USA \\ 3 Institute for Astronomy, ETH, CH-8093 Zurich, Switzerland \\ Received 2009 July 12; accepted 2009 September 29; published 2009 October 26
}

\begin{abstract}
We present MIPS $24 \mu \mathrm{m}$ and $70 \mu \mathrm{m}$ photometry for 205 members of the Upper Scorpius OB Association. These data are combined with published MIPS photometry for 15 additional association members to assess the frequency of circumstellar disks around 5 Myr old stars with spectral types between B0 and M5. Twelve stars have a detectable $70 \mu \mathrm{m}$ excess, each of which also has a detectable $24 \mu \mathrm{m}$ excess. A total of 54 stars are identified with a $24 \mu \mathrm{m}$ excess more than $32 \%$ above the stellar photosphere. The MIPS observations reveal 19 excess sources-8 $\mathrm{A} / \mathrm{F} / \mathrm{G}$ stars and $11 \mathrm{~K} / \mathrm{M}$ stars-that were not previously identified with an $8 \mu \mathrm{m}$ or $16 \mu \mathrm{m}$ excess. The lack of short-wavelength emission and the weak $24 \mu \mathrm{m}$ excess suggests that these sources are debris systems or the remnants of optically thick primordial disks with inner holes. Despite the wide range of luminosities of the stars hosting apparent debris systems, the excess characteristics are consistent with all stars having dust at similar orbital radii after factoring in variations in the radiation blowout particle size with spectral type. The results for Upper Sco are compared to similar photometric surveys from the literature to re-evaluate the evolution of debris emission. After considering the completeness limits of published surveys and the effects of stellar evolution on the debris luminosity, we find that the magnitude of the $24 \mu \mathrm{m}$ excess around F-type stars increases between ages of 5 and 17 Myr as found by previous studies, but at $\lesssim 2.6 \sigma$ confidence. For B7-A9 and G0-K5 stars, any variations in the observed $24 \mu \mathrm{m}$ excess emission over this age range are significant at less than $2 \sigma$ confidence.
\end{abstract}

Key words: circumstellar matter - open clusters and associations: individual (Upper Scorpius) - planetary systems: formation - planetary systems: protoplanetary disks - stars: pre-main sequence

Online-only material: machine-readable tables

\section{INTRODUCTION}

Dusty debris observed around main-sequence stars is a potential diagnostic of planetary systems. The current paradigm is that the debris originates from the collisional grinding of planetesimals to micron-sized grains, where the collision rate is dictated by gravitational interactions between planets and a planetesimal belt (Williams \& Wetherill 1994). If this hypothesis is correct, the spatial asymmetries in the debris that have been revealed by high-resolution imaging (e.g., Holland et al. 1998) encodes information on the eccentricity, mass, and even migration of the orbiting planets (Liou \& Zook 1999; Wyatt 2003). Unfortunately, the resolution and sensitivity required to image the debris can be achieved for only a few disks with current instruments.

A complementary approach to detailed studies of individual disks is to study the ensemble properties of debris systems. Debris disks have been studied extensively over the entire sky using IRAS (Backman \& Paresce 1993; Lagrange et al. 2000; Rhee et al. 2007) and in targeted regions using the Infrared Space Observatory (ISO; Habing et al. 2001; Spangler et al. 2001; Dominik \& Decin 2003). The Spitzer Space Telescope (Werner et al. 2004) has expanded on these studies to produce a vast database of debris disks (e.g., Rieke et al. 2005; Bryden et al. 2006; Su et al. 2006; Gautier et al. 2007; Currie et al. 2008a; Carpenter et al. 2009, see also reviews by Werner et al. 2006, Meyer et al. 2007 and Wyatt 2008) that encompass a broad range of spectral types (B-M stars), environments (clusters, associations, field stars), and ages (3 Myr to $10 \mathrm{Gyr}$ ). Comparison of these data to theoretical models have yielded insights on the planetesimal belts that produce the debris dust
(Wyatt et al. 2007b), the collisional history of planetesimal belts (Dominik \& Decin 2003; Wyatt et al. 2007a; Löhne et al. 2008) and the formation of planetary systems (Kenyon \& Bromley 2008, and references therein).

A critical issue emerging from Spitzer studies is establishing when the debris phenomenon is initiated. In the Kenyon \& Bromley models, a planetesimal belt produces low levels of debris emission in the early stage of planetary accretion. At a given orbital radius, dust production reaches a maximum when 1000-3000 km sized bodies form and ignite the destructive collisional cascade (Kenyon \& Bromley 2004). As planet formation propagates through the disk, the disk is depleted of planetesimals through repeated collisions and the debris production eventually declines. In theory, the evolution of the debris emission can constrain the formation time of planets and the collisional cascade. Indeed, based on Spitzer observations, Hernández et al. (2006) and Currie et al. (2008a) have suggested that the debris luminosity peaks around 10-30 Myr for A-F stars before declining toward older ages (Rieke et al. 2005).

Additional observations are needed to validate previous conclusions since few surveys for debris emission at ages of $\lesssim 10$ Myr have been completed (Hernández et al. 2006; Carpenter et al. 2006; Gautier et al. 2008; Currie et al. 2009). The Upper Scorpius OB Association (hereafter, Upper Sco) is an important region in studies of disk evolution since it provides a snapshot of disk properties at an age of $\sim 5 \mathrm{Myr}$ when most optically thick disks have dissipated (Hernández et al. 2007). Spitzer observations can then probe the onset of debris production as planets form. Since association members have been identified from $\mathrm{B}$ to $\mathrm{M}$ stars, disk properties can be investigated over nearly two orders of magnitude in stellar mass. Finally, at 
a mean distance of 145 pc (de Zeeuw et al. 1999), Upper Sco is more than a factor of 2 closer than other clusters or associations of similar age. These traits permit sensitive photometric studies of debris properties.

In previous studies, we investigated the $4.5-16 \mu \mathrm{m}$ photometric (Carpenter et al. 2006, hereafter Paper I) and 5-35 $\mu \mathrm{m}$ spectroscopic (Dahm \& Carpenter 2009) properties of circumstellar disks in Upper Sco. The principal result from these studies is that the circumstellar disks detected at wavelengths shortward of $16 \mu \mathrm{m}$ show a dichotomy with spectral type. Circumstellar disks around late-type stars ( $\mathrm{K}$ and $\mathrm{M}$ spectral types) are likely optically thick primordial disks formed as a result of the star formation process, while early-type stars (B and A types) appear to be surrounded by debris disks. Surprisingly, no disks were detected around the $\mathrm{F}$ and $\mathrm{G}$ stars.

The photometric data presented in Paper I spanned wavelengths between $4.5 \mu \mathrm{m}$ and $16 \mu \mathrm{m}$. To probe for cooler dust, we present here $24 \mu \mathrm{m}$ and $70 \mu \mathrm{m}$ photometric observations of 205 Upper Sco members. These new Spitzer observations of Upper Sco are presented in Section 2. In Section 3, we use these data to identify stars that have excesses in the $24 \mu \mathrm{m}$ and $70 \mu \mathrm{m}$ bands. The nature of the excess sources is investigated in Section 4, and properties of debris systems around stars with a wide range in mass are compared in Section 5. In Section 6, we compare the Upper Sco results with published Spitzer surveys to investigate the evolution of debris systems.

\section{OBSERVATIONS AND DATA REDUCTION}

The selection criteria for the Upper Sco sample are described in Paper I. Briefly, the sample was constructed from membership lists established by Hipparcos astrometry (de Zeeuw et al. 1999), color-magnitude diagrams (Preibisch \& Zinnecker 1999; Preibisch et al. 2002), and X-ray surveys (Walter et al. 1994; Martín 1998; Preibisch et al. 1998; Kunkel 1999; Köhler et al. 2000). Since membership selection was based upon stellar properties (proper motions, photospheric colors, X-ray activity) unrelated to circumstellar disks, the sample is believed to be unbiased with respect to the presence or absence of disks. From the compiled list, we selected 205 stars $^{4}$ to sample spectral types between B0 and M5. This sample was supplemented with 15 Upper Sco members with $\mathrm{G} / \mathrm{K}$ spectral types that were observed by the Formation and Evolution of Planetary Systems (FEPSs) Spitzer Legacy program (Meyer et al. 2006). The FEPS targets were selected based on similar criteria as our study and should also be an unbiased sample with respect to the presence or absence of disks.

Other Upper Sco members have been observed with Spitzer but are not included in our sample. The Herbig Ae/Be star HIP 79476 (Hernández et al. 2005) was observed with the Spitzer Infrared Spectrograph (IRS), but not with the Multiband Imaging Photometer for Spitzer (MIPS). We did not include the G6/G8 star HD 143006, which was also observed by the FEPS program as part of survey of gas in disks. HD 143006 was recognized as a Upper Sco member based on an IRAS excess

\footnotetext{
4 Bouy \& Martín (2009) found that the star [PBB2002] USco 161021.5-194132 deviates from the mean proper motion for Upper Sco and may be an interloper. The reduced $\chi^{2}$ from the 2MASS point-spread function fitting photometry is the second highest among the nearest 100 stars to this source that have a $2 \mathrm{MASS}$ quality flag of AAA. The astrometry may therefore be contaminated by a binary. The luminosity of this star is also higher than the 10 nearest stars of the same spectral type (Preibisch et al. 2002), consistent with a binary contaminant. Given that the lithium equivalent width (Preibisch et al. 2002) and apparent brightness are consistent with Upper Sco membership, we retain the source in our sample.
}

(Odenwald 1986) and thus would bias the sample. We also do not use the three B stars observed by Rieke et al. (2005) and the five F/G stars observed by Chen et al. (2005) since IRAC data are not available for these sources, which is needed for the analysis presented in Section 3. Finally, we did not include brown-dwarfs in Upper Sco (Scholz et al. 2007).

In Paper I, IRAC (4.5 $\mu \mathrm{m}$ and $8 \mu \mathrm{m})$ and IRS peak-up $(16 \mu \mathrm{m})$ photometry was presented for 218 of the 220 stars. IRAC photometry for one additional Upper Sco member observed by FEPS (ScoPMS 52) is now available (Carpenter et al. 2008). The remaining star (HIP 80112) was not observed with IRAC since it would have saturated the detector. The $4.5 \mu \mathrm{m}$ flux density for this source was estimated from ground-based $M$-band observations (van der Bliek et al. 1996).

We present here MIPS $24 \mu \mathrm{m}$ and $70 \mu \mathrm{m}$ photometry for 205 stars in Upper Sco. The data reduction procedures follow that used by the FEPS program as described in Carpenter et al. (2008), and a only summary of the salient steps is provided here. Table 1 presents the $24 \mu \mathrm{m}$ and $70 \mu \mathrm{m}$ MIPS photometry for the 205 stars observed for this study. MIPS photometry for the remaining 15 stars are listed in Carpenter et al. (2008).

\subsection{MIPS $24 \mu \mathrm{m}$}

MIPS $24 \mu \mathrm{m}$ observations for 205 stars were obtained in photometry mode. The exposure time per image (either 3 or $10 \mathrm{~s}$ ) and the number of dithered images were set to achieve a signal-to-noise ratio of at least 10 on the expected photospheric brightness. MIPS $24 \mu \mathrm{m}$ images were processed starting with the Basic Calibrated Data (BCD) products produced by the Spitzer Science Center (SSC) pipeline version S17. Individual $\mathrm{BCD}$ images were combined to derive a flat field that removed long-term gain changes in the MIPS array. Flat field images were derived from stars that are not surrounded by nebulosity. If nebulosity is present, a flat-field image from another star was used that was observed close in time and with the same exposure time.

Point(source)-response-function (PRF) photometry was performed with the MOPEX package (Makovoz \& Marleau 2005). The empirical PRF distributed with MOPEX was fitted to the individual BCD images simultaneously (as opposed to the mosaicked image) using a fitting area of $21 \times 21$ pixels ( 1 pixel $\approx$ 2 .'55) for most sources. A $5 \times 5$ pixel fitting area was used for sources that have spatially variable nebulosity near the point source position. From visual inspection of the mosaicked images, the PRF of the Upper Sco target overlaps occasionally with that from a nearby source. These contaminating sources were fitted with a PRF simultaneously. The measured $24 \mu \mathrm{m}$ flux densities and internal uncertainties from the PRF fit are presented in Table 1. The images were processed with a calibration factor of 0.0447 $\mathrm{MJy} \mathrm{sr}^{-1}$. Following Engelbracht et al. (2007), we adopt a calibration uncertainty of $4 \%$.

Cirrus and extragalactic sources may contaminate the MIPS photometry and create the appearance of an infrared excess. Since we expect the emission from a circumstellar disk to be point-like and centered on the star at the distance of Upper Sco, contaminated $24 \mu \mathrm{m}$ photometry can be identified from emission that is extended or offset from the stellar position.

The maximum astrometric offset between the Two Micron All Sky Survey (2MASS) and Spitzer $24 \mu \mathrm{m}$ positions is 1".6 for HIP 78820. The dispersion in the offsets is the same ( $\sim 0.4$ to 0.55 ) for stars with and without infrared excesses (see Section 3.1), indicating that any contamination is not systematically producing offsets in the measured coordinates. This empir- 
Table 1

MIPS 24 and $70 \mu \mathrm{m}$ Photometery

\begin{tabular}{|c|c|c|c|c|c|c|}
\hline \multirow[t]{2}{*}{ Source $^{\mathrm{a}}$} & \multicolumn{2}{|c|}{ MIPS $24 \mu \mathrm{m}^{\mathrm{b}}$} & \multicolumn{2}{|c|}{ MIPS $70 \mu \mathrm{m}^{\mathrm{c}}$} & \multirow[t]{2}{*}{ AORKEY } & \multirow[t]{2}{*}{ Flags } \\
\hline & $S_{v}(\mathrm{mJy})$ & $\sigma(\mathrm{mJy})$ & $S_{v}(\mathrm{mJy})$ & $\sigma(\mathrm{mJy})$ & & \\
\hline HD 142987 & 7.89 & 0.07 & 5.2 & 11.5 & 13908224 & \\
\hline HD 147810 & 9.67 & 0.09 & -8.7 & 10.2 & 13906432 & \\
\hline HD 149598 & 5.56 & 0.05 & 11.0 & 21.6 & 13909504 & \\
\hline HIP 76071 & 10.79 & 0.10 & -11.4 & 8.8 & 13902848 & \\
\hline HIP 76310 & 165.40 & 1.50 & 373.1 & 11.3 & 13904896 & \\
\hline HIP 76633 & 9.26 & 0.08 & 12.0 & 9.0 & 13907968 & \\
\hline HIP 77457 & 8.63 & 0.08 & 14.7 & 12.2 & 13906688 & \\
\hline HIP 77545 & 9.48 & 0.09 & 59.3 & 58.5 & 13899264 & \\
\hline HIP 77635 & 79.15 & 0.71 & -18.1 & 28.3 & 13899264 & \\
\hline HIP 77815 & 10.85 & 0.10 & 0.0 & 12.7 & 13901568 & \\
\hline HIP 77840 & 68.72 & 0.62 & -58.5 & 25.9 & 13899264 & 1 \\
\hline HIP 77858 & 46.93 & 0.42 & -5.1 & 23.5 & 13899776 & 3 \\
\hline HIP 77859 & 91.89 & 0.83 & -18.4 & 15.2 & 13899776 & \\
\hline HIP 77900 & 19.55 & 0.18 & -12.5 & 10.2 & 13901056 & \\
\hline HIP 77909 & 25.76 & 0.23 & 4.2 & 15.9 & 13899264 & \\
\hline HIP 77911 & 142.60 & 1.30 & 253.1 & 10.5 & 13901568 & \\
\hline HIP 77960 & 8.49 & 0.08 & -15.6 & 9.5 & 13901568 & \\
\hline HIP 78099 & 12.98 & 0.12 & -21.8 & 10.0 & 13901568 & \\
\hline HIP 78104 & 105.50 & 0.90 & 0.5 & 9.7 & 13898752 & \\
\hline HIP 78168 & 34.18 & 0.31 & 20.4 & 13.4 & 13900288 & 5 \\
\hline HIP 78196 & 10.05 & 0.09 & -1.4 & 7.3 & 13903616 & \\
\hline HIP 78207 & 766.40 & 7.30 & 233.3 & 25.4 & 13899008 & \\
\hline HIP 78233 & 6.22 & 0.06 & -2.0 & 8.8 & 13900288 & \\
\hline HIP 78246 & 35.33 & 0.32 & 15.6 & 11.9 & 13900032 & \\
\hline HIP 78265 & 277.90 & 3.10 & 5.2 & 88.2 & 13897472 & 1 \\
\hline HIP 78483 & 8.04 & 0.07 & 7.1 & 15.7 & 13897472 & \\
\hline HIP 78494 & 11.74 & 0.11 & -13.8 & 11.7 & 13900032 & \\
\hline HIP 78530 & 13.10 & 0.12 & 2.2 & 17.9 & 13902336 & \\
\hline HIP 78549 & 12.20 & 0.11 & -9.6 & 12.9 & 13902336 & \\
\hline HIP 78702 & 11.90 & 0.11 & 2.4 & 9.6 & 13905408 & \\
\hline HIP 78809 & 8.54 & 0.08 & -9.3 & 13.1 & 13900544 & 5 \\
\hline HIP 78820 & 451.70 & 4.10 & 46.5 & 13.4 & 13896960 & \\
\hline HIP 78847 & 10.42 & 0.09 & 5.7 & 10.7 & 13902336 & \\
\hline HIP 78877 & 31.33 & 0.28 & -3.0 & 24.8 & 13900544 & \\
\hline HIP 78933 & 144.80 & 1.30 & -4.0 & 19.4 & 13898240 & \\
\hline HIP 78956 & 14.92 & 0.13 & 1.0 & 10.8 & 13905152 & \\
\hline HIP 78963 & 8.85 & 0.08 & 14.7 & 14.8 & 13907200 & \\
\hline HIP 78968 & 14.72 & 0.13 & -2.2 & 11.7 & 13905152 & 5 \\
\hline HIP 78996 & 35.87 & 0.32 & 9.4 & 10.7 & 13900544 & \\
\hline HIP 79083 & 17.87 & 0.16 & -3.8 & 9.7 & 13898240 & \\
\hline HIP 79097 & 10.20 & 0.09 & -6.0 & 9.9 & 13906176 & \\
\hline HIP 79124 & 12.64 & 0.11 & 0.7 & 15.3 & 13903104 & \\
\hline HIP 79156 & 22.62 & 0.20 & 5.5 & 9.4 & 13897984 & \\
\hline HIP 79250 & 12.02 & 0.11 & -25.2 & 12.0 & 13905920 & 5 \\
\hline HIP 79366 & 9.27 & 0.08 & 2.6 & 13.2 & 13905920 & \\
\hline HIP 79374 & 212.20 & 1.90 & 12.0 & 21.3 & 13897984 & \\
\hline HIP 79392 & 7.28 & 0.07 & 2.4 & 12.5 & 13900800 & \\
\hline HIP 79404 & 64.91 & 0.58 & -48.1 & 21.4 & 13899520 & 1 \\
\hline HIP 79410 & 41.25 & 0.37 & 8.1 & 11.9 & 13897984 & \\
\hline HIP 79439 & 23.86 & 0.21 & 32.8 & 15.4 & 13897984 & \\
\hline HIP 79462 & 15.11 & 0.14 & -3.8 & 10.6 & 13905920 & \\
\hline HIP 79530 & 24.74 & 0.22 & 3.5 & 14.0 & 13900800 & \\
\hline HIP 79606 & 11.48 & 0.10 & -12.0 & 14.3 & 13904128 & \\
\hline HIP 79643 & 9.30 & 0.08 & 0.5 & 10.2 & 13900800 & \\
\hline HIP 79644 & 3.03 & 0.04 & -16.7 & 9.8 & 13901312 & \\
\hline HIP 79733 & 5.09 & 0.05 & -2.0 & 9.9 & 13899520 & \\
\hline HIP 79739 & 11.47 & 0.10 & -17.7 & 22.3 & 13900800 & \\
\hline HIP 79771 & 10.63 & 0.10 & -22.5 & 37.0 & 13904640 & \\
\hline HIP 79785 & 18.61 & 0.17 & -6.7 & 13.7 & 13901312 & \\
\hline HIP 79860 & 5.26 & 0.05 & -0.8 & 9.4 & 13908992 & \\
\hline HIP 79878 & 33.90 & 0.31 & 26.1 & 15.9 & 13903872 & \\
\hline HIP 79897 & 12.40 & 0.11 & 1.7 & 10.6 & 13903360 & 5 \\
\hline HIP 80024 & 48.03 & 0.43 & -13.3 & 100.3 & 13901824 & \\
\hline HIP 80059 & 8.00 & 0.07 & -18.8 & 11.9 & 13901312 & \\
\hline HIP 80088 & 22.62 & 0.20 & 79.2 & 13.8 & 13903360 & \\
\hline
\end{tabular}


Table 1

(Continued)

\begin{tabular}{|c|c|c|c|c|c|c|}
\hline \multirow[t]{2}{*}{ Source $^{\mathrm{a}}$} & \multicolumn{2}{|c|}{ MIPS $24 \mu \mathrm{m}^{\mathrm{b}}$} & \multicolumn{2}{|c|}{ MIPS $70 \mu \mathrm{m}^{\mathrm{c}}$} & \multirow[t]{2}{*}{ AORKEY } & \multirow[t]{2}{*}{$\overline{\text { Flags }}$} \\
\hline & $S_{v}(\mathrm{mJy})$ & $\sigma(\mathrm{mJy})$ & $S_{v}(\mathrm{mJy})$ & $\sigma(\mathrm{mJy})$ & & \\
\hline HIP 80112 & 717.90 & 7.80 & 209.4 & 507.6 & 13896704 & \\
\hline HIP 80130 & 9.04 & 0.08 & 2.3 & 12.3 & 13907456 & \\
\hline HIP 80311 & 4.25 & 0.04 & 4.6 & 23.1 & 13902080 & \\
\hline HIP 80324 & 11.37 & 0.10 & 6.6 & 13.8 & 13902592 & \\
\hline HIP 80338 & 24.91 & 0.25 & 168.2 & 42.9 & 13902080 & 3,4 \\
\hline HIP 80493 & 11.50 & 0.10 & 5.2 & 22.6 & 13904384 & \\
\hline HIP 80896 & 8.09 & 0.07 & 26.4 & 10.2 & 13907712 & \\
\hline HIP 81266 & 267.20 & 2.40 & -8.8 & 49.6 & 13897728 & \\
\hline HIP 82319 & 5.60 & 0.05 & 6.3 & 8.5 & 13908736 & \\
\hline HIP 82397 & 17.90 & 0.16 & -4.5 & 11.9 & 13906944 & \\
\hline [PBB2002] USco J155624.8-222555 & 23.98 & 0.22 & 12.8 & 9.5 & 13914368 & \\
\hline [PBB2002] USco J155625.7-224027 & 0.84 & 0.03 & 2.1 & 12.6 & 13912832 & \\
\hline [PBB2002] USco J155629.5-225657 & 0.82 & 0.03 & 4.5 & 10.8 & 13912832 & \\
\hline [PBB2002] USco J155655.5-225839 & 1.89 & 0.04 & 2.8 & 9.8 & 13901568 & \\
\hline [PBB2002] USco J155706.4-220606 & 7.60 & 0.07 & 14.5 & 9.8 & 13916672 & \\
\hline [PBB2002] USco J155729.9-225843 & 6.10 & 0.06 & 25.3 & 12.5 & 13916416 & \\
\hline [PBB2002] USco J155746.6-222919 & 0.91 & 0.03 & -0.6 & 14.9 & 13912832 & \\
\hline [PBB2002] USco J155829.8-231007 & 24.53 & 0.22 & 6.9 & 10.9 & 13916928 & \\
\hline [PBB2002] USco J155918.4-221042 & 1.17 & 0.04 & 1.5 & 9.2 & 13902336 & \\
\hline [PBB2002] USco J160159.7-195219 & 0.33 & 0.01 & 8.1 & 8.3 & 15728128 & \\
\hline [PBB2002] USco J160210.9-200749 & 0.29 & 0.01 & -6.9 & 9.3 & 13917952 & \\
\hline [PBB2002] USco J160222.4-195653 & 0.69 & 0.02 & 7.3 & 9.7 & 15729664 & \\
\hline [PBB2002] USco J160226.2-200241 & 0.49 & 0.02 & 1.1 & 8.8 & 15729152 & \\
\hline [PBB2002] USco J160245.4-193037 & 0.45 & 0.02 & -0.1 & 10.3 & 13915904 & 5 \\
\hline [PBB2002] USco J160329.4-195503 & 0.54 & 0.02 & -0.1 & 12.3 & 15729152 & 5 \\
\hline [PBB2002] USco J160341.8-200557 & 1.76 & 0.04 & -3.1 & 9.7 & 13905408 & \\
\hline [PBB2002] USco J160343.3-201531 & 1.40 & 0.04 & 5.9 & 12.1 & 13905408 & \\
\hline [PBB2002] USco J160350.4-194121 & 0.68 & 0.03 & -2.5 & 7.9 & 15727872 & \\
\hline [PBB2002] USco J160357.9-194210 & 25.65 & 0.23 & 26.9 & 10.3 & 13912064 & \\
\hline [PBB2002] USco J160418.2-191055 & 0.58 & 0.03 & -15.9 & 12.3 & 15729664 & \\
\hline [PBB2002] USco J160428.4-190441 & 2.31 & 0.04 & -3.2 & 9.1 & 15729920 & \\
\hline [PBB2002] USco J160435.6-194830 & 0.51 & 0.02 & 0.6 & 8.6 & 15728896 & \\
\hline [PBB2002] USco J160439.1-194245 & 0.87 & 0.02 & -2.1 & 9.0 & 15727616 & \\
\hline [PBB2002] USco J160449.9-203835 & 0.59 & 0.02 & -5.2 & 9.0 & 15727360 & \\
\hline [PBB2002] USco J160456.4-194045 & 0.64 & 0.03 & 9.8 & 9.2 & 15729664 & \\
\hline [PBB2002] USco J160502.1-203507 & 1.95 & 0.04 & 4.2 & 11.2 & 13898240 & \\
\hline [PBB2002] USco J160508.3-201531 & 1.79 & 0.04 & -14.1 & 10.8 & 13905408 & \\
\hline [PBB2002] USco J160516.1-193830 & 0.30 & 0.01 & -2.1 & 10.5 & 13917952 & \\
\hline [PBB2002] USco J160517.9-202420 & 2.42 & 0.04 & 4.1 & 9.8 & 13898240 & \\
\hline [PBB2002] USco J160521.9-193602 & 0.98 & 0.04 & 6.0 & 9.2 & 13905408 & \\
\hline [PBB2002] USco J160525.5-203539 & 5.95 & 0.05 & 1.1 & 11.0 & 15727104 & \\
\hline [PBB2002] USco J160528.5-201037 & 1.07 & 0.04 & -4.1 & 9.9 & 13905408 & \\
\hline [PBB2002] USco J160531.3-192623 & 0.35 & 0.01 & 4.6 & 8.9 & 15728128 & \\
\hline [PBB2002] USco J160532.1-193315 & 3.92 & 0.04 & 19.3 & 9.9 & 15726848 & \\
\hline [PBB2002] USco J160545.4-202308 & 26.22 & 0.24 & 31.9 & 11.7 & 13912064 & \\
\hline [PBB2002] USco J160600.6-195711 & 14.28 & 0.13 & 17.3 & 8.5 & 13912064 & \\
\hline [PBB2002] USco J160611.9-193532 & 0.50 & 0.02 & 2.8 & 9.7 & 15729152 & \\
\hline [PBB2002] USco J160619.3-192332 & 0.29 & 0.01 & -15.5 & 9.6 & 15728384 & \\
\hline [PBB2002] USco J160622.8-201124 & 19.63 & 0.18 & 16.3 & 11.9 & 15729152 & \\
\hline [PBB2002] USco J160628.7-200357 & 0.89 & 0.03 & 14.6 & 9.9 & 13912064 & \\
\hline [PBB2002] USco J160643.8-190805 & 12.89 & 0.12 & 12.2 & 8.7 & 15729920 & \\
\hline [PBB2002] USco J160647.5-202232 & 1.30 & 0.04 & -7.6 & 9.7 & 13898240 & \\
\hline [PBB2002] USco J160702.1-201938 & 8.65 & 0.08 & 7.2 & 11.9 & 15728640 & \\
\hline [PBB2002] USco J160704.7-201555 & 0.32 & 0.01 & -2.0 & 10.1 & 15728640 & \\
\hline [PBB2002] USco J160707.7-192715 & 1.60 & 0.04 & -5.2 & 9.1 & 15729920 & \\
\hline [PBB2002] USco J160708.7-192733 & 0.56 & 0.02 & 5.0 & 8.4 & 15728896 & \\
\hline [PBB2002] USco J160719.7-202055 & 0.84 & 0.02 & 0.1 & 10.4 & 15729408 & \\
\hline [PBB2002] USco J160739.4-191747 & 3.27 & 0.04 & 7.7 & 10.3 & 13903104 & \\
\hline [PBB2002] USco J160801.4-202741 & 2.23 & 0.04 & 0.6 & 13.7 & 13898240 & \\
\hline [PBB2002] USco J160801.5-192757 & 1.58 & 0.04 & -4.1 & 9.6 & 15729920 & \\
\hline [PBB2002] USco J160802.4-202233 & 0.77 & 0.03 & 1.7 & 10.6 & 13913088 & \\
\hline [PBB2002] USco J160804.3-194712 & 0.47 & 0.03 & -10.2 & 11.1 & 13913088 & \\
\hline [PBB2002] USco J160815.3-203811 & 0.73 & 0.03 & -16.1 & 10.8 & 15729408 & \\
\hline [PBB2002] USco J160818.4-190059 & 1.05 & 0.04 & 8.3 & 10.0 & 13903104 & \\
\hline
\end{tabular}


Table 1

(Continued)

\begin{tabular}{|c|c|c|c|c|c|c|}
\hline \multirow[t]{2}{*}{ Source $^{\mathrm{a}}$} & \multicolumn{2}{|c|}{ MIPS $24 \mu \mathrm{m}^{\mathrm{b}}$} & \multicolumn{2}{|c|}{ MIPS $70 \mu \mathrm{m}^{\mathrm{c}}$} & \multirow[t]{2}{*}{ AORKEY } & \multirow[t]{2}{*}{ Flags } \\
\hline & $S_{v}(\mathrm{mJy})$ & $\sigma(\mathrm{mJy})$ & $S_{v}(\mathrm{mJy})$ & $\sigma(\mathrm{mJy})$ & & \\
\hline [PBB2002] USco J160823.2-193001 & 76.90 & 0.69 & 99.9 & 10.7 & 13903104 & \\
\hline [PBB2002] USco J160823.5-191131 & 1.34 & 0.04 & 7.2 & 11.9 & 13903104 & \\
\hline [PBB2002] USco J160823.8-193551 & 2.26 & 0.04 & 8.9 & 8.6 & 15729920 & \\
\hline [PBB2002] USco J160825.1-201224 & 1.27 & 0.04 & 3.3 & 11.5 & 13911040 & \\
\hline [PBB2002] USco J160827.5-194904 & 8.91 & 0.08 & 15.8 & 9.7 & 13913088 & \\
\hline [PBB2002] USco J160843.1-190051 & 0.99 & 0.04 & -15.5 & 10.2 & 13903104 & \\
\hline [PBB2002] USco J160854.0-203417 & 1.26 & 0.04 & 9.7 & 14.3 & 13898240 & \\
\hline [PBB2002] USco J160900.0-190836 & 21.17 & 0.19 & 6.1 & 11.8 & 13914880 & 5,6 \\
\hline [PBB2002] USco J160900.7-190852 & 285.80 & 2.60 & 251.3 & 12.5 & 13903104 & \\
\hline [PBB2002] USco J160903.9-193944 & 0.76 & 0.03 & 2.0 & 8.6 & 13912320 & \\
\hline [PBB2002] USco J160904.0-193359 & 0.62 & 0.02 & 0.9 & 9.9 & 13914880 & \\
\hline [PBB2002] USco J160913.4-194328 & 0.66 & 0.03 & -6.6 & 9.9 & 13913088 & \\
\hline [PBB2002] USco J160915.8-193706 & 0.33 & 0.01 & 6.6 & 10.6 & 15728384 & \\
\hline [PBB2002] USco J160933.8-190456 & 1.44 & 0.04 & -22.0 & 13.7 & 13903104 & \\
\hline [PBB2002] USco J160946.4-193735 & 1.55 & 0.04 & 2.3 & 12.8 & 13897984 & \\
\hline [PBB2002] USco J160953.6-175446 & 9.17 & 0.08 & 14.8 & 10.8 & 15726592 & \\
\hline [PBB2002] USco J160954.4-190654 & 2.35 & 0.04 & 22.3 & 15.2 & 13897984 & \\
\hline [PBB2002] USco J160959.4-180009 & 113.60 & 1.00 & 127.6 & 15.2 & 13912576 & \\
\hline [PBB2002] USco J161010.4-194539 & 0.87 & 0.03 & 6.9 & 10.2 & 13912320 & \\
\hline [PBB2002] USco J161011.0-194603 & 0.78 & 0.01 & 2.4 & 12.5 & 13917440 & \\
\hline [PBB2002] USco J161014.7-191909 & 1.68 & 0.04 & 2.9 & 11.0 & 13897984 & \\
\hline [PBB2002] USco J161021.5-194132 & 1.52 & 0.04 & -15.7 & 11.1 & 13897984 & \\
\hline [PBB2002] USco J161024.7-191407 & 0.80 & 0.03 & 7.4 & 12.5 & 13912320 & \\
\hline [PBB2002] USco J161026.4-193950 & 0.85 & 0.03 & -3.5 & 10.2 & 13912320 & \\
\hline [PBB2002] USco J161031.9-191305 & 3.05 & 0.04 & 5.3 & 20.3 & 13897984 & \\
\hline [PBB2002] USco J161052.4-193734 & 1.14 & 0.03 & 9.6 & 11.3 & 13914112 & 1 \\
\hline [PBB2002] USco J161115.3-175721 & 72.82 & 0.66 & 18.1 & 11.8 & 13910784 & \\
\hline [PBB2002] USco J161118.1-175728 & 2.29 & 0.05 & -15.1 & 11.7 & 13910784 & \\
\hline [PBB2002] USco J161420.2-190648 & 1170.00 & 11.00 & 630.1 & 24.8 & 13908480 & 5 \\
\hline PPM 732705 & 3.65 & 0.04 & -6.8 & 11.4 & 13910016 & \\
\hline PPM 747651 & 3.63 & 0.04 & 14.1 & 8.7 & 13910272 & \\
\hline PPM 747978 & 2.90 & 0.06 & 0.9 & 15.7 & 13910528 & \\
\hline [PZ99] J153557.8-232405 & 1.39 & 0.04 & 0.8 & 10.0 & 13911808 & \\
\hline [PZ99] J154413.4-252258 & 2.39 & 0.05 & -12.5 & 13.5 & 13905664 & \\
\hline [PZ99] J155106.6-240218 & 1.52 & 0.07 & -16.9 & 11.9 & 13899776 & \\
\hline [PZ99] J155716.6-252918 & 2.82 & 0.04 & -3.8 & 14.8 & 13897472 & \\
\hline [PZ99] J155750.0-230508 & 1.88 & 0.04 & 7.3 & 10.2 & 13901568 & 5 \\
\hline [PZ99] J155812.7-232835 & 14.42 & 0.13 & 31.1 & 42.5 & 13909248 & \\
\hline [PZ99] J1600.00.7-250941 & 2.71 & 0.04 & 13.9 & 16.0 & 13897472 & \\
\hline [PZ99] J160013.3-241810 & 2.45 & 0.04 & -13.0 & 10.2 & 13900032 & \\
\hline [PZ99] J160031.3-202705 & 3.02 & 0.04 & -1.9 & 9.9 & 13900288 & \\
\hline [PZ99] J160042.8-212737 & 2.60 & 0.04 & 1.8 & 10.4 & 13900288 & \\
\hline [PZ99] J160108.0-211318 & 3.25 & 0.04 & -0.3 & 9.3 & 13900288 & \\
\hline [PZ99] J160147.4-204945 & 4.07 & 0.04 & -3.4 & 11.6 & 13900288 & \\
\hline [PZ99] J160200.3-222123 & 4.56 & 0.04 & -10.4 & 10.7 & 13902336 & \\
\hline [PZ99] J160239.1-254208 & 2.11 & 0.04 & 4.7 & 16.3 & 13897472 & \\
\hline [PZ99] J160251.2-240156 & 2.91 & 0.07 & -12.8 & 14.7 & 13900544 & \\
\hline [PZ99] J160354.9-203137 & 3.69 & 0.17 & -0.6 & 12.1 & 13909760 & 5 \\
\hline [PZ99] J160357.6-203105 & 328.00 & 3.00 & 187.8 & 12.7 & 13909760 & \\
\hline [PZ99] J160421.7-213028 & 167.50 & 1.50 & 1917.4 & 24.8 & 13902336 & \\
\hline [PZ99] J160539.1-215230 & 1.87 & 0.04 & -2.1 & 12.4 & 13902336 & \\
\hline [PZ99] J160612.5-203647 & 2.88 & 0.04 & 0.5 & 10.9 & 13898240 & \\
\hline [PZ99] J160654.4-241610 & 2.86 & 0.04 & 0.9 & 10.2 & 13900544 & \\
\hline [PZ99] J160703.9-191132 & 2.43 & 0.04 & -6.2 & 9.5 & 13903104 & \\
\hline [PZ99] J160831.4-180241 & 2.87 & 0.04 & 8.6 & 14.6 & 13910784 & \\
\hline [PZ99] J160856.7-203346 & 3.35 & 0.04 & -11.7 & 10.6 & 13898240 & \\
\hline [PZ99] J160930.3-210459 & 3.06 & 0.04 & 19.4 & 13.0 & 13911040 & \\
\hline [PZ99] J161302.7-225744 & 3.79 & 0.04 & -7.3 & 10.0 & 13905920 & \\
\hline [PZ99] J161933.9-222828 & 3.63 & 0.04 & -8.7 & 10.7 & 13903360 & \\
\hline RX J1548.0-2908 & 3.11 & 0.04 & -4.2 & 13.3 & 13906688 & \\
\hline RX J1550.0-2312 & 2.91 & 0.04 & 0.9 & 17.7 & 13911296 & \\
\hline RX J1550.9-2534 & 5.02 & 0.04 & 4.8 & 18.7 & 13899264 & \\
\hline RX J1554.0-2920 & 3.17 & 0.04 & 3.6 & 9.5 & 13898752 & \\
\hline RX J1558.1-2405A & 2.26 & 0.04 & -46.3 & 37.6 & 13900032 & \\
\hline RX J1600.7-2343 & 0.95 & 0.04 & -9.6 & 13.1 & 13909248 & \\
\hline
\end{tabular}


Table 1

(Continued)

\begin{tabular}{|c|c|c|c|c|c|c|}
\hline \multirow[t]{2}{*}{ Source $^{\mathrm{a}}$} & \multicolumn{2}{|c|}{ MIPS $24 \mu \mathrm{m}^{\mathrm{b}}$} & \multicolumn{2}{|c|}{ MIPS $70 \mu \mathrm{m}^{\mathrm{c}}$} & \multirow[t]{2}{*}{ AORKEY } & \multirow[t]{2}{*}{ Flags } \\
\hline & $S_{v}(\mathrm{mJy})$ & $\sigma(\mathrm{mJy})$ & $S_{v}(\mathrm{mJy})$ & $\sigma(\mathrm{mJy})$ & & \\
\hline RX J1602.8-2401A & 6.92 & 0.06 & 1.6 & 18.0 & 13900544 & \\
\hline RX J1603.6-2245 & 3.99 & 0.04 & 4.2 & 12.5 & 13902336 & \\
\hline SAO 183706 & 10.87 & 0.10 & -17.8 & 25.0 & 13905664 & \\
\hline ScoPMS 13 & 3.08 & 0.04 & -4.5 & 14.8 & 13899776 & \\
\hline ScoPMS 17 & 43.36 & 0.39 & 59.0 & 14.3 & 13901568 & 2,4 \\
\hline ScoPMS 23 & 5.20 & 0.05 & 27.3 & 12.8 & 13902336 & \\
\hline ScoPMS 28 & 1.49 & 0.04 & 1.9 & 11.0 & 13905408 & \\
\hline ScoPMS 29 & 2.48 & 0.04 & -2.8 & 9.6 & 13905408 & \\
\hline ScoPMS 31 & 334.60 & 3.00 & 296.7 & 11.5 & 13905408 & \\
\hline ScoPMS 32 & 0.98 & 0.04 & -1.1 & 11.2 & 13909760 & \\
\hline ScoPMS 45 & 5.48 & 0.10 & 29.0 & 20.9 & 13910784 & \\
\hline
\end{tabular}

Notes.

a Source names with the prefix [PZ99] and [PBB2002] are from Preibisch \& Zinnecker (1999) and Preibisch et al. (2002), respectively.

${ }^{\mathrm{b}}$ MIPS $24 \mu \mathrm{m}$ photometry measured using a flux calibration factor of $0.0447 \mathrm{MJy} \mathrm{sr}^{-1}$

${ }^{\mathrm{c}}$ MIPS $70 \mu \mathrm{m}$ photometry measured using a flux calibration factor of $702 \mathrm{MJy} \mathrm{sr}^{-1}$.

Flags: (1) $24 \mu \mathrm{m}$ photometry is unreliable due to bright, extended nebulosity; (2) $24 \mu \mathrm{m}$ photometry is unreliable due to nearby star; (3) Source is extended at $24 \mu \mathrm{m}$; (4) $70 \mu \mathrm{m}$ photometry is unreliable due to contaminating source or nebulosity; (5) Contaminating source(s) subtracted before $70 \mu \mathrm{m}$ photometry was measured; (6) $70 \mu \mathrm{m}$ photometry measured on AOR 13903104.

(This table is also available in a machine-readable form in the online journal.)

ical result is consistent with the contamination anticipated from extragalactic source counts (Papovich et al. 2004). For each star in the sample, we computed the expected number of galaxies located within the full-width-at-half-maximum (FWHM) size $\left(6^{\prime \prime}\right)$ of the MIPS $24 \mu \mathrm{m}$ PRF that can produce a 30\% photometric excess at $24 \mu \mathrm{m}$. (As described in Section 3.1, 30\% is approximately the minimum excess detectable with these observations.) We find that $\sim$ one star may be contaminated by an extragalactic source bright enough to produce such an excess.

A more significant level of contamination may arise from cirrus and nearby stars. We used both quantitative techniques and visual inspection of the images to identify such cases. First, we compared PRF photometry to aperture photometry obtained with a 6 pixel $(1$ pixel $=2$ ".55) aperture diameter, which is approximately 2.5 times the FWHM size of the MIPS $24 \mu \mathrm{m}$ PRF. For 38 out of the 220 stars in the sample, the aperture photometry deviated from the PRF photometry by more than $10 \%$. We chose $10 \%$ as the threshold since such deviations would impact the significance the $24 \mu \mathrm{m}$ excesses identified in Section 3.1. Visual inspection of the images revealed that in the vast majority of cases, the difference between the aperture and PRF photometry is most likely due to low signal to noise since the aperture size is much larger than the optimal extraction size for relatively faint sources (Naylor 1998). The photometry was deemed questionable for six stars based on the presence of extended nebulosity (HIP 77840, HIP 78265, HIP 79404, and [PBB2002] USco J161052.4-193734) or because the $24 \mu \mathrm{m}$ emission, while centered on the stellar position, is extended (HIP 77858 and HIP 80338). In addition, for the star ScoPMS 17 , a known contaminating source is resolved in the $16 \mu \mathrm{m}$ images (see Paper I) but is unresolved at $24 \mu \mathrm{m}$. These seven stars are flagged in Table 1.

\subsection{MIPS $70 \mu \mathrm{m}$}

MIPS $70 \mu \mathrm{m}$ observations were obtained in photometry mode with an exposure time of $10 \mathrm{~s}$ and the small field size dither pattern. The number of cycles was fixed at 4 for all stars. MIPS
$70 \mu \mathrm{m}$ images were processed with SSC pipeline version S17 that removes the bias, subtracts a dark image, applies a flat field correction, and linearizes the pixel response. Individual BCD images were mosaicked with the Germanium Reprocessing Tools (GeRT) software package S14.0 version 1.1 developed at the SSC. A $40^{\prime \prime} \times 40^{\prime \prime}$ region centered on the source position was excluded when computing the column and time filtering such that the filtering process is not biased by the presence of a bright source. Filtered images were formed into mosaics with MOPEX (Makovoz \& Marleau 2005).

Aperture photometry was performed on the MIPS $70 \mu \mathrm{m}$ mosaics with a custom version of IDLPHOT using an aperture radius of $16^{\prime \prime}$ (4 pixels on the co-added images). The sky level was computed as the mean pixel value in a sky annulus that extended from $40^{\prime \prime}$ to $60^{\prime \prime}$. The aperture was centered on the expected stellar position computed from the world coordinate system keywords contained in the FITS image headers. No centroiding was performed since the signal-to-noise ratio of most $70 \mu \mathrm{m}$ measurements is less than 3 . Visual inspection of the $70 \mu \mathrm{m}$ mosaics identified 19 images where a point source was located within the outer sky annulus or the aperture radius, but offset from the 2MASS stellar position by more than $4^{\prime \prime}$. Before measuring the aperture photometry, a PRF was fitted to the apparent contaminating source and subtracted from the image using MOPEX.

The MIPS $70 \mu \mathrm{m}$ photometry and internal uncertainties are presented in Table 1. Stars for which the $70 \mu$ m photometry was measured on PRF-subtracted images are marked in the table. The adopted calibration factor is $702.0 \mathrm{MJy} \mathrm{sr}^{-1} /\left(\mathrm{DN} \mathrm{s}^{-1}\right)$ with an uncertainty of $7 \%$ as reported on the SSC MIPS calibration web pages. ${ }^{5}$

Of the 220 stars in the sample, 15 have a $70 \mu \mathrm{m}$ signal-to-noise ratio $\geqslant 3$. Photometry for two of these stars is compromised. ScoPMS 17 is contaminated by a nearby source resolved in the $16 \mu \mathrm{m}$ images (see Paper I). HIP 80338 is surrounded

\footnotetext{
5 http://ssc.spitzer.caltech.edu/mips/calib
} 
Table 2

Normalized Flux Ratios.

\begin{tabular}{|c|c|c|c|c|c|c|c|c|c|}
\hline \multirow[t]{2}{*}{ Source } & \multirow[t]{2}{*}{$\mathrm{SpT}$} & \multirow{2}{*}{$\begin{array}{l}\text { Ref } \\
\text { SpT }\end{array}$} & \multirow[t]{2}{*}{$A_{\mathrm{V}}$} & \multicolumn{3}{|c|}{ Ratio $^{a}$} & \multicolumn{2}{|c|}{ Excess? } & \multirow[t]{2}{*}{ Type } \\
\hline & & & & $24 \mu \mathrm{m}$ & $70 \mu \mathrm{m}$ & $\sigma(70 \mu \mathrm{m})$ & $24 \mu \mathrm{m}$ & $70 \mu \mathrm{m}$ & \\
\hline HD 142361 & G3V & 2 & 0.2 & 1.02 & 8.7 & 13.9 & & & \\
\hline HD 142987 & G4 & 4 & 1.7 & 0.99 & 6.2 & 13.6 & & & \\
\hline HD 146516 & G0IV & 8 & 0.6 & 0.99 & 6.5 & 18.3 & & & \\
\hline HD 147810 & G1 & 4 & 1.0 & 1.00 & -8.3 & 9.8 & & & \\
\hline HD 149598 & G0 & 4 & 0.7 & 1.13 & 18.5 & 36.2 & & & \\
\hline HIP 76071 & B9V & 2 & 0.3 & 0.98 & -9.9 & 7.6 & & & \\
\hline HIP 76310 & $\mathrm{~A} 0 \mathrm{~V}$ & 2 & 0.1 & 17.17 & 361.0 & 37.6 & $\mathrm{Y}$ & $\mathrm{Y}$ & Debris \\
\hline HIP 76633 & B9V & 2 & 0.3 & 1.30 & 12.1 & 9.1 & & & \\
\hline HIP 77457 & A7IV & 1 & 0.1 & 0.96 & 15.8 & 13.3 & & & \\
\hline HIP 77545 & $\mathrm{~A} 2 / 3 \mathrm{~V}$ & 2 & 1.2 & 1.79 & 104.5 & 103.6 & $\mathrm{Y}$ & & Debris \\
\hline HIP 77635 & $\mathrm{~B} 1.5 \mathrm{Vn}$ & 2 & 0.5 & 0.99 & -2.1 & 3.3 & & & \\
\hline HIP 77815 & $\mathrm{~A} 5 \mathrm{~V}$ & 2 & 0.7 & 1.04 & 0.0 & 10.9 & & & \\
\hline HIP 77840 & $\mathrm{~B} 2.5 \mathrm{Vn}$ & 2 & 0.4 & 0.88 & -7.9 & 3.6 & & & \\
\hline HIP 77858 & $\mathrm{~B} 5 \mathrm{~V}$ & 2 & 0.4 & 0.97 & -1.0 & 4.7 & & & \\
\hline HIP 77859 & $\mathrm{~B} 2 \mathrm{~V}$ & 2 & 0.6 & 1.68 & -3.1 & 2.6 & $\mathrm{Y}$ & & $\mathrm{Be}$ \\
\hline HIP 77900 & B7V & 1 & 0.2 & 0.98 & -6.0 & 4.9 & & & \\
\hline HIP 77909 & B8III/IV & 2 & 0.1 & 0.99 & 1.5 & 5.8 & & & \\
\hline HIP 77911 & B9V & 2 & 0.3 & 9.64 & 159.4 & 17.2 & $\mathrm{Y}$ & $\mathrm{Y}$ & Debris \\
\hline HIP 77960 & A4IV/V & 2 & 0.6 & 1.02 & -17.2 & 10.6 & & & \\
\hline HIP 78099 & $\mathrm{~A} 0 \mathrm{~V}$ & 2 & 0.5 & 1.31 & -15.7 & 7.4 & & & \\
\hline HIP 78104 & B2IV/V & 1 & 0.1 & 0.99 & 0.0 & 0.9 & & & \\
\hline HIP 78168 & B3V & 2 & 0.6 & 0.99 & 5.6 & 3.7 & & & \\
\hline HIP 78196 & A0V & 1 & 0.0 & 0.96 & -1.3 & 6.8 & & & \\
\hline HIP 78207 & B8Ia/Iab & 2 & 0.1 & 4.36 & 12.4 & 1.8 & $\mathrm{Y}$ & $\mathrm{Y}$ & $\mathrm{Be}$ \\
\hline HIP 78233 & $\mathrm{~F} 2 / 3 \mathrm{IV} / \mathrm{V}$ & 2 & 0.3 & 0.95 & -2.9 & 13.1 & & & \\
\hline HIP 78246 & $\mathrm{~B} 5 \mathrm{~V}$ & 2 & 0.2 & 0.99 & 4.1 & 3.2 & & & \\
\hline HIP 78265 & $\mathrm{~B} 1 \mathrm{~V}+\mathrm{B} 2 \mathrm{~V}$ & 2 & 0.2 & 1.07 & 0.2 & 3.0 & & & \\
\hline HIP 78483 & G0V & 1 & 0.4 & 0.98 & 8.3 & 18.3 & & & \\
\hline HIP 78494 & $\mathrm{~A} 2 \mathrm{~mA} 7-\mathrm{F} 2$ & 2 & 0.9 & 0.94 & -11.0 & 9.3 & & & \\
\hline HIP 78530 & B9V & 2 & 0.5 & 1.07 & 1.5 & 12.7 & & & \\
\hline HIP 78549 & B9.5V & 2 & 0.4 & 1.03 & -7.3 & 9.9 & & & \\
\hline HIP 78702 & B9V & 2 & 0.7 & 1.26 & 1.8 & 7.6 & & & \\
\hline HIP 78809 & B9V & 2 & 0.4 & 1.02 & -10.2 & 14.4 & & & \\
\hline HIP 78820 & $\mathrm{~B} 0.5 \mathrm{~V}$ & 2 & 0.7 & 1.06 & 0.9 & 0.3 & & & \\
\hline HIP 78847 & $\mathrm{~A} 0 \mathrm{~V}$ & 2 & 0.5 & 1.00 & 5.1 & 9.6 & & & \\
\hline HIP 78877 & B8V & 2 & 0.1 & 1.03 & -0.9 & 7.4 & & & \\
\hline HIP 78933 & $\mathrm{~B} 1 \mathrm{~V}$ & 2 & 0.4 & 0.91 & -0.3 & 1.3 & & & \\
\hline HIP 78956 & B9.5V & 2 & 0.6 & 1.55 & 1.0 & 10.4 & $\mathrm{Y}$ & & Debris \\
\hline HIP 78963 & A9V & 1 & 0.4 & 0.96 & 15.4 & 15.7 & & & \\
\hline HIP 78968 & B9V & 2 & 0.5 & 1.84 & -2.6 & 13.6 & $\mathrm{Y}$ & & Debris \\
\hline HIP 78996 & A9V & 2 & 0.4 & 3.71 & 9.1 & 10.4 & $\mathrm{Y}$ & & Debris \\
\hline HIP 79083 & F3V & 2 & 0.9 & 1.03 & -2.0 & 5.0 & & & \\
\hline HIP 79097 & F3V & 2 & 0.3 & 1.00 & -5.5 & 9.1 & & & \\
\hline HIP 79124 & $\mathrm{~A} 0 \mathrm{~V}$ & 2 & 0.6 & 1.06 & 0.5 & 11.3 & & & \\
\hline HIP 79156 & $\mathrm{~A} 0 \mathrm{~V}$ & 2 & 0.5 & 2.83 & 6.4 & 11.0 & $\mathrm{Y}$ & & Debris \\
\hline HIP 79250 & A3III/IV & 2 & 0.2 & 1.29 & -19.6 & 9.5 & & & \\
\hline HIP 79366 & $\mathrm{~A} 3 \mathrm{~V}$ & 2 & 0.6 & 0.99 & 2.7 & 13.3 & & & \\
\hline HIP 79374 & B2IV & 2 & 0.9 & 0.95 & 0.5 & 0.9 & & & \\
\hline HIP 79392 & A2IV & 2 & 0.5 & 0.96 & 3.0 & 16.0 & & & \\
\hline HIP 79404 & B2V & 2 & 0.3 & 0.96 & -6.9 & 3.1 & & & \\
\hline HIP 79410 & B9V & 2 & 0.6 & 3.77 & 6.9 & 10.2 & $\mathrm{Y}$ & & Debris \\
\hline HIP 79439 & B9V & 2 & 0.6 & 1.99 & 25.5 & 12.2 & $\mathrm{Y}$ & & Debris \\
\hline HIP 79462 & $\mathrm{G} 2 \mathrm{~V}$ & 2 & 0.3 & 1.51 & -3.5 & 9.9 & $\mathrm{Y}$ & & Debris \\
\hline HIP 79530 & B6IV & 2 & 0.4 & 0.97 & 1.3 & 5.3 & & & \\
\hline HIP 79606 & F6 & 2 & 0.9 & 0.96 & -9.8 & 11.7 & & & \\
\hline HIP 79643 & $\mathrm{~F} 2$ & 2 & 0.6 & 1.85 & 1.0 & 19.0 & $\mathrm{Y}$ & & Debris \\
\hline HIP 79644 & F5 & 2 & 0.8 & 0.93 & -51.4 & 30.5 & & & \\
\hline HIP 79733 & A1mA9-F2 & 1 & 1.4 & 1.00 & -3.6 & 18.1 & & & \\
\hline HIP 79739 & B8V & 2 & 1.0 & 0.99 & -14.4 & 18.1 & & & \\
\hline HIP 79771 & B9V & 2 & 1.1 & 0.97 & -19.7 & 32.5 & & & \\
\hline HIP 79785 & B9V & 2 & 0.2 & 1.07 & -3.4 & 6.9 & & & \\
\hline HIP 79860 & $\mathrm{~A} 0 \mathrm{~V}$ & 1 & 0.3 & 1.03 & -1.3 & 16.7 & & & \\
\hline HIP 79878 & $\mathrm{~A} 0 \mathrm{~V}$ & 1 & 0.0 & 3.23 & 23.1 & 14.3 & $\mathrm{Y}$ & & Debris \\
\hline HIP 79897 & B9V & 2 & 0.5 & 1.11 & 1.3 & 8.0 & & & \\
\hline HIP 80024 & B9II/III & 2 & 0.7 & 3.36 & -8.7 & 65.4 & $\mathrm{Y}$ & & Debris \\
\hline
\end{tabular}


Table 2

(Continued)

\begin{tabular}{|c|c|c|c|c|c|c|c|c|c|}
\hline \multirow[t]{2}{*}{ Source } & \multirow[t]{2}{*}{ SpT } & \multirow{2}{*}{$\begin{array}{l}\text { Ref } \\
\text { SpT }\end{array}$} & \multirow[t]{2}{*}{$A_{\mathrm{V}}$} & \multicolumn{3}{|c|}{ Ratio $^{\mathrm{a}}$} & \multicolumn{2}{|c|}{ Excess? } & \multirow[t]{2}{*}{ Type } \\
\hline & & & & $24 \mu \mathrm{m}$ & $70 \mu \mathrm{m}$ & $\sigma(70 \mu \mathrm{m})$ & $24 \mu \mathrm{m}$ & $70 \mu \mathrm{m}$ & \\
\hline HIP 80059 & A7III/IV & 2 & 0.5 & 0.99 & -21.9 & 14.1 & & & \\
\hline HIP 80088 & A9V & 2 & 0.4 & 3.75 & 122.4 & 24.6 & $\mathrm{Y}$ & $\mathrm{Y}$ & Debris \\
\hline HIP 80112 & B1III & 2 & 1.2 & 0.95 & 2.7 & 6.4 & & & \\
\hline HIP 80130 & A9V & 2 & 0.6 & 1.04 & 2.3 & 12.7 & & & \\
\hline HIP 80311 & $\mathrm{~A} 0 \mathrm{~V}$ & 1 & 1.0 & 0.86 & 10.1 & 50.5 & & & \\
\hline HIP 80324 & $\mathrm{~A} 0 \mathrm{~V}+\mathrm{A} 0 \mathrm{~V}$ & 1 & 0.0 & 1.03 & 5.4 & 11.3 & & & \\
\hline HIP 80338 & B8II & 2 & 2.3 & 1.42 & 62.9 & 17.2 & & & \\
\hline HIP 80493 & B9V & 1 & 0.9 & 1.01 & 4.2 & 18.3 & & & \\
\hline HIP 80896 & F3V & 1 & 0.0 & 0.97 & 30.4 & 12.1 & & & \\
\hline HIP 81266 & B0V & 1 & 0.3 & 1.17 & -0.3 & 1.7 & & & \\
\hline HIP 82319 & F3V & 2 & 0.0 & 1.00 & 10.5 & 14.2 & & & \\
\hline HIP 82397 & $\mathrm{~A} 3 \mathrm{~V}$ & 1 & 0.0 & 2.06 & -4.9 & 12.8 & $\mathrm{Y}$ & & Debris \\
\hline [PBB2002] USco J155624.8-222555 & M4 & 7 & 1.7 & 22.18 & 110.8 & 82.8 & $\mathrm{Y}$ & & Primordial \\
\hline [PBB2002] USco J155625.7-224027 & M3 & 7 & 1.0 & 1.06 & 23.1 & 139.6 & & & \\
\hline [PBB2002] USco J155629.5-225657 & M3 & 7 & 0.9 & 1.06 & 50.6 & 123.2 & & & \\
\hline [PBB2002] USco J155655.5-225839 & M0 & 7 & 0.7 & 1.06 & 13.7 & 48.3 & & & \\
\hline [PBB2002] USco J155706.4-220606 & M4 & 7 & 2.0 & 10.36 & 184.4 & 125.3 & $\mathrm{Y}$ & & Primordial \\
\hline [PBB2002] USco J155729.9-225843 & M4 & 7 & 1.4 & 11.21 & 433.6 & 219.2 & $\mathrm{Y}$ & & Primordial \\
\hline [PBB2002] USco J155746.6-222919 & M3 & 7 & 1.2 & 1.21 & -6.2 & 153.2 & & & \\
\hline [PBB2002] USco J155829.8-231007 & M3 & 7 & 1.3 & 28.99 & 76.5 & 120.7 & $\mathrm{Y}$ & & Primordial \\
\hline [PBB2002] USco J155918.4-221042 & M4 & 7 & 1.3 & 1.01 & 12.0 & 73.1 & & & \\
\hline [PBB2002] USco J160159.7-195219 & M5 & 6 & 0.6 & 0.99 & 231.4 & 237.9 & & & \\
\hline [PBB2002] USco J160210.9-200749 & M5 & 6 & 0.8 & 0.84 & -224.6 & 301.4 & & & \\
\hline [PBB2002] USco J160222.4-195653 & M3 & 6 & 1.2 & 1.05 & 99.6 & 132.4 & & & \\
\hline [PBB2002] USco J160226.2-200241 & M5 & 6 & 0.3 & 0.90 & 21.7 & 166.3 & & & \\
\hline [PBB2002] USco J160245.4-193037 & M5 & 6 & 1.1 & 0.97 & -1.9 & 211.8 & & & \\
\hline [PBB2002] USco J160329.4-195503 & M5 & 6 & 0.3 & 1.08 & -2.0 & 213.6 & & & \\
\hline [PBB2002] USco J160341.8-200557 & M2 & 6 & 0.9 & 1.03 & -16.6 & 51.4 & & & \\
\hline [PBB2002] USco J160343.3-201531 & M2 & 6 & 0.9 & 0.99 & 39.7 & 80.6 & & & \\
\hline [PBB2002] USco J160350.4-194121 & M5 & 6 & 0.6 & 1.00 & -34.4 & 108.8 & & & \\
\hline [PBB2002] USco J160357.9-194210 & M2 & 6 & 1.7 & 22.45 & 219.3 & 86.7 & $\mathrm{Y}$ & & Primordial \\
\hline [PBB2002] USco J160418.2-191055 & M4 & 6 & 0.8 & 0.91 & -253.6 & 198.3 & & & \\
\hline [PBB2002] USco J160428.4-190441 & M3 & 6 & 1.0 & 1.00 & -12.9 & 36.9 & & & \\
\hline [PBB2002] USco J160435.6-194830 & M5 & 6 & 0.8 & 1.11 & 10.0 & 157.1 & & & \\
\hline [PBB2002] USco J160439.1-194245 & M4 & 6 & 0.1 & 1.48 & -33.1 & 141.9 & $\mathrm{Y}$ & & Debris \\
\hline [PBB2002] USco J160449.9-203835 & M5 & 6 & 0.7 & 1.07 & -82.4 & 142.1 & & & \\
\hline [PBB2002] USco J160456.4-194045 & M4 & 6 & 0.2 & 1.03 & 141.7 & 134.1 & & & \\
\hline [PBB2002] USco J160502.1-203507 & M2 & 6 & 1.8 & 1.02 & 20.1 & 53.5 & & & \\
\hline [PBB2002] USco J160508.3-201531 & M4 & 6 & 0.4 & 1.05 & -73.6 & 57.0 & & & \\
\hline [PBB2002] USco J160516.1-193830 & M4 & 6 & 0.7 & 0.89 & -65.6 & 325.6 & & & \\
\hline [PBB2002] USco J160517.9-202420 & M3 & 6 & 0.6 & 0.94 & 15.7 & 37.8 & & & \\
\hline [PBB2002] USco J160521.9-193602 & M1 & 6 & 1.2 & 1.03 & 56.8 & 87.5 & & & \\
\hline [PBB2002] USco J160525.5-203539 & M5 & 6 & 0.8 & 10.22 & 17.4 & 176.4 & $\mathrm{Y}$ & & Primordial \\
\hline [PBB2002] USco J160528.5-201037 & M1 & 6 & 1.0 & 1.06 & -35.4 & 86.4 & & & \\
\hline [PBB2002] USco J160531.3-192623 & M5 & 6 & 0.5 & 1.05 & 122.6 & 240.0 & & & \\
\hline [PBB2002] USco J160532.1-193315 & M5 & 6 & 0.6 & 5.93 & 271.7 & 142.3 & $\mathrm{Y}$ & & Primordial \\
\hline [PBB2002] USco J160545.4-202308 & M2 & 6 & 2.2 & 26.32 & 298.4 & 113.8 & $\mathrm{Y}$ & & Primordial \\
\hline [PBB2002] USco J160600.6-195711 & M5 & 6 & 0.6 & 12.67 & 143.4 & 71.6 & $\mathrm{Y}$ & & Primordial \\
\hline [PBB2002] USco J160611.9-193532 & M5 & 6 & 1.5 & 0.97 & 52.2 & 181.1 & & & \\
\hline [PBB2002] USco J160619.3-192332 & M5 & 6 & 0.6 & 0.85 & -496.6 & 312.1 & & & \\
\hline [PBB2002] USco J160622.8-201124 & M5 & 6 & 0.2 & 37.40 & 289.1 & 214.0 & $\mathrm{Y}$ & & Primordial \\
\hline [PBB2002] USco J160628.7-200357 & M5 & 6 & 0.0 & 1.05 & 153.5 & 105.6 & & & \\
\hline [PBB2002] USco J160643.8-190805 & K6 & 6 & 1.8 & 5.29 & 46.7 & 33.6 & $\mathrm{Y}$ & & Primordial \\
\hline [PBB2002] USco J160647.5-202232 & M2 & 6 & 1.4 & 1.09 & -54.9 & 70.1 & & & \\
\hline [PBB2002] USco J160702.1-201938 & M5 & 6 & 1.0 & 14.53 & 112.6 & 187.0 & $\mathrm{Y}$ & & Primordial \\
\hline [PBB2002] USco J160704.7-201555 & M4 & 6 & 0.8 & 0.96 & -59.3 & 296.9 & & & \\
\hline [PBB2002] USco J160707.7-192715 & M2 & 6 & 1.8 & 1.18 & -30.0 & 53.2 & & & \\
\hline [PBB2002] USco J160708.7-192733 & M4 & 6 & 1.2 & 1.37 & 113.0 & 190.6 & $\mathrm{Y}$ & & Debris \\
\hline [PBB2002] USco J160719.7-202055 & M3 & 7 & 1.6 & 1.44 & 2.3 & 164.7 & $\mathrm{Y}$ & & Debris \\
\hline [PBB2002] USco J160739.4-191747 & M2 & 6 & 1.4 & 2.46 & 53.8 & 72.2 & $\mathrm{Y}$ & & Debris \\
\hline [PBB2002] USco J160801.4-202741 & K8 & 6 & 1.5 & 1.16 & 2.5 & 57.2 & & & \\
\hline [PBB2002] USco J160801.5-192757 & M4 & 6 & 0.3 & 1.00 & -24.1 & 56.4 & & & \\
\hline [PBB2002] USco J160802.4-202233 & M5 & 6 & 0.7 & 1.02 & 20.4 & 129.0 & & & \\
\hline [PBB2002] USco J160804.3-194712 & M4 & 6 & 0.0 & 0.84 & -200.1 & 218.7 & & & \\
\hline [PBB2002] USco J160815.3-203811 & M3 & 6 & 1.5 & 1.22 & -206.0 & 140.2 & & & \\
\hline
\end{tabular}


Table 2

(Continued)

\begin{tabular}{|c|c|c|c|c|c|c|c|c|c|}
\hline \multirow[t]{2}{*}{ Source } & \multirow[t]{2}{*}{ SpT } & \multirow{2}{*}{$\begin{array}{l}\text { Ref } \\
\text { SpT }\end{array}$} & \multirow[t]{2}{*}{$A_{\mathrm{V}}$} & \multicolumn{3}{|c|}{ Ratio $^{\mathrm{a}}$} & \multicolumn{2}{|c|}{ Excess? } & \multirow[t]{2}{*}{ Type } \\
\hline & & & & $24 \mu \mathrm{m}$ & $70 \mu \mathrm{m}$ & $\sigma(70 \mu \mathrm{m})$ & $24 \mu \mathrm{m}$ & $70 \mu \mathrm{m}$ & \\
\hline [PBB2002] USco J160818.4-190059 & M3 & 7 & 1.3 & 1.09 & 74.1 & 88.9 & & & \\
\hline [PBB2002] USco J160823.2-193001 & K9 & 6 & 1.5 & 33.47 & 405.3 & 59.2 & $\mathrm{Y}$ & $\mathrm{Y}$ & Primordial \\
\hline [PBB2002] USco J160823.5-191131 & M2 & 6 & 1.1 & 1.18 & 49.9 & 83.2 & & & \\
\hline [PBB2002] USco J160823.8-193551 & M1 & 6 & 1.5 & 1.01 & 36.6 & 35.7 & & & \\
\hline [PBB2002] USco J160825.1-201224 & M1 & 6 & 1.4 & 1.10 & 24.4 & 84.4 & & & \\
\hline [PBB2002] USco J160827.5-194904 & M5 & 6 & 1.1 & 10.56 & 174.1 & 108.8 & $\mathrm{Y}$ & & Primordial \\
\hline [PBB2002] USco J160843.1-190051 & M4 & 6 & 1.1 & 1.01 & -146.1 & 96.6 & & & \\
\hline [PBB2002] USco J160854.0-203417 & M4 & 7 & 1.3 & 1.02 & 72.1 & 106.4 & & & \\
\hline [PBB2002] USco J160900.0-190836 & M5 & 7 & 0.7 & 30.44 & 81.9 & 158.3 & $\mathrm{Y}$ & & Primordial \\
\hline [PBB2002] USco J160900.7-190852 & K9 & 6 & 0.8 & 101.78 & 834.3 & 93.0 & $\mathrm{Y}$ & $\mathrm{Y}$ & Primordial \\
\hline [PBB2002] USco J160903.9-193944 & M4 & 6 & 0.7 & 0.88 & 24.4 & 105.9 & & & \\
\hline [PBB2002] USco J160904.0-193359 & M4 & 7 & 1.6 & 1.00 & 13.7 & 149.9 & & & \\
\hline [PBB2002] USco J160913.4-194328 & M3 & 6 & 1.4 & 0.99 & -93.8 & 140.3 & & & \\
\hline [PBB2002] USco J160915.8-193706 & M5 & 6 & 0.3 & 0.97 & 184.7 & 296.9 & & & \\
\hline [PBB2002] USco J160933.8-190456 & M2 & 6 & 1.7 & 1.08 & -142.9 & 89.8 & & & \\
\hline [PBB2002] USco J160946.4-193735 & M1 & 6 & 1.6 & 1.07 & 13.8 & 76.6 & & & \\
\hline [PBB2002] USco J160953.6-175446 & M3 & 7 & 1.9 & 22.76 & 341.5 & 252.8 & $\mathrm{Y}$ & & Primordial \\
\hline [PBB2002] USco J160954.4-190654 & M1 & 6 & 0.9 & 1.52 & 133.9 & 92.1 & $\mathrm{Y}$ & & Debris \\
\hline [PBB2002] USco J160959.4-180009 & M4 & 6 & 0.7 & 96.14 & 1006.3 & 156.3 & $\mathrm{Y}$ & $\mathrm{Y}$ & Primordial \\
\hline [PBB2002] USco J161010.4-194539 & M3 & 6 & 1.0 & 1.09 & 74.4 & 109.7 & & & \\
\hline [PBB2002] USco J161011.0-194603 & M5 & 7 & 0.5 & 2.02 & 58.2 & 302.8 & $\mathrm{Y}$ & & Debris \\
\hline [PBB2002] USco J161014.7-191909 & M3 & 6 & 0.9 & 1.50 & 24.0 & 91.5 & $\mathrm{Y}$ & & Debris \\
\hline [PBB2002] USco J161021.5-194132 & M3 & 7 & 1.6 & 1.12 & -96.2 & 68.7 & & & \\
\hline [PBB2002] USco J161024.7-191407 & M3 & 6 & 1.5 & 0.91 & 86.3 & 145.4 & & & \\
\hline [PBB2002] USco J161026.4-193950 & M4 & 7 & 1.9 & 0.94 & -38.2 & 111.6 & & & \\
\hline [PBB2002] USco J161031.9-191305 & K7 & 6 & 1.1 & 1.04 & 16.3 & 62.2 & & & \\
\hline [PBB2002] USco J161052.4-193734 & M3 & 7 & 2.3 & 1.96 & 78.0 & 92.8 & & & \\
\hline [PBB2002] USco J161115.3-175721 & M1 & 6 & 1.6 & 17.71 & 41.1 & 27.1 & $\mathrm{Y}$ & & Primordial \\
\hline [PBB2002] USco J161118.1-175728 & M4 & 6 & 0.9 & 0.99 & -61.3 & 48.0 & & & \\
\hline [PBB2002] USco J161420.2-190648 & K5 & 7 & 1.8 & 40.07 & 201.1 & 21.6 & $\mathrm{Y}$ & $\mathrm{Y}$ & Primordial \\
\hline PPM 732705 & G6 & 4 & 0.7 & 1.00 & -17.3 & 29.2 & & & \\
\hline PPM 747651 & G3 & 4 & 0.3 & 1.05 & 36.2 & 22.7 & & & \\
\hline PPM 747978 & G3 & 4 & 0.7 & 0.94 & 3.0 & 50.6 & & & \\
\hline [PZ99] J153557.8-232405 & K3: & 5 & 0.7 & 0.90 & 5.5 & 66.9 & & & \\
\hline [PZ99] J154413.4-252258 & M1 & 5 & 0.6 & 1.00 & -49.0 & 52.9 & & & \\
\hline [PZ99] J155106.6-240218 & M2 & 3 & 0.4 & 1.16 & -103.2 & 73.5 & & & \\
\hline [PZ99] J155716.6-252918 & M0 & 3 & 0.0 & 0.94 & -12.6 & 48.9 & & & \\
\hline [PZ99] J155750.0-230508 & M0 & 3 & 0.1 & 0.95 & 36.0 & 50.6 & & & \\
\hline [PZ99] J155812.7-232835 & $\mathrm{G} 2$ & 3 & 0.4 & 2.72 & 54.7 & 75.0 & $\mathrm{Y}$ & & Debris \\
\hline [PZ99] J155847.8-175800 & $\mathrm{K} 3$ & 5 & 1.8 & 1.41 & -46.9 & 32.1 & $\mathrm{Y}$ & & Debris \\
\hline [PZ99] J160000.7-250941 & G0 & 3 & 0.0 & 1.09 & 47.9 & 55.1 & & & \\
\hline [PZ99] J160013.3-241810 & M0 & 3 & 0.6 & 1.50 & -74.1 & 58.8 & $\mathrm{Y}$ & & Debris \\
\hline [PZ99] J160031.3-202705 & M1 & 3 & 1.0 & 0.98 & -5.8 & 30.5 & & & \\
\hline [PZ99] J160042.8-212737 & K7 & 3 & 0.8 & 1.01 & 6.5 & 37.5 & & & \\
\hline [PZ99] J160108.0-211318 & M0 & 3 & 0.0 & 0.99 & -0.9 & 26.6 & & & \\
\hline [PZ99] J160147.4-204945 & M0 & 3 & 0.8 & 1.01 & -7.8 & 26.6 & & & \\
\hline [PZ99] J160200.3-222123 & M1 & 3 & 1.1 & 1.48 & -31.2 & 32.5 & $\mathrm{Y}$ & & Debris \\
\hline [PZ99] J160239.1-254208 & K7 & 5 & 0.0 & 0.96 & 20.9 & 72.2 & & & \\
\hline [PZ99] J160251.2-240156 & $\mathrm{K} 4$ & 3 & 0.6 & 1.22 & -41.0 & 47.1 & & & \\
\hline [PZ99] J160354.9-203137 & M0 & 3 & 0.9 & 0.99 & -1.5 & 30.6 & & & \\
\hline [PZ99] J160357.6-203105 & K5 & 3 & 0.9 & 33.16 & 176.9 & 21.3 & $\mathrm{Y}$ & $\mathrm{Y}$ & Primordial \\
\hline [PZ99] J160421.7-213028 & $\mathrm{K} 2$ & 3 & 1.0 & 58.15 & 6204.3 & 623.8 & $\mathrm{Y}$ & $\mathrm{Y}$ & Primordial \\
\hline [PZ99] J160539.1-215230 & M1 & 3 & 0.8 & 1.17 & -10.4 & 61.7 & & & \\
\hline [PZ99] J160612.5-203647 & K5 & 3 & 1.8 & 0.98 & 1.6 & 35.3 & & & \\
\hline [PZ99] J160654.4-241610 & M3 & 5 & 0.0 & 0.93 & 3.0 & 33.4 & & & \\
\hline [PZ99] J160703.9-191132 & M1 & 3 & 1.1 & 1.05 & -23.7 & 36.4 & & & \\
\hline [PZ99] J160814.7-190833 & $\mathrm{K} 2$ & 5 & 1.6 & 0.97 & 15.9 & 32.9 & & & \\
\hline [PZ99] J160831.4-180241 & M0 & 5 & 0.1 & 1.06 & 28.1 & 47.7 & & & \\
\hline [PZ99] J160856.7-203346 & $\mathrm{K} 5$ & 5 & 1.4 & 0.97 & -32.7 & 29.7 & & & \\
\hline [PZ99] J160930.3-210459 & M0 & 5 & 0.0 & 1.14 & 59.3 & 40.2 & & & \\
\hline [PZ99] J161302.7-225744 & $\mathrm{K} 4$ & 5 & 2.3 & 1.00 & -17.9 & 24.7 & & & \\
\hline [PZ99] J161318.6-221248 & G9 & 5 & 0.9 & 0.98 & -20.7 & 12.2 & & & \\
\hline [PZ99] J161329.3-231106 & $\mathrm{K} 1$ & 5 & 2.0 & 1.03 & 28.8 & 25.4 & & & \\
\hline [PZ99] J161402.1-230101 & G4 & 5 & 2.0 & 1.08 & -50.0 & 33.4 & & & \\
\hline [PZ99] J161411.0-230536 & K0 & 5 & 2.4 & 18.02 & 49.1 & 8.0 & $\mathrm{Y}$ & $\mathrm{Y}$ & Primordial \\
\hline
\end{tabular}


Table 2

(Continued)

\begin{tabular}{|c|c|c|c|c|c|c|c|c|c|}
\hline \multirow[t]{2}{*}{ Source } & \multirow[t]{2}{*}{ SpT } & \multirow{2}{*}{$\begin{array}{l}\text { Ref } \\
\text { SpT }\end{array}$} & \multirow[t]{2}{*}{$A_{\mathrm{V}}$} & \multicolumn{3}{|c|}{ Ratio $^{\mathrm{a}}$} & \multicolumn{2}{|c|}{ Excess? } & \multirow[t]{2}{*}{ Type } \\
\hline & & & & $24 \mu \mathrm{m}$ & $70 \mu \mathrm{m}$ & $\sigma(70 \mu \mathrm{m})$ & $24 \mu \mathrm{m}$ & $70 \mu \mathrm{m}$ & \\
\hline [PZ99] J161459.2-275023 & G5 & 5 & 1.0 & 1.57 & 85.8 & 81.1 & $\mathrm{Y}$ & & Debris \\
\hline [PZ99] J161618.0-233947 & G7 & 5 & 1.1 & 1.11 & -25.8 & 24.6 & & & \\
\hline [PZ99] J161933.9-222828 & K0 & 5 & 1.3 & 1.03 & -22.3 & 27.5 & & & \\
\hline RX J1541.1-2656 & G7 & 5 & 1.6 & 1.03 & -152.6 & 56.6 & & & \\
\hline RX J1548.0-2908 & G9 & 3 & 0.7 & 1.05 & -12.7 & 39.8 & & & \\
\hline RX J1550.0-2312 & M2 & 3 & 0.7 & 0.97 & 3.0 & 56.5 & & & \\
\hline RX J1550.9-2534 & F9 & 3 & 0.2 & 0.91 & 8.8 & 34.7 & & & \\
\hline RX J1554.0-2920 & M0 & 3 & 0.4 & 0.95 & 10.4 & 27.9 & & & \\
\hline RX J1558.1-2405A & K4 & 3 & 0.5 & 0.90 & -190.8 & 156.1 & & & \\
\hline RX J1600.6-2159 & G9 & 5 & 0.5 & 1.11 & 21.1 & 33.6 & & & \\
\hline RX J1600.7-2343 & M2 & 3 & 0.5 & 0.90 & -94.6 & 129.1 & & & \\
\hline RX J1602.8-2401A & K0 & 3 & 2.3 & 0.99 & 2.1 & 24.3 & & & \\
\hline RX J1603.6-2245 & G9 & 3 & 1.2 & 1.00 & 9.8 & 29.1 & & & \\
\hline SAO 183706 & G8e & 4 & 1.7 & 1.04 & -15.3 & 21.5 & & & \\
\hline ScoPMS 13 & $\mathrm{M} 1.5 \mathrm{~V}$ & 8 & 0.2 & 0.95 & -13.7 & 44.9 & & & \\
\hline ScoPMS 17 & M1V & 8 & 0.7 & 14.68 & 12.7 & 3.3 & & & \\
\hline ScoPMS 21 & K1IV & 5 & 0.2 & 0.98 & -21.0 & 67.3 & & & \\
\hline ScoPMS 23 & K5IV & 8 & 0.2 & 0.92 & 48.9 & 23.4 & & & \\
\hline ScoPMS 27 & K2IV & 5 & 0.2 & 0.94 & 12.6 & 19.7 & & & \\
\hline ScoPMS 28 & M1V & 8 & 0.7 & 0.96 & 11.6 & 68.5 & & & \\
\hline ScoPMS 29 & $\mathrm{M} 2 \mathrm{~V}$ & 8 & 0.7 & 1.02 & -10.5 & 36.2 & & & \\
\hline ScoPMS 31 & $\mathrm{M} 0.5 \mathrm{~V}$ & 8 & 0.9 & 48.89 & 404.1 & 43.2 & $\mathrm{Y}$ & $\mathrm{Y}$ & Primordia \\
\hline ScoPMS 32 & M3V & 8 & 0.6 & 0.92 & -10.9 & 106.6 & & & \\
\hline ScoPMS 45 & K5IV & 8 & 0.7 & 1.44 & 71.2 & 51.7 & $\mathrm{Y}$ & & Debris \\
\hline ScoPMS 52 & KOIV & 8 & 1.3 & 0.97 & 35.3 & 24.6 & & & \\
\hline
\end{tabular}

Note. ${ }^{\text {a }}$ Ratio of the observed flux density to the photospheric value. The $24 \mu \mathrm{m}$ ratios have an adopted uncertainty of 0.07 .

References. (1) Houk 1982; (2) Houk \& Smith-Moore 1988; (3) Kunkel 1999; (4) E. Mamajek 2009, private communication; (5) Preibisch et al. 1998; (6) Preibisch et al. 2001; (7) Preibisch et al. 2002; (8) Walter et al. 1994.

(This table is also available in a machine-readable form in the online journal.)

by nebulosity and it is ambiguous if the $70 \mu \mathrm{m}$ emission is associated with the star. In the remainder of this paper, we do not include these two stars in analysis of the $70 \mu \mathrm{m}$ data.

\section{SOURCES WITH INFRARED EXCESSES}

We now combine the $24 \mu \mathrm{m}$ and $70 \mu \mathrm{m}$ photometry for 220 Upper Sco members (i.e., 205 stars from this study, and 15 stars from FEPS; see Section 2) with 2MASS $J$ and $K_{\text {s }}$ (Skrutskie et al. 2006) and IRAC $4.5 \mu \mathrm{m}$ (Paper I; Carpenter et al. 2008) photometry to identify stars with infrared excesses. All photometry was dereddened using the extinction law from Cardelli et al. (1989) assuming $R_{V}=3.1$ for $\lambda \leqslant 2 \mu \mathrm{m}$, the Chapman et al. (2009) reddening law for IRAC and MIPS $24 \mu \mathrm{m}$, and the reddening law compiled by Mathis (1990) for $70 \mu \mathrm{m}$. The visual extinction toward individual stars was obtained from the literature, or derived by us from published spectral types and optical/2MASS photometry following the general procedure described in Carpenter et al. (2008). The visual extinction is less than 2 mag for $97 \%$ of the stars, which implies a correction of less than $4 \%$ when dereddening the $24 \mu \mathrm{m}$ photometry. The adopted spectral types and visual extinction for each star are listed in Table 2.

\subsection{4 um Excesses}

Figure 1 presents the $24 \mu \mathrm{m}$ to $4.5 \mu \mathrm{m}$ flux ratio (三 $\left.R_{24 / 4.5}\right)$ as a function of dereddened $J-K_{\mathrm{s}}$ color for the Upper Sco sample. The $J-K_{\mathrm{s}}$ color should represent the stellar photosphere for most sources since only two sources have an apparent $K_{s}$-band excess (Paper I). We chose to normalize the $24 \mu \mathrm{m}$ photometry to the $4.5 \mu \mathrm{m}$ band as a compromise between reducing the uncertainties in extinction corrections and the range of intrinsic photospheric colors (favoring longer wavelengths), and biasing the results due to weak emission from circumstellar dust (favoring shorter wavelengths). The locus of points with $\log R_{24 / 4.5} \approx-1.4$ has flux ratios roughly consistent with the stellar photosphere for emission in the Rayleigh-Jeans limits. In practice, the value of $R_{24 / 4.5}$ varies systematically with $\left(J-K_{\mathrm{S}}\right)_{\mathrm{o}}$, indicating changes in the photospheric color with spectral type. A number of sources have values of $R_{24 / 4.5}$ that lie substantially above this locus. These sources include the 35 stars from Paper I that have an $8 \mu \mathrm{m}$ and/or $16 \mu \mathrm{m}$ excess as indicated by the black circles and crosses.

We adopted an iterative procedure to determine the locus of photospheric colors in the $R_{24 / 4.5}$ versus $J-K_{\mathrm{S}}$ diagram. The trend between $\log \left(R_{24 / 4.5}\right)$ and $\left(J-K_{\mathrm{s}}\right)_{\mathrm{o}}$ color was fitted using robust linear regression after first excluding the known 35 stars with $8 \mu \mathrm{m}$ or $16 \mu \mathrm{m}$ excesses and the seven stars flagged with suspect $24 \mu \mathrm{m}$ photometry. Sources that deviated from the best-fit line by more than three times the dispersion of the residuals were removed, and the fit was repeated until no additional sources were excluded.

The best-fit relation is shown as the solid line in Figure 1. The dispersion about the best-fit line is $\sigma=0.03$ dex (i.e., $7 \%$ of the photosphere) for the sources included in the fit. The largest deviation below the best-fit line is $-2.5 \sigma$. Given the sample size of 220 stars, we expected only one source more discrepant 


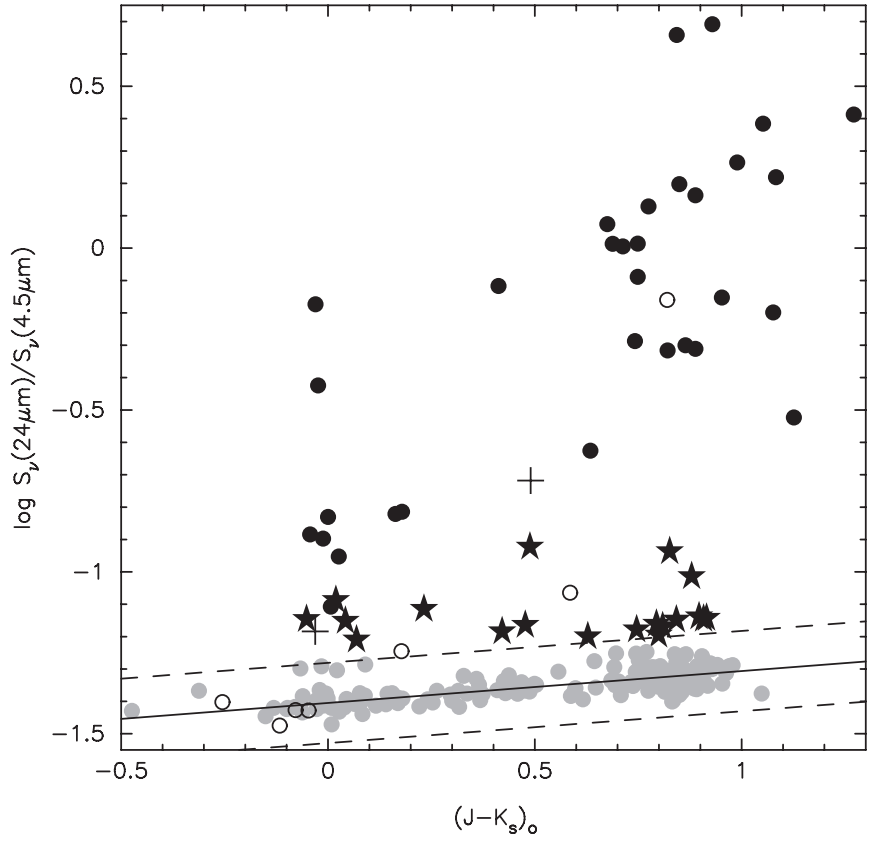

Figure 1. $24 \mu \mathrm{m}$ to $4.5 \mu \mathrm{m}$ flux density ratio $\left(R_{24 / 4.5}\right)$ as a function of dereddened $J-K_{\mathrm{s}}$ color. The solid line indicates the best-fit line (log $R_{24 / 4.5}$ $\left.=0.098\left(J-K_{\mathrm{s}}\right)_{\mathrm{o}}-1.40\right)$ to the main locus of points that is assumed to represent the stellar photosphere. The dashed lines indicate the $\pm 4 \sigma$ limits about the fit $(\sigma=0.03$ dex). Black circles indicate sources with an $8 \mu \mathrm{m}$ or $16 \mu \mathrm{m}$ excess from Paper I. Black stars are sources with detectable excesses at wavelengths $\geqslant 24 \mu \mathrm{m}$, while gray circles indicate sources where the colors are consistent with photospheric emission. Sources with questionable photometry from nebulosity or a contaminating source are indicated by open circles, and known Be stars are marked by the crosses. The source [PBB2002] USco $\mathrm{J} 161420.2-190648$ is offscale on this plot at $\left(J-K_{\mathrm{s}}\right)_{\mathrm{o}}, R_{24 / 4.5}=2.14,0.27$.

than $-2.5 \sigma$ for Gaussian noise. The dispersion of $7 \%$ is larger than the median internal uncertainty $(\sim 2 \%)$, suggesting that either the photometric precision is dominated by uncertainties not been quantified in our data reduction or that many of sources contain weak excesses. We conservatively adopt a $7 \%$ uncertainty on the $R_{24 / 4.5}$ ratio for all sources.

To identify stars that have a $24 \mu \mathrm{m}$ excess, we required that the residuals exceed the photospheric color by $4 \sigma=0.12 \mathrm{dex}$, or a $32 \%$ excess above the photosphere. We adopted $4 \sigma$ as the cutoff since the dispersion in the residuals can vary by $\sim 0.003$ dex depending on which stars are used in the fit. By choosing a $4 \sigma$ cutoff, weak excesses are likely significant even if the dispersion is underestimated by 0.003 dex. In total, 54 stars have a value of $R_{24 / 4.5}$ that exceeds the stellar photosphere by more than $4 \sigma$ and have reliable photometry. Of these 54 stars, 35 have an $8 \mu \mathrm{m}$ and/or $16 \mu \mathrm{m}$ excess, while 19 have a $24 \mu \mathrm{m}$ excess only. These 19 sources are indicated by the filled stars in Figure 1. The sources with excesses only at $24 \mu \mathrm{m}$ span the full range of spectral types and include four A/B-type stars, one F2 star, three G-type stars, two K-type stars, and nine M-type stars.

In addition to the checks described in Section 2.1, we visually re-inspected the $24 \mu \mathrm{m}$ mosaics and the PRF subtracted images for the 54 stars with an infrared excess. Cutouts of the $24 \mu \mathrm{m}$ images and PRF-subtracted images for these sources are presented in Figure 2. We identified seven stars where nebulosity or source confusion may compromise the photometry and create an apparent excess. Three stars (HIP 77545, HIP 80024, and ScoPMS 45) are located in regions of extended nebulosity, although the observed PRF is point-like. Four other stars ([PBB2002] USco J155729.9-225843, [PBB2002] USco
J160532.1-193315, [PBB2002] USco J160708.7-192733, and [PBB2002] USco J160900.0-190836) have a neighboring source within $\sim 20^{\prime \prime}$. We have re-reduced the data for these sources using smaller sky annuli for the PRF fit, and examined various aperture measures. In each case, the infrared excess is robust to these variations in the data reduction. Also, in four of the seven sources, the excess is confirmed by IRAC $8 \mu \mathrm{m}$ or IRS $16 \mu \mathrm{m}$ photometry, where confusion and nebulosity are less of a concern. We therefore classify these seven stars as having an $24 \mu \mathrm{m}$ excess, but we note that the excesses around HIP 77545, [PBB2002] USco J160708.7-192733, and ScoPMS 45 are detected only at $24 \mu \mathrm{m}$ and are otherwise unconfirmed.

The ratio of the observed $24 \mu \mathrm{m}$ flux density to the photospheric value was estimated from the observed photometry and the expected stellar colors. If a source does not have an excess in the $4.5 \mu \mathrm{m}$ or $8 \mu \mathrm{m}$ bands (see Paper I), the observedto-photospheric $24 \mu \mathrm{m}$ ratio was computed from the observed $R_{24 / 4.5}$ ratio and the photospheric value determined from the $R_{24 / 4.5}$ vs. $\left(J-K_{\mathrm{s}}\right)_{\mathrm{o}}$ relation shown in Figure 1. Otherwise, the $24 \mu \mathrm{m}$ photospheric flux density was bootstrapped from the $K_{\mathrm{s}}$-band photometry (or $J$-band if a $K_{\mathrm{s}}$ excess is present) using empirical relations between spectral type and $J-K_{\mathrm{s}}$ color (Paper I; Dahm \& Carpenter 2009), spectral type and $K-M$ color ${ }^{6}$ compiled by Kenyon \& Hartmann (1995), and the $R_{24 / 4.5}$ versus $\left(J-K_{\mathrm{s}}\right)_{\mathrm{o}}$ relation. Table 2 lists the ratio of the observedto-photospheric $24 \mu \mathrm{m}$ flux densities for each star.

\section{2. $70 \mu \mathrm{m}$ Excesses}

The photospheric flux density at $70 \mu \mathrm{m}$ was estimated from the $24 \mu \mathrm{m}$ flux density by assuming that the stellar emission is in the Rayleigh-Jeans limit (i.e., $S_{v} \propto v^{2}$ ). If the source has a known $24 \mu \mathrm{m}$ excess, the photospheric $24 \mu \mathrm{m}$ flux density was estimated as described in Section 3.1. Otherwise, the observed $24 \mu \mathrm{m}$ flux density was used.

Figure 3 presents a histogram of the measured signal-to-noise ratio for the observed $70 \mu \mathrm{m}$ infrared excess above the stellar photosphere. The detected $70 \mu \mathrm{m}$ flux density from the B0.5 star HIP 78820 is consistent with the photospheric emission. Excluding the two stars where the measured $70 \mu \mathrm{m}$ flux density is suspect (see Section 2.2), 12 stars have an apparent $70 \mu \mathrm{m}$ excess greater than $3 \sigma$. Sources with $70 \mu \mathrm{m}$ excesses include four A/B stars, six K stars, and two M stars. All 12 of these stars also have a $24 \mu \mathrm{m}$ excess, as well as an $8 \mu \mathrm{m}$ or $16 \mu \mathrm{m}$ excess identified in Paper I.

\section{NATURE OF THE DISK CANDIDATES}

The circumstellar dust producing the infrared excesses observed in Upper Sco may originate from either a "primordial" or "debris" disk. Conceptually, primordial disks contain copious amounts of gas and dust that are remnants of the star formation process, while debris disks are gas-poor systems created by the collisional shattering of planetesimals.

Distinguishing primordial from debris dust is difficult observationally at an age of 5 Myr. The inner portion of most primordial disks are optically thin by this age as traced by $3-$ $10 \mu \mathrm{m}$ observations (Mamajek et al. 2004; Hernández et al.

\footnotetext{
6 We compared the empirical $K-M$ colors versus the model $K-$ [4.5] colors from the Stellar Performance Estimation Tool (STAR-PET) available on the Spitzer Web site. Between spectral types of A0 and G5, the maximum difference between the two colors is 0.03 mag. For M0 stars, the color difference is $0.15 \mathrm{mag}$. A comparison with the observational data indicates that the $K-M$ colors provide a better match to the data.
} 


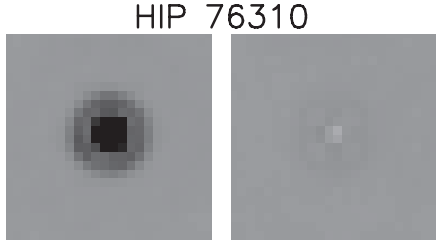

HIP 77859

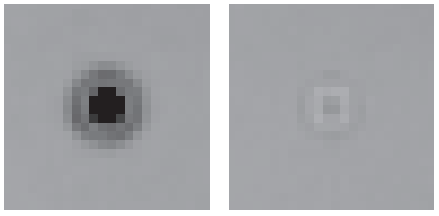

HIP 78207

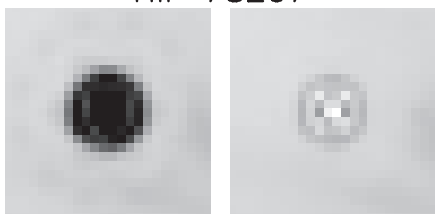

HIP 78968

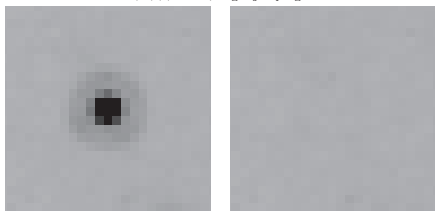

HIP 79156

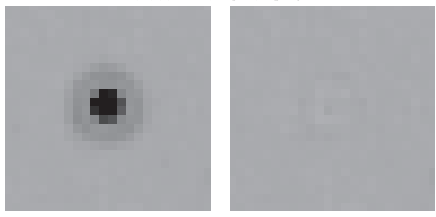

HIP 79439

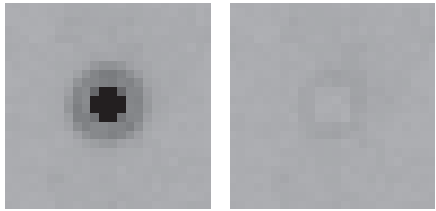

HIP 79643

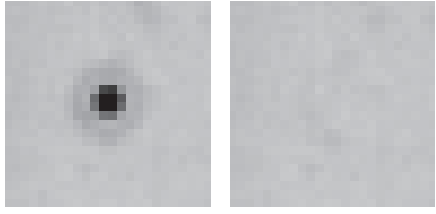

HIP 80024

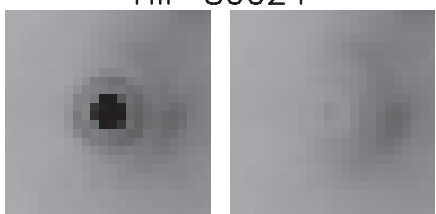

HIP 82397

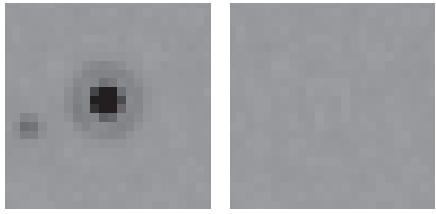

HIP 77545

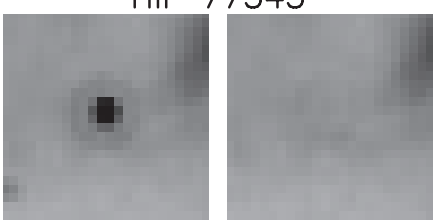

HIP 77911

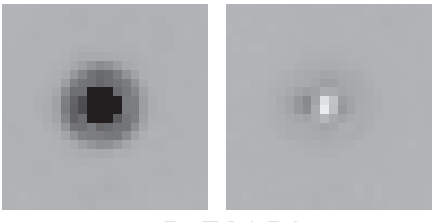

HIP 78956

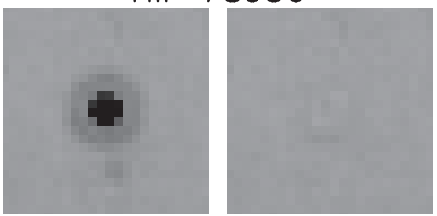

HIP 78996

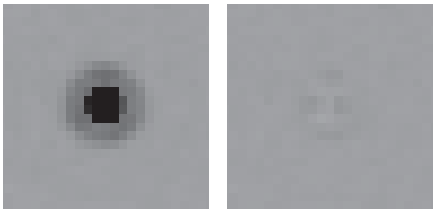

HIP 79410

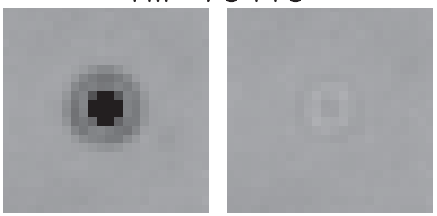

HIP 79462

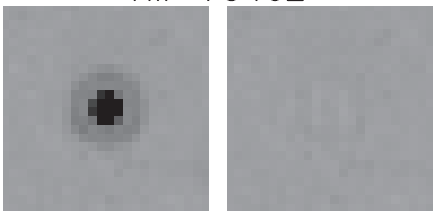

HIP 79878

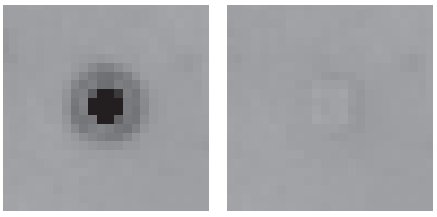

HIP 80088

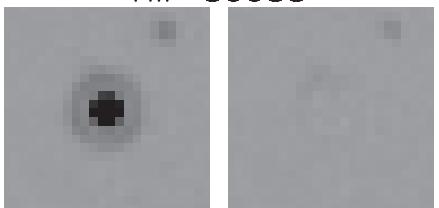

[PBB2002] USco J155624.8-222555
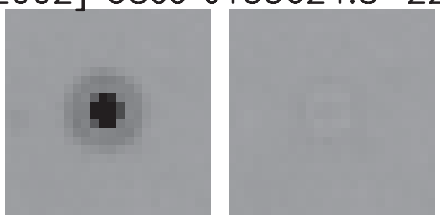

Figure 2. $24 \mu \mathrm{m}$ images for the 54 Upper Sco stars with a $24 \mu \mathrm{m}$ excesses. The left panel for each star shows a $1^{\prime} \times 1^{\prime}$ region centered on the stellar position. The right panel shows the PRF-subtracted image, where all identified stars in the field of view have been fitted and removed. All images are displayed in a logarithmic scale. 


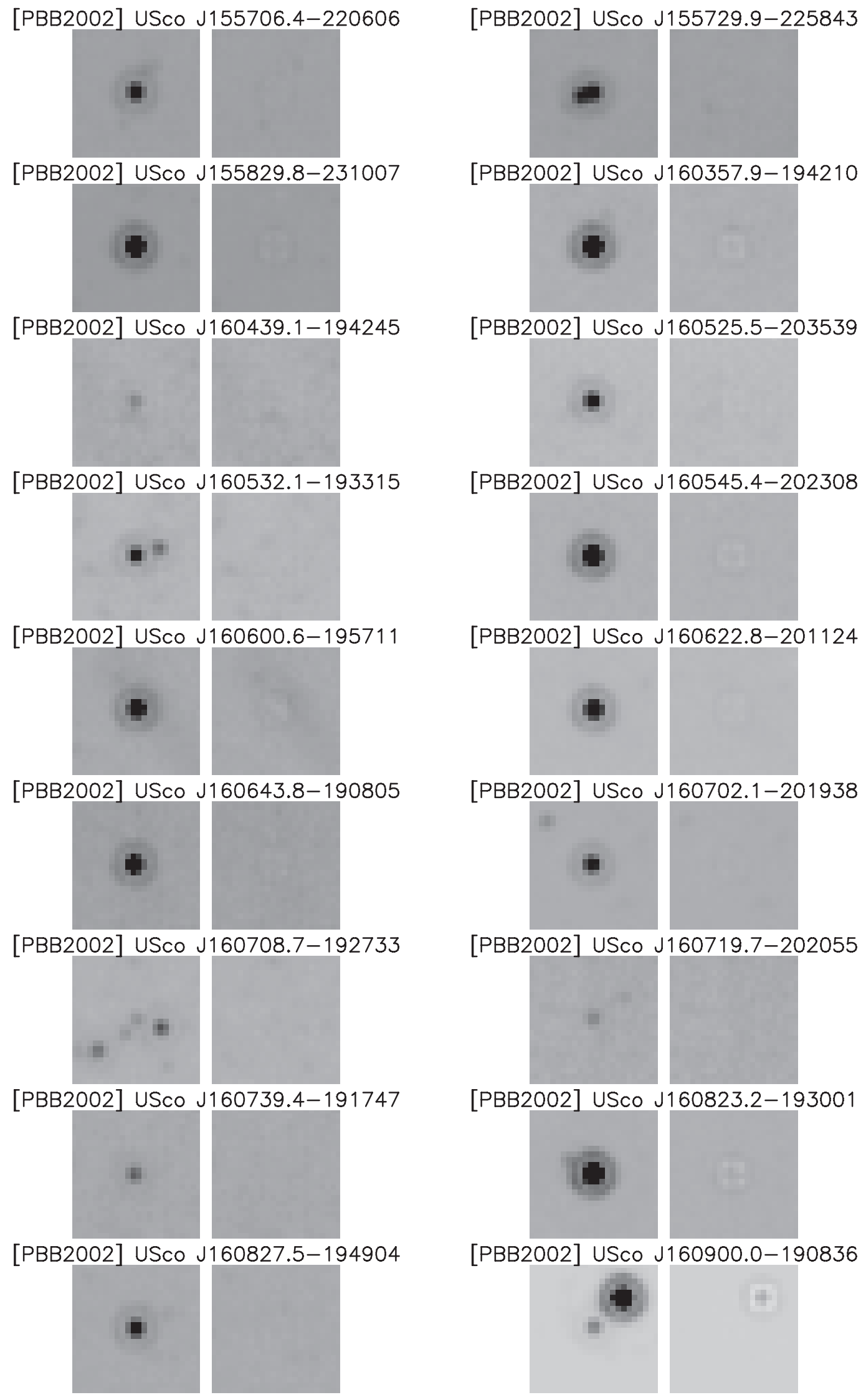

Figure 2. (Continued) 

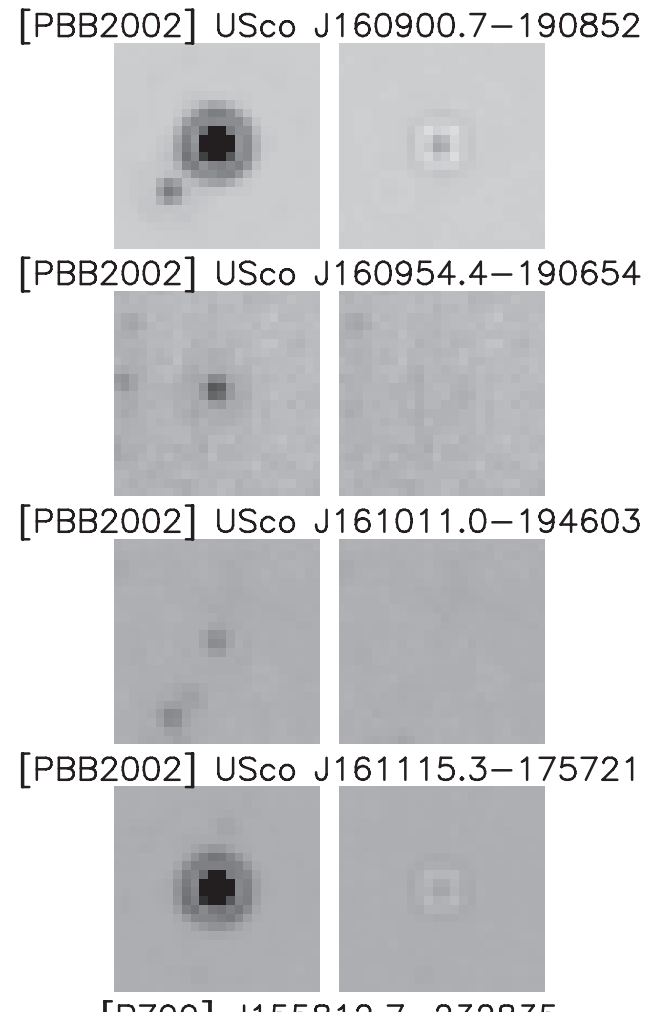

[PZ99] J155812.7-232835
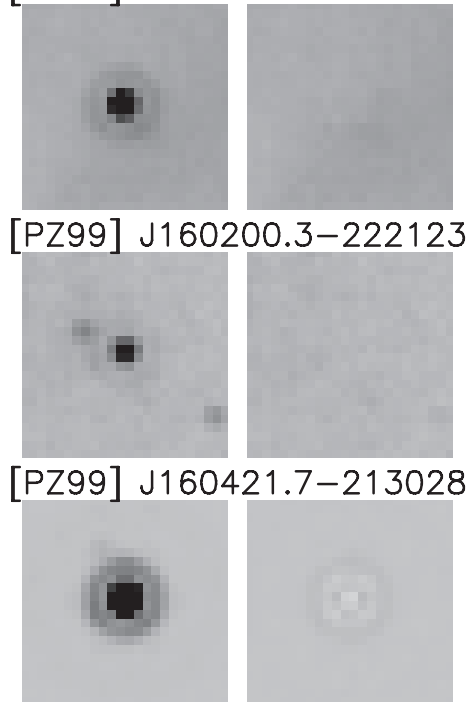

ScoPMS 31

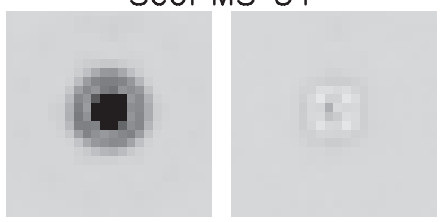

[PBB2002] USco J160953.6-175446

[PBB2002] USco J160959.4-180009

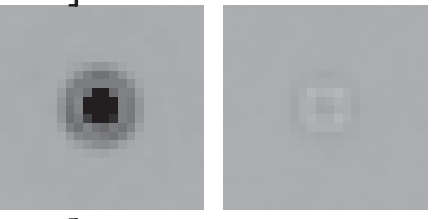

[PBB2002] USco J161014.7-191909

[PBB2002] USco J161420.2-190648

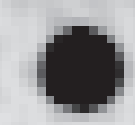

[PZ99] J160013.3-241810

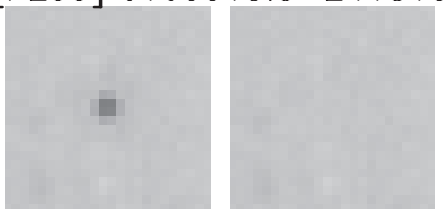

[PZ99] J160357.6-203105

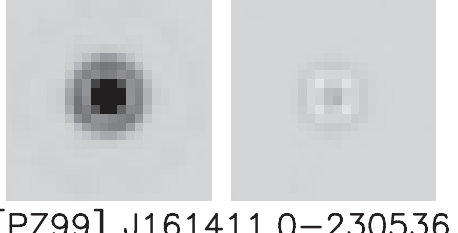

[PZ99] J161411.0-230536

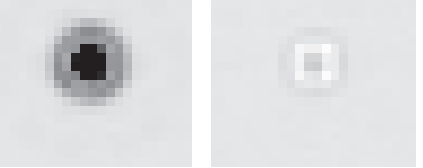

ScoPMS 45

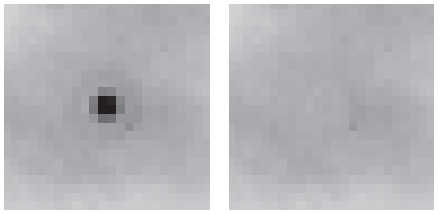

Figure 2. (Continued)

2007), but the state of the outer disk as traced by wavelengths longer than $24 \mu \mathrm{m}$ remain poorly characterized. The classic argument to support the debris interpretation is that radiative and collisional processes can deplete the dust on timescales much shorter than the stellar age assuming the disk is gas poor (Backman \& Paresce 1993). Therefore, the dust must be replenished continuously, presumably from a collisional cascade, to explain the observed infrared excesses. This argument assumes that gas drag is negligible, which can circularize the orbits of the dust grains and reduce the frequency of destructive collisions. Models by Takeuchi \& Artymowicz (2001) indicate that $10 \mathrm{M}_{\oplus}$ of gas distributed radially over $\sim 100 \mathrm{AU}$ can prolong the lifetime of grains around A-type stars to a couple of million years, which is comparable to the age of Upper Sco.

Unfortunately, observations of the gas component are not available for most of the Upper Sco sample. Several stars in Upper Sco have strong $\mathrm{H} \alpha$ emission lines that suggest gas accretion is present, although most stars do not appear to be 


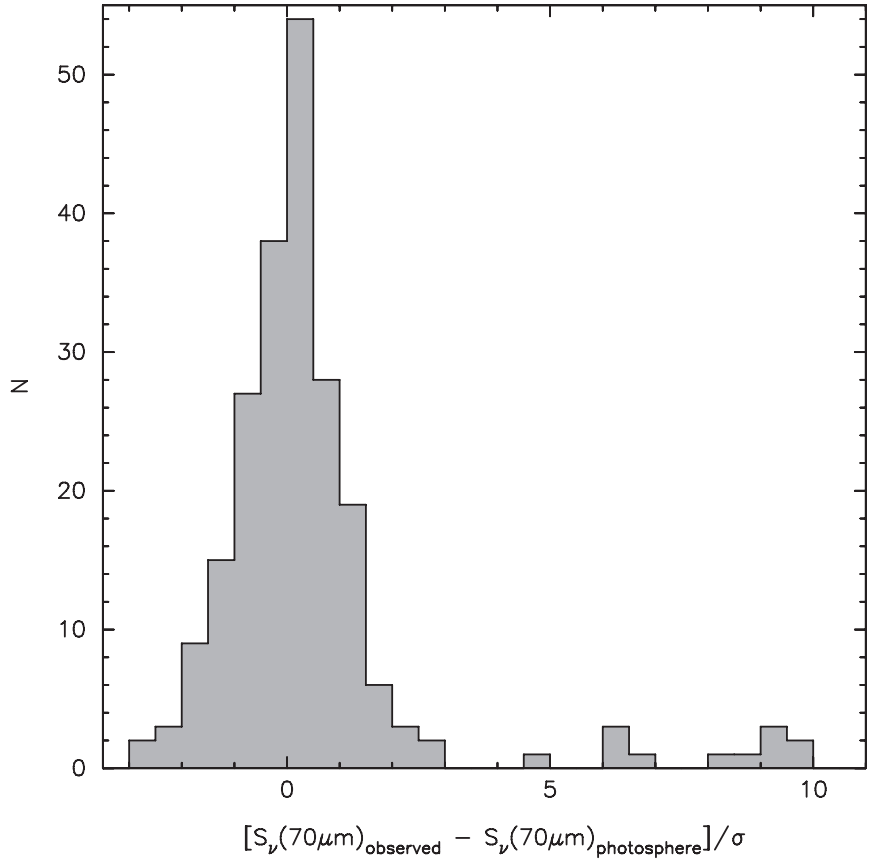

Figure 3. Histogram of the signal-to-noise ratio for the observed $70 \mu \mathrm{m}$ excess above the stellar photosphere. The histogram excludes two stars (ScoPMS 17 and HIP 80338) for which the $70 \mu \mathrm{m}$ photometry is suspect (see Section 2.2). Sources were identified as having a significant excess if the observed $70 \mu \mathrm{m}$ flux density exceeded the photospheric flux density by $\geqslant 3 \sigma$.

accreting detectable levels of material (Dahm \& Carpenter 2009). Yet even these observations only trace gas in the inner disk and are not direct diagnostics of the total gas surface density. Extensive surveys for gas in 5-100 Myr stars have been presented in other studies that are designed to trace the gas over a range of orbital radii (Zuckerman et al. 1995; Najita \& Williams 2005; Pascucci et al. 2006). Even these observations, though, cannot rule out that gas drag has a significant influence on the dust dynamics.

Given the lack of observations of the gas component, we rely on distinctions in the spectral energy distributions (SEDs) between stars in Upper Sco and other known sources to identify potential debris systems. In Paper I, we compared the SEDs of the Upper Sco sample between 2 and $16 \mu \mathrm{m}$ with Herbig $\mathrm{Ae} / \mathrm{Be}$ stars and classical T Tauri stars. We suggested that the infrared excesses around the B/A stars represent debris systems, while the $\mathrm{K} / \mathrm{M}$ stars with $8 \mu \mathrm{m}$ and $16 \mu \mathrm{m}$ excesses originate from optically thick disks, albeit with suppressed levels of midinfrared emission relative to a typical classical $\mathrm{T}$ Tauri star in Taurus. The $24 \mu \mathrm{m}$ photometry and IRS spectra for a subset of these sources support these conclusions (Dahm \& Carpenter 2009).

With the discovery of 19 additional disks in Upper Sco from the $24 \mu \mathrm{m}$ photometric survey, we further investigate the range of disk characteristics. Figure 4 plots the $24 \mu \mathrm{m}$ to $4.5 \mu \mathrm{m}$ flux ratio versus the $8 \mu \mathrm{m}$ to $4.5 \mu \mathrm{m}$ flux ratio for stars in Upper Sco with (solid black circles) and without (gray circles) $24 \mu \mathrm{m}$ excesses. Qualitatively, a dichotomy is present in the excess characteristics. One population, present only for stars with spectral types later than $\mathrm{K} 0$, has both $8 \mu \mathrm{m}$ and $24 \mu \mathrm{m}$ excesses, and has colors similar to stars surrounded by optically thick, primordial disks (Paper I; Dahm \& Carpenter 2009).

The second population of $24 \mu \mathrm{m}$ excess sources, present among all spectral types, has weak or no detectable $8 \mu \mathrm{m}$

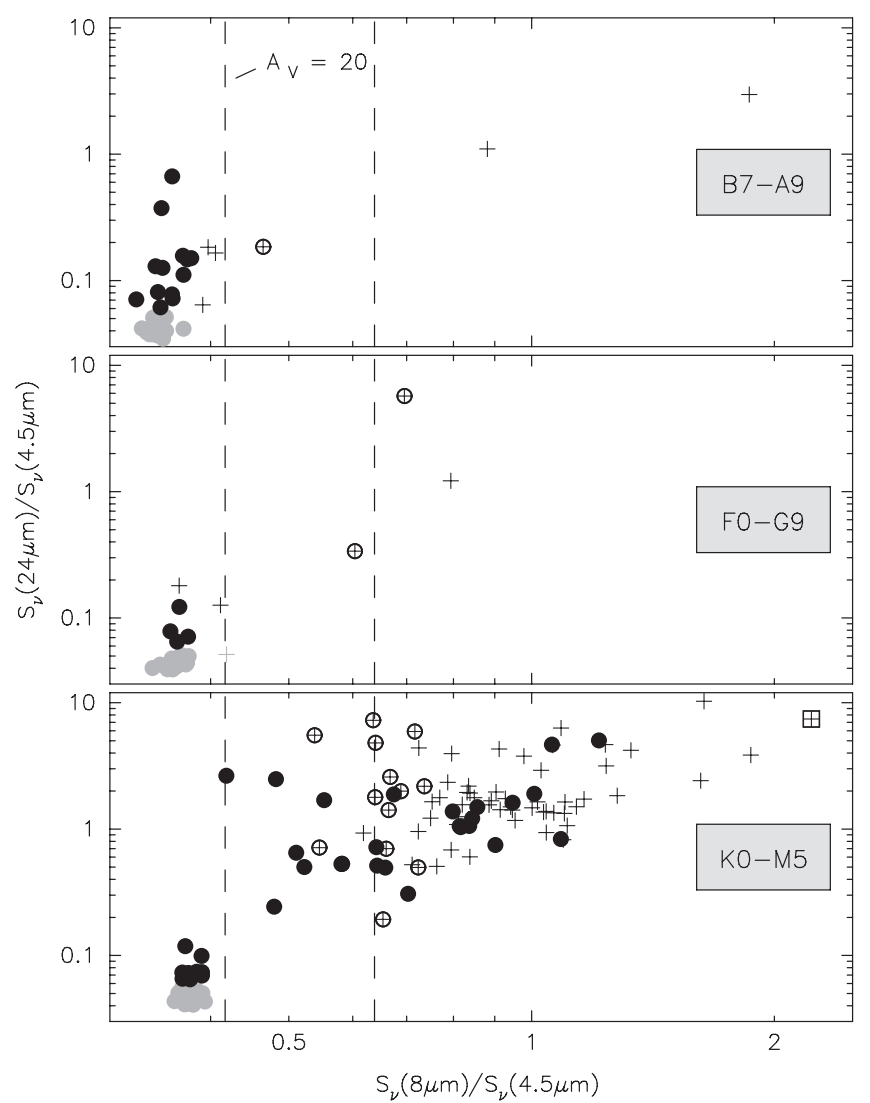

Figure 4. The dereddened $24 \mu \mathrm{m}$ to $4.5 \mu \mathrm{m}$ flux ratio as a function of the dereddened $8 \mu \mathrm{m}$ to $4.5 \mu \mathrm{m}$ flux ratio for B7-A9 stars (top), F0-G9 stars (middle), and K0-M5 stars (bottom). Filled circles represent stars in Upper Sco, and crosses indicate stars in IC 348 that have a $24 \mu \mathrm{m}$ detection (Lada et al. 2006). Black symbols indicate stars with an infrared excess at $24 \mu \mathrm{m}$ (excluding two Be stars), and gray symbols indicate stars that lack a $24 \mu$ m excess. Crosses bounded by circles and squares are stars in IC 348 that have been classified by Lada et al. (2006) as "anemic" or "flat" based on the slope of the SED in the Spitzer IRAC bands. The dashed lines indicate the range of slopes $(\alpha=-2.56$ to 1.80) used to define anemic disks $\left(\alpha=d \log \left(v S_{v}\right) / d \log (v)\right)$. A few anemic disks lie outside these boundaries since the actual slopes were computed using all four IRAC bands. A G0 star in IC 348 is offscale at $(6.4,4.8)$.

excesses. Among the K0-M5 stars, the magnitude of the $24 \mu \mathrm{m}$ excess for this second population is less than the $24 \mu \mathrm{m}$ excess found in any of the sources with both $8 \mu \mathrm{m}$ and $24 \mu \mathrm{m}$ excesses. If we assume the dust is isothermal, a lower limit to the dust temperature obtained from the $24 \mu \mathrm{m}$ excess and the $70 \mu \mathrm{m}$ upper limit is $\sim 53 \mathrm{~K}$ for blackbody emission. The upper limit to the fractional dust luminosity for this dust temperature and a stellar temperature of $4000 \mathrm{~K}$ is $\sim 10^{-4}$. This fractional luminosity is orders of magnitude lower than that found from optically thick disks around classical T Tauri stars.

With the large number of Spitzer surveys of star-forming regions, the primordial disk classification has been refined to include "transitional" (Strom et al. 1989), "pre-transitional" (Espaillat et al. 2007), "evolved" (Hernández et al. 2007), "homologously depleted" (Currie et al. 2009), and "anemic" (Lada et al. 2006) designations. Each of these disk types is characterized by reduced levels of emission at wavelengths $\lesssim$ $10 \mu \mathrm{m}$ relative to classical $\mathrm{T}$ Tauri stars. The reduced infrared emission can be caused by a number of processes, including dynamical clearing of dust by a companion, grain growth, and disk settling.

To compare the characteristics of the Upper Sco sample with anemic disks, Figure 4 shows the infrared colors from Lada et al. 
(2006; see also Currie \& Kenyon 2009) for confirmed members of IC 348 (see Luhman et al. 2003). IC 348 was chosen for this comparison since the cluster contains a similar range of spectral types as Upper Sco, and was used to define anemic disks. One limitation, however, is that the IC 348 observations detected the photosphere at $24 \mu \mathrm{m}$ for A-type stars only, and are not sensitive to the weak $24 \mu \mathrm{m}$ excesses observed around the later type stars in Upper Sco. The vertical dashed lines show the range of slopes in the IRAC bands used to define anemic disks, while an $8 \mu \mathrm{m}$ to $4.5 \mu \mathrm{m}$ flux ratio greater than 0.64 would be classified as an optically thick disk (Lada et al. 2006). None of the B/A/F/G stars in Upper Sco with an infrared excess would be classified with anemic or optically thick disk based on these criteria. The circled crosses in Figure 4 show the actual stars in IC 348 that were identified by Lada et al. (2006) to have anemic disks based on the fitted slope to all four IRAC bands. For B7-A9 (top panel in Figure 4) and F/G spectral types (middle panel), the anemic and optically thick disks in IC 348 have larger $8 \mu \mathrm{m}$ excesses than any of the sources in Upper Sco. The $24 \mu \mathrm{m}$ excesses also tend to be larger in IC 348, although the distribution overlaps significantly with Upper Sco.

The Upper Sco sources with excesses detectable at $24 \mu \mathrm{m}$ only also do not fit the operating definition of transitional disks. Transitional disks are noted by a lack of continuum excesses at wavelengths $\lesssim 10 \mu \mathrm{m}$, but retain excesses at longer wavelengths comparable in strength to classical T Tauri stars. As shown in Figure 4, the K/M stars in Upper Sco with excesses at $24 \mu \mathrm{m}$ only have systematically lower $24 \mu \mathrm{m}$ excesses than the stars with optically thick disks. This does not necessarily indicate that the dust is debris, as a spectrum of inner hole sizes may exist, and the weak $24 \mu \mathrm{m}$ excesses may simply indicate a larger inner hole is present in the disks around the Upper Sco sample. While none of these systems were detected at $70 \mu \mathrm{m}$, most primordial disks were not detected at $70 \mu \mathrm{m}$ either. Therefore, the $70 \mu \mathrm{m}$ limits for the weaker $24 \mu \mathrm{m}$ excesses are insufficient to rule out a cold, optically thick outer disk.

In summary, the above discussion suggests that Upper Sco contains two populations of excess sources. The late-type stars with strong $8 \mu \mathrm{m}$ and $24 \mu \mathrm{m}$ excesses plausibly have primordial disks based on the presence of accretion signatures in a few stars, and a similarity in the infrared colors of young stars surrounded by optically thick disks (Paper I; Dahm \& Carpenter 2009). Many of these sources have infrared colors similar to "anemic" disks (Lada et al. 2006). The origin of the dust producing the weak $24 \mu \mathrm{m}$ excesses remains uncertain, but these disks are likely not a simple extension of the transitional or anemic disk designations. Without additional observations, we can only assume that these disks are gas poor and the dust originates from a debris disk, but we cannot rule out that the dust is remnant primordial material.

In Table 2, we classify each of the disks candidates as primordial, debris, or Be. A summary of the classifications by spectral type is provided in Table 3 . Primordial disks were assigned to K/M-type stars that have $8 \mu \mathrm{m}$ and $16 \mu \mathrm{m}$ excesses since their infrared excess characteristics are similar to optically thick disks (see also Dahm \& Carpenter 2009, Paper I). Two stars (HIP 77859 and HIP 78207) have prominent hydrogen emissions in optical spectra and have been identified as classical Be stars (Crampton 1968; Jaschek et al. 1964); the observed infrared excess may originate from free-free emission in an ionized gas disk (Porter \& Rivinius 2003). The remaining sources with infrared excesses were classified as debris disks given the relatively weak excesses at 8,16 , or $24 \mu \mathrm{m}$. As noted
Table 3

Disk Types in Upper Sco

\begin{tabular}{lcccc}
\hline \hline \multirow{2}{*}{ Spt } & $N$ (stars $)$ & \multicolumn{3}{c}{$N($ disks $)$} \\
\cline { 3 - 5 } & & Be & Debris & Primordial \\
\hline B & 37 & 2 & 6 & 0 \\
$\mathrm{~A}$ & 25 & 0 & 7 & 0 \\
$\mathrm{~F}$ & 9 & 0 & 1 & 0 \\
$\mathrm{G}$ & 21 & 0 & 3 & 0 \\
$\mathrm{~K}$ & 27 & 0 & 2 & 7 \\
M & 101 & 0 & 9 & 17 \\
Total & 220 & 2 & 28 & 24 \\
\hline
\end{tabular}

in Paper I, optically thick primordial disks are present in $19 \%$ of the $\mathrm{K} / \mathrm{M}$ stars in the Upper Sco sample, and in none of the B, A, F, and G stars. Debris disks are detected around all spectral types, with percentages of $9 \%$ for $\mathrm{K} / \mathrm{M}$ stars, $13 \%$ of $\mathrm{F} / \mathrm{G}$ stars, and $28 \%$ of $\mathrm{B} / \mathrm{A}$ stars.

\section{PROPERTIES OF THE DEBRIS DISKS}

A direct comparison of the debris fractions between early- and late-type stars does not necessarily inform how the frequency of disks varies with spectral type. Since observations at a given wavelength probe dust at smaller orbital radii around cooler stars for a fixed grain size, the low $24 \mu \mathrm{m}$ debris fraction around $\mathrm{K} / \mathrm{M}$ stars relative to A-stars may simply reflect that the debris is located at large orbital radii and is too cool to detect in the $24 \mu \mathrm{m}$ band.

Nominally, the orbital radius of the debris can be inferred from the dust temperature. This is possible only for the debris disks around the $\mathrm{B} / \mathrm{A}$ stars where the debris emission is detected at two or more wavelengths. For the F/G/K/M stars, the debris emission is detected only at $24 \mu \mathrm{m}$, and a broad range of temperatures and radii can fit the single photometric point. We instead pose the question: can the debris properties inferred around the B/A stars also explain the debris emission observed around the later spectral types?

The emitted radiation from a debris disk varies with spectral type due to differences in stellar heating and radiative "blowout" of small grains (Plavchan et al. 2009). To compare the debris properties between spectral types in a self-consistent manner, we assume the debris is located in a narrow ring at an orbital radius $R$. The particles follow a power-law size distribution, $n(a)$, between grain radii $a_{\min }$ and $a_{\max }$ such that

$$
n(a)=N_{\mathrm{o}}\left(\frac{a}{a_{\min }}\right)^{\alpha},
$$

where $N_{\mathrm{o}}$ is the normalization constant that effectively determines the total grain surface area. The power-law exponent is fixed at $\alpha=-3.5$ as appropriate for an infinite collisional cascade without binary conditions on the minimum and maximum particle size (Dohnanyi 1969). In practice, the maximum grain size was fixed at $a_{\max }=1000 \mu \mathrm{m}$, and the minimum grain size $\left(a_{\min }\right)$ was set to either the blowout size or $0.05 \mu \mathrm{m}$, whichever was larger. Excluding smaller grains does not impact the results significantly since such small grains are inefficient emitters at $24 \mu \mathrm{m}$.

The grain sizes that will remain gravitationally bound to a star were assessed by balancing the stellar gravitational force $\left(F_{\mathrm{g}}\right)$ against repulsive forces from radiation $\left(F_{\mathrm{pr}}\right)$ and stellar winds $\left(F_{\mathrm{sw}}\right)$. The ratio of these forces $(\beta)$ for a particle of radius $a$ is 
given by

$$
\begin{aligned}
\beta(a) & =\frac{F_{\mathrm{pr}}+F_{\mathrm{sw}}}{F_{\mathrm{g}}} \\
& =\frac{3 L_{*}\left(Q_{\mathrm{rad}}(a)+Q_{\mathrm{sw}}(a) \dot{M}_{\mathrm{sw}} v_{\mathrm{sw}} c / L_{*}\right)}{16 \pi G M_{*} \rho c a},
\end{aligned}
$$

where $L_{*}$ is the stellar luminosity, $\dot{M}_{\mathrm{sw}}$ is the stellar mass loss rate, $v_{\mathrm{sw}}$ is the stellar wind velocity, $Q_{\mathrm{rad}}(a)$ is the particle cross section to radiation in units of the geometric cross section, $Q_{\text {sw }}(a)$ is the analogous cross section for stellar winds, and $\rho$ is the grain density (Burns et al. 1979; Gustafson 1994; Strubbe $\&$ Chiang 2006). Assuming the disk is optically thin, particles for which $\beta<0.5$ remain gravitationally bound to the star, while particles with $\beta>0.5$ are ejected. The stellar wind velocity was set to the escape velocity $\left(v_{\mathrm{sw}}=\sqrt{2 G M_{*} / R_{*}}\right)$. The stellar mass loss rate is uncertain by orders of magnitude for these young ages across all spectral types. The force from stellar winds is negligible for the grain radii considered here unless the mass loss rate is $\gtrsim 1000$ times the solar value (Plavchan et al. 2005; Strubbe \& Chiang 2006). We assume $\dot{M}_{\mathrm{sw}}=2 \times 10^{-14} M_{\odot} \mathrm{yr}^{-1}$, which corresponds to the solar mass loss rate (Wood 2004), and that $Q_{\mathrm{sw}}(a)=1$. The dust emission was computed using optical constants for astronomical silicates (Weingartner \& Draine 2001) and assuming $\rho=2.7 \mathrm{~g} \mathrm{~cm}^{-3}$. Stellar photospheres were approximated as blackbodies.

Given the debris disk model, we can normalize the two free model parameters $\left(R\right.$ and $\left.N_{\mathrm{o}}\right)$ using the nine B/A stars in Upper Sco that have both $16 \mu \mathrm{m}$ and $24 \mu \mathrm{m}$ excesses. The ratio of the $16 \mu \mathrm{m}$ to $24 \mu \mathrm{m}$ excess flux densities determines the orbital radius of the dust, $R$. The ratio varies between 2.8 and 10.0 with a median value of 3.8 , which leads to an orbital radius between 9 AU and 40 AU with a median of 15 AU. Given the orbital radius, the total surface area of dust contained in particles gravitationally bound to the $\operatorname{star}(\beta<0.5)$ is set by the magnitude of the $24 \mu \mathrm{m}$ emission given the inferred orbital radius, which determines $N_{\mathrm{o}}$ in Equation (1). The $24 \mu \mathrm{m}$ excess for all $13 \mathrm{~B} / \mathrm{A}$ stars with debris disks varies between 0.5 and 16 times the photosphere, with a median of $\sim 2$.

To compute the excess emission around stars of other spectral types, stellar properties (mass, luminosity, and temperature) were obtained from the $5 \mathrm{Myr}$ pre-main-sequence isochrone of Siess et al. (2000) for solar metallicity. The orbital radius $(R)$ and the size distribution $\left(N_{\mathrm{o}}\right)$ of the dust were fixed to that inferred around the B/A stars. However, for a given $L_{*}$ and $M_{*}$, only grains with $\beta<0.5$ were included in the calculations of the debris emission. Therefore, even though $N_{\mathrm{o}}$ is fixed, the total dust surface area is larger for later spectral types since the radiation blowout size is smaller. The ratio of the expected dust emission excess to the photosphere was computed in this manner for stars between masses of $0.1 M_{\odot}$ and $7 M_{\odot}$.

Figure 5 presents the results of the calculations for $16 \mu \mathrm{m}$ and $24 \mu \mathrm{m}$ excesses. The shape of the model curves reflects variations in stellar heating and the minimum grain size present in the disk. The dust surface area decreases toward early spectral types and tends to reduce the dust emission, which is compensated by warmer dust temperatures. The $24 \mu \mathrm{m}$ excess relative to the photosphere (hereafter referred to as the relative $24 \mu \mathrm{m}$ excess) peaks at a spectral type of $\sim \mathrm{B} 7$ $\left(J-K_{\mathrm{s}} \approx-0.06\right)$, and declines toward more luminous stars as more small grains are ejected by radiation pressure. Toward later spectral types, the relative $24 \mu \mathrm{m}$ excess at first declines despite the increased surface area since the dust temperature decreases
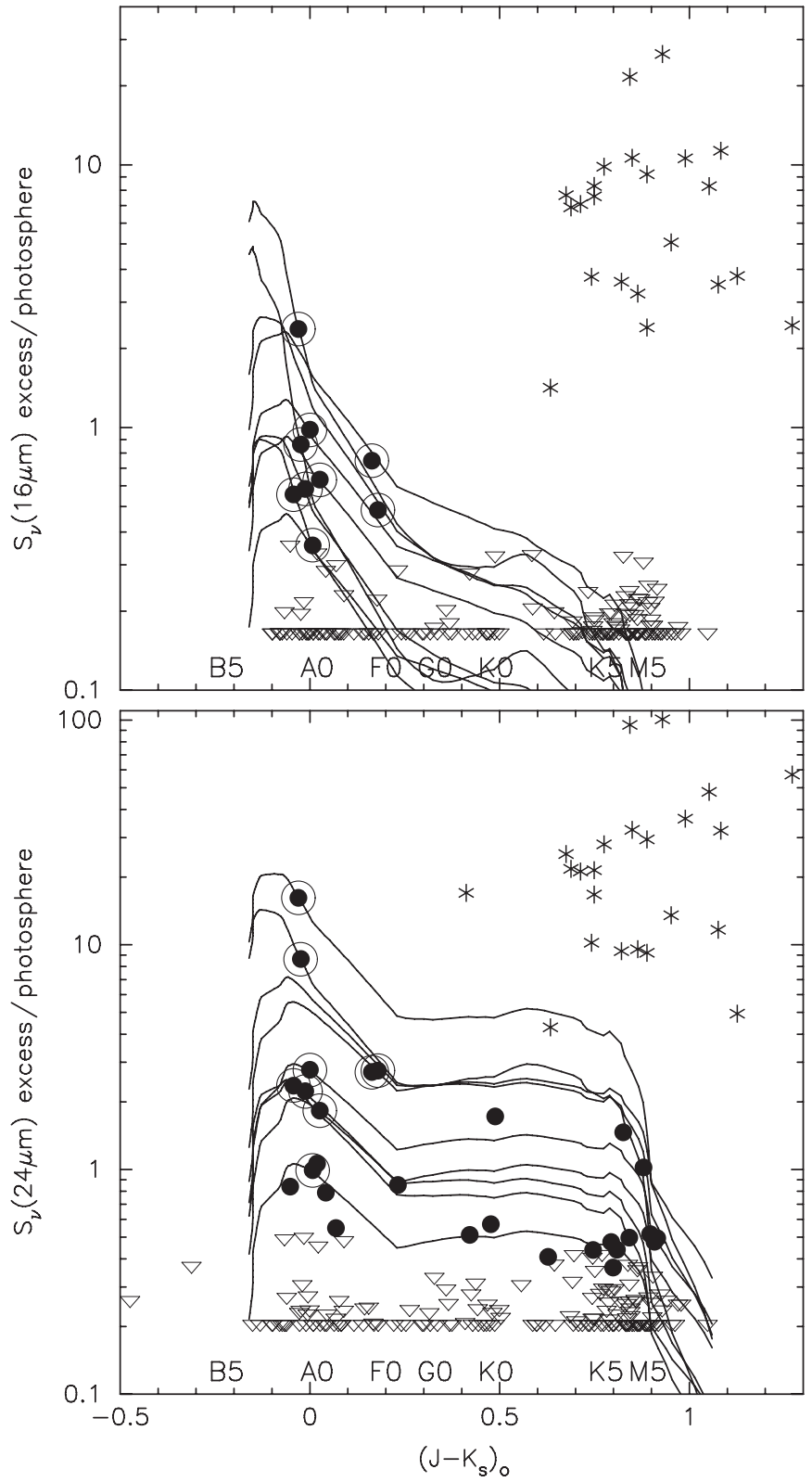

Figure 5. $16 \mu \mathrm{m}$ (top panel) and $24 \mu \mathrm{m}$ (bottom panel) excess in Upper Sco normalized by the photospheric emission as a function of the dereddened $J-K_{\mathrm{s}}$ color. Symbols indicate debris (filled circles) and primordial (asterisks) disks. Triangles indicate the $3 \sigma$ upper limits for stars without detected excesses. Be stars and sources with contaminated photometry have been omitted. The solid curves show the emission from model debris disks with a power-law distribution of particle sizes at a given orbital radius. The models were normalized to the debris properties around B/A stars, and computed around stars of various temperatures after factoring differences in stellar heating and radiation blowout size. Open circles indicate the nine B/A stars used to normalize the models. See text for explanation of model behavior as a function of photospheric color. The results indicate that the magnitude of the $16 \mu \mathrm{m}$ and $24 \mu \mathrm{m}$ excess around the debris disks can be explained by planetesimals belts that have a similar range of orbital radii across all spectral types.

with lower stellar heating. However, at a color of $J-K_{\mathrm{s}} \approx 0.2$ ( $\sim$ F0 star), the blowout particle radius is $\sim 5 \mu \mathrm{m}$, and the dust temperatures are warmer than expected based on blackbody radiation. Combined with the increased surface area of these smaller grains, the normalized $24 \mu \mathrm{m}$ excess ratio plateaus toward cooler stars until a stellar color of $J-K_{\mathrm{s}} \approx 0.8(\sim \mathrm{M} 0$ star). At this point the blowout size is less than $0.1 \mu \mathrm{m}$ and these small grains emit inefficiently at $24 \mu \mathrm{m}$. Therefore the additional 
surface area from small grains does not appreciably increase the amount of infrared excess. The $16 \mu \mathrm{m}$ curves exhibit similar behavior, but fall more steeply toward later spectral types since the dust emission is further into the Wien regime and more sensitive to the dust temperature.

Figure 5 shows that if the only variation in the debris properties is the blowout size and stellar heating, the $24 \mu \mathrm{m}$ excess relative to the photosphere will be $\sim 2$ times higher around $\sim \mathrm{A} 0$ stars compared to F0-M0 stars at an age of $5 \mathrm{Myr}$. The relative $24 \mu \mathrm{m}$ excess decreases rapidly toward stars earlier than B7 and later than M0. Qualitatively, the range of relative $24 \mu \mathrm{m}$ excesses around the $\mathrm{F} / \mathrm{G} / \mathrm{K} / \mathrm{M}$-type stars are consistent with the extrapolation of the excesses around the B/A stars based on this model. At $16 \mu \mathrm{m}$, the relative photometric excess falls sharply with later spectral types and $16 \mu \mathrm{m}$ excesses would be undetectable toward the later type stars. This model is consistent with the lack of $16 \mu \mathrm{m}$ excesses from debris in $\mathrm{F} / \mathrm{G} / \mathrm{K} / \mathrm{M}$ stars (Paper I). We conclude that is not necessary to invoke systematic differences in the radial distribution of dust with spectral type to explain the range of observed $16 \mu \mathrm{m}$ and $24 \mu \mathrm{m}$ excesses.

\section{EVOLUTION OF DEBRIS EMISSION}

Variations in the debris luminosity with stellar age provide a means to investigate the evolution of planetesimal belts and potentially the formation of planetary systems. Dominik \& Decin (2003; see also Wyatt et al. 2007b) suggested that the evolution of debris emission can be explained by quasisteady-state collisional equilibrium of planetesimals distributed in a narrow ring. One limitation of this model is that it does not prescribe a physical mechanism to initiate the collisional cascade. In a series of papers, Kenyon \& Bromley (2008; and references therein) presented a model that follows both the collisional growth of planets and the onset of debris production. They show that the debris disk initially has a low luminosity when planets are still in the formative stages. When the collisional growth produces $\sim 2000 \mathrm{~km}$ size bodies, the resulting gravitational interactions excite planetesimal collisions that leads to dramatically increased debris luminosity. The timescale to form a $2000 \mathrm{~km}$ body increases with the orbital radius, thereby producing a "wave" of debris production in time that propagates from the inner disk outward.

A prediction of the Kenyon \& Bromley model is that the debris luminosity initially increases with time, and then declines once the wave of debris production has propagated outward through the disk on a dynamical timescale. For a debris disk that extends between 30 and $150 \mathrm{AU}$, the debris emission will peak at an age of 5-30 Myr, with faster timescales for higher mass stars given a fixed disk mass. A decline in debris luminosity for ages older than $\sim 20 \mathrm{Myr}$ has been observed in a number of studies and seems firmly established by the data (Habing et al. 2001; Decin et al. 2003; Rieke et al. 2005; Siegler et al. 2007; Carpenter et al. 2009). Hernández et al. (2006) and Currie et al. (2008a) further suggest that the $24 \mu \mathrm{m}$ excess around A/F stars increases from an age of $~ 5 \mathrm{Myr}$ and peaks around 10-15 Myr. If verified, this initial increase in $24 \mu \mathrm{m}$ debris luminosity may signify the onset of planetesimal stirring in debris disks.

The Upper Sco observations presented here provide an important data point to re-evaluate the trends at young ages since few surveys for debris disks are available for younger stars than 10 Myr. As described in the Appendix, we have compiled the results from a number of Spitzer $24 \mu \mathrm{m}$ surveys for comparison to Upper Sco. Similar compilations have appeared in Gaspar et al. (2009), Rebull et al. (2008), Currie et al. (2008a), and Siegler et al. (2007) among others. The compiled surveys include clusters and associations between ages of 2 and $757 \mathrm{Myr}$, and field stars between ages of 3 and 5000 Myr. The young associations and clusters that have been surveyed previously are either sparsely populated with only $\sim 20$ members ( $\eta$ Cha, Gautier et al. 2008; TW Hydra moving group, Low et al. 2005) or are at large distances such that Spitzer is sensitive to the stellar photosphere for only A-type stars or earlier (IC 348, Lada et al. 2006; $\sigma$ Ori, Hernández et al. 2007; Orion OB1a and OB1b, Hernández et al. 2006; NGC 2262, Currie et al. 2008b).

The spectral-type bins were selected to be B7-A9, F0-F9, and G0-K5, which correspond to the spectral-type ranges where numerous Spitzer studies exist (see Appendix). Stars earlier than B7 were omitted since they are fewer in number, and as shown in Section 5 , the debris luminosity may be depressed by radiative blowout of the smallest grains. Stars later than K5 are omitted since most Spitzer surveys of young clusters and associations were insensitive to the stellar photosphere for $\mathrm{K} / \mathrm{M}$ stars. The relationship between the empirical variable of spectral-type and the physical variables stellar mass and luminosity, which dictate debris disk properties, will be discussed in Section 6.3.

As discussed in Section 4, we are faced with the ambiguity of distinguishing if the "anemic," "evolved," and "transitional" disks are debris or primordial systems. We proceed by an entirely observational definition. The disk around HR 4796A is commonly assumed to be debris in nature. The ratio of the observed $24 \mu \mathrm{m}$ emission to the photosphere for this disk is 97, which is one of the largest relative $24 \mu \mathrm{m}$ excesses known. We therefore assume that any B/A/F star with an "anemic," "evolved," or "transitional" classification and has an observedto-photospheric $24 \mu \mathrm{m}$ flux ratio of $\leqslant 100$ is a debris disk, while more luminous systems are primordial disks. For G- and K-type stars, we use a limit of 5 based on the division shown in Figure 4. These divisions are arbitrary and not physically motivated. Changing these boundaries by even a factor of 2 has no substantial impact on our conclusions.

\section{1. $24 \mu m$ Excess Fraction}

We first examine how the fraction of stars with $24 \mu \mathrm{m}$ infrared excesses varies with age. We consider two different thresholds to identify infrared excess: $32 \%$ to incorporate the largest number of studies, and $15 \%$ to increase sensitivity to fainter disks. The adopted thresholds for each survey are listed in the Appendix. Any surveys with sensitivity limits larger than these thresholds were omitted from the appropriate plot. The excess fraction is defined as the ratio of the number of debris disks to the total number of stars (i.e., optically thick disks, debris disks, Be stars, and non-excess stars). Stars were omitted from the statistics if the original study noted that the photometry was contaminated (e.g., nebulosity, nearby bright star) such that it was not possible to determine if the star has an excess or not.

Figure 6 presents the excess fraction for the three spectraltype groupings. For G0-K5 stars, the $24 \mu \mathrm{m}$ excess fraction in Upper Sco is $11 \%(6 / 54)$ at the $32 \%$ detection threshold, which is indistinguishable from the excess fraction (6/56) observed for 10-30 Myr G0-K5 stars. This result is consistent with the FEPS program, which found that the excess fraction for solartype stars is roughly constant for ages $\lesssim 300 \mathrm{Myr}$ (Meyer et al. 2008; Carpenter et al. 2009).

Other studies have found that the debris disk fraction for solartype stars can be as high as $40 \%-45 \%$ between ages of 3 and 30 Myr (Siegler et al. 2007; Gaspar et al. 2009). The difference 


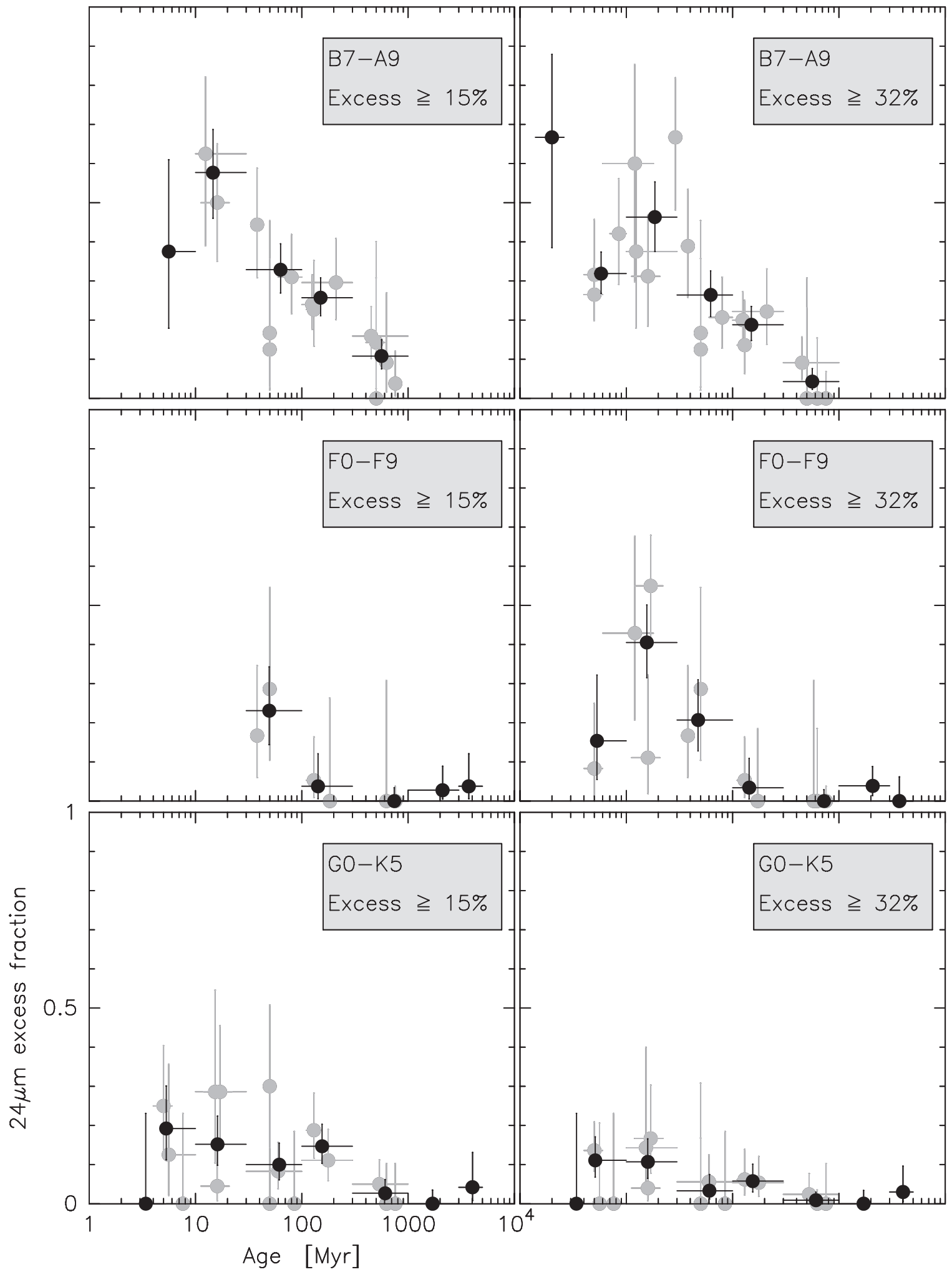

Figure 6. Fraction of sources with a $24 \mu \mathrm{m}$ excess from a debris disk vs. stellar age for the regions summarized in Table 4 . The plots in the left panels show the results for a $24 \mu \mathrm{m}$ excess detection threshold of $15 \%$, and the right panels for $32 \%$. Results are presented for three spectral type ranges. Gray circles represent individual clusters or associations that contain at least five stars in the appropriate spectral type range. Black circles represent the ensemble average of field stars, clusters, and associations regardless of the sample size.

can be attributed to semantics, as these studies have defined "solar-type" stars as FGK-stars. Whether or not F-stars should be included in the definition of solar-type stars is arbitrary. We treat them separately since there is evidence that the excess fraction of F-type stars is higher than G0-K5 stars. In the 10-
30 Myr bin, the excess fraction for F-type stars is 15/37 (41\%) at the $32 \%$ detection threshold, compared to $6 / 56(11 \%)$ for G0-K5 star in the same age bin. The probability that these two observed excess fractions are drawn from the same parent population is $p=0.002$ according to the two-tailed Fisher's exact test. 
For B7-A9 and F0-F9 spectral types, the $24 \mu \mathrm{m}$ excess fraction peaks at $\sim 50 \%$ for ages of $\sim 10-30 \mathrm{Myr}$ and a $32 \%$ detection threshold. The possible peak in the excess fraction result was noted by Currie et al. (2008b), and the decline in the excess fraction toward older ages was found by Rieke et al. (2005). No such increase is apparent for the G/K stars. Gaspar et al. (2009) found that the excess fraction for F-type stars was greater for ages than 3-10 Myr than for 10-30 Myr, which can be attributed to the high excess fraction for F-type stars in Orion OB1b and Orion OB1a (Hernández et al. 2006). Since Hernández et al. (2006) estimate that the Orion $24 \mu \mathrm{m}$ observations are complete for stars earlier than F0 in Orion $\mathrm{OB} 1 \mathrm{a}$ and $\mathrm{A} 8$ in Orion OB1b, the excess fraction for F-stars in Orion may be biased toward higher values and are not included in this analysis.

To evaluate if the excess fraction in the $<10 \mathrm{Myr}$ and 10 $30 \mathrm{Myr}$ age bins could have been drawn from the same parent population for earlier spectral types, we compared the ratios using the two-tailed Fisher's exact test. For a $24 \mu \mathrm{m}$ excess threshold of $32 \%$, which has the largest sample of stars, the probability that these two age bins are drawn from the parent population is $17 \%$ for both B7-A9 and F0-F9 spectral types. If we group B7-F9 spectral types together, the corresponding probability is $9 \%$. The significance of the enhancement in the excess fraction for 10-30 Myr stars relative to $<10 \mathrm{Myr}$ stars then is $\sim 1.7 \sigma$ for stars earlier than F9. Therefore, we conclude that no strong evidence exists for a change in the excess fraction from 2-10 Myr to 10-30 Myr among early-type stars.

\section{2. $24 \mu \mathrm{m}$ Excess}

Evolution in the debris disk properties may manifest itself in the excess luminosity as well as the excess fraction. Figure 7 shows the ratio of the $24 \mu \mathrm{m}$ excess to the photospheric flux as a function of stellar age. Only stars brighter than the photospheric detection limit for the respective samples are shown (see the Appendix). Different symbols are shown for primordial (asterisks) and debris (solid circles) disks; $3 \sigma$ upper limits are shown for stars without a detected excess (open triangles).

For B/A spectral types, the upper envelope of $24 \mu \mathrm{m}$ excess emission declines with time. Rieke et al. (2005) suggested that for ages older than $5 \mathrm{Myr}$, the upper envelope varies with stellar age as $t^{-1}$, which is shown as the dashed line in the top panel of Figure 7 and extrapolated to younger ages. The relative $24 \mu \mathrm{m}$ excesses observed around B/A stars in Upper Sco are consistent qualitatively with this upper envelope. For G0-K5 spectral types, the peak relative $24 \mu \mathrm{m}$ excess is roughly constant for ages less than $300 \mathrm{Myr}$, with a possible decline toward older ages. For F-type stars, a sharp peak in the $24 \mu \mathrm{m}$ excess is present at an age of $\sim 16 \mathrm{Myr}$ as previously noted by Hernández et al. (2006) and Currie et al. (2008a). This peak is due to luminous debris disks in the Lower Centaurus Crux association (Chen et al. 2005).

To evaluate the trends quantitatively, we used the generalized Kendall's Tau test as implemented in the ASURV Rev 1.2 package (Lavalley et al. 1992). This test incorporates both the detections and upper limits to make efficient use of the data. We first consider if evolution is present over all ages in the sample. The probability that a correlation of the $24 \mu \mathrm{m}$ excess versus age is not present for B7-A9, F0-F9, and G0-K5 stars is $3 \times 10^{-10}, 2 \times 10^{-8}$, and $10^{-5}$, respectively. These results confirm the trend apparent from visual inspection of Figure 7 that the magnitude of the $24 \mu \mathrm{m}$ excess decreases with stellar age over all spectral types considered here. This conclusion has been reached previously based on ISO (Habing et al. 2001; Spangler et al. 2001), IRAS (Moór et al. 2006), and Spitzer (Rieke et al. 2005; Su et al. 2006; Siegler et al. 2007; Meyer et al. 2008; Carpenter et al. 2009) observations.

We now consider if evolution in the $24 \mu \mathrm{m}$ excess is present at young ages. We specifically consider the age range between 5 and $17 \mathrm{Myr}$, which is well populated with clusters and associations, and significant evolution is anticipated based on theoretical models for the collisional evolution of a Kuiper Belt analog (Kenyon \& Bromley 2008). For B7-A9, F0-F9, and G0K5 spectral types, the probability that a correlation of the $24 \mu \mathrm{m}$ excess versus age is not present between 5 and $17 \mathrm{Myr}$ is 0.14 , 0.01 , and 0.56 , respectively. Thus when subdivided by spectral type, the only suggestion of a trend in the $24 \mu \mathrm{m}$ excess with age between 5 and $17 \mathrm{Myr}$ is for F-type stars. From inspection of Figure 7, the trend is such that the amount of $24 \mu \mathrm{m}$ excess increases over this age range.

Currie et al. (2008a) reported a significant rise in the magnitude of the debris emission between 5 and $17 \mathrm{Myr}$ based on debris disk observations around $\mathrm{B} / \mathrm{A} / \mathrm{F}$ stars in Orion $\mathrm{OB} 1 \mathrm{~b}$, Orion OB1a, LCC, and UCL. They used the Wilcoxon ranksum test to determine the probability $(p)$ that the mean excess is consistent between two samples. The strongest statistical trend identified by Currie et al. (2008a) was the increase in the mean $24 \mu \mathrm{m}$ excess around $\mathrm{A} / \mathrm{F}$ stars in the $5 \mathrm{Myr}$ Orion OB1b association compared to the 8.5 Myr Orion OB1a association $(p=0.002)$, and Orion OB1b compared to a combined $16-17$ Myr old LCC/UCL sample $(p=0.05)$. One difference in the analysis conducted by Currie et al. (2008a) and this study is that we separated the F-type stars from the B/A stars, and we did not include the F-type stars in Orion OB1a and Orion OB1b. While the separation by spectral type is arbitrary from a physical point of view, the observations are complete for B/A spectral types over a broader range of ages. The MIPS $24 \mu \mathrm{m}$ observations of Orion OB1a and Orion OB1b in particular are complete to the photosphere for F0 and A8 stars (Hernández et al. 2006), respectively, and the $24 \mu \mathrm{m}$ detections of F-type stars in these associations may be biased toward stars with disks.

We stress that we have not included in this analysis the 17 debris disks identified in the MIPS $24 \mu \mathrm{m}$ survey of the $13 \mathrm{Myr}$ old, double cluster h \& $\chi$ Persei (Currie et al. 2008a). These 17 stars have A/F spectral types with relative $24 \mu \mathrm{m}$ excesses that range between 8 and 180 , with a median value of 19 . These excesses are extreme compared to the sources shown in Figure 7. These luminous debris disks could conceivably indicate a peak in the debris production in the 10-20 Myr age range. However, the parent sample for the $\mathrm{h} \& \chi$ Persei is about 30 times larger than the Upper Sco sample. As noted by Currie et al. (2008a), the extreme excesses in $\mathrm{h} \& \chi$ Persei may simply reflect better sampling of the same parent luminosity function. Deeper observations and tabulation of the measured flux densities for all cluster members will determine if the extreme excess sources in $\mathrm{h} \& \chi$ Persei represent a peak in the debris production.

\subsection{Interpretation}

Before drawing further conclusions from the results presented in Section 6.2, we consider how the stellar properties may influence the interpretation of debris disk evolution. The data presented in Figures 6 and 7 were grouped by the observed spectral type. For main-sequence stars, the spectral-type bins of B7-A9, F0-F9, and G0-K5 correspond approximately to stellar masses of 1.9, 1.4, and 0.8 $M_{\odot}$. The corresponding KelvinHelmholtz contraction times are $\sim 3,10$, and $30 \mathrm{Myr}$. Since the 


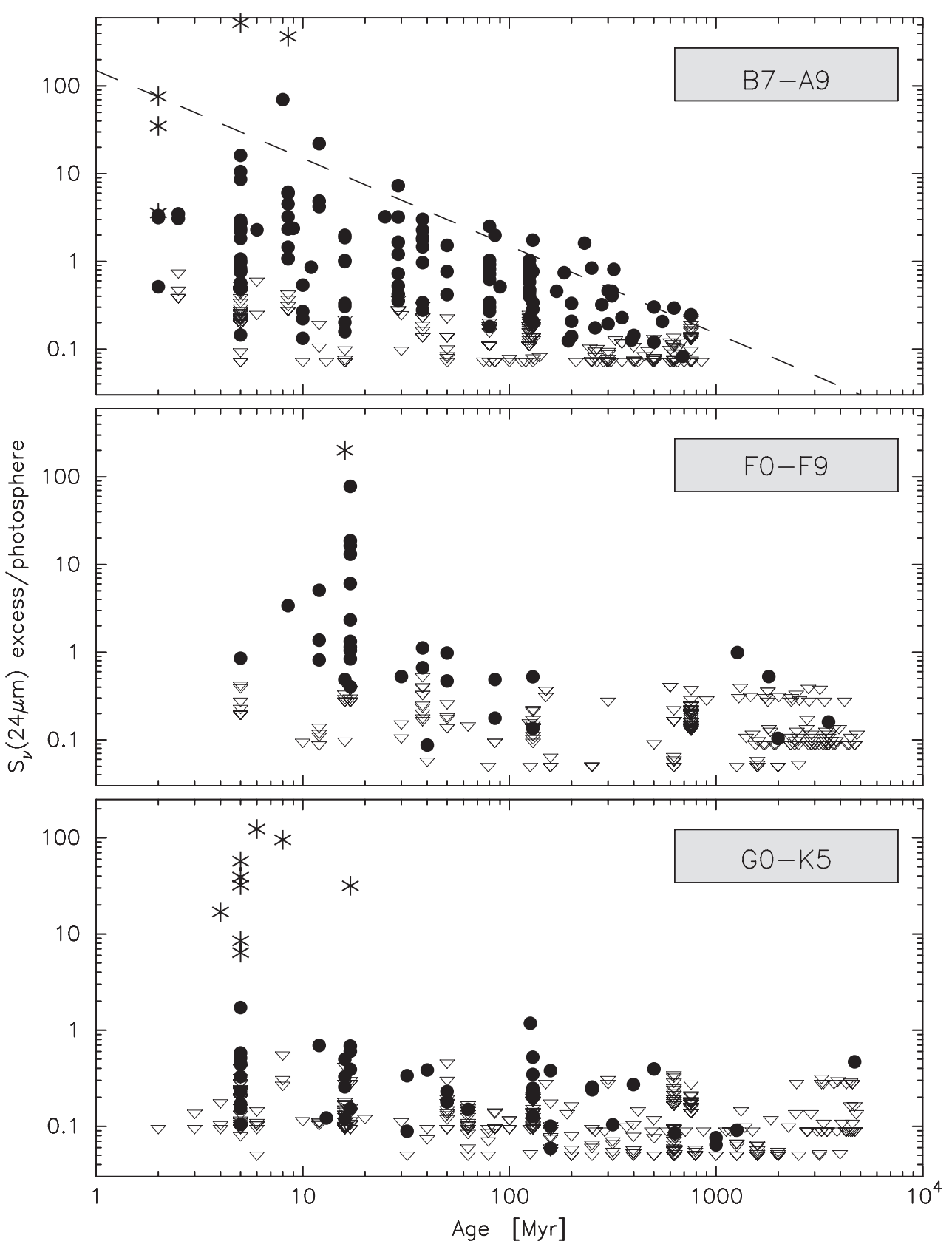

Figure 7. Ratio of the $24 \mu \mathrm{m}$ excess normalized by the photospheric $24 \mu \mathrm{m}$ flux density vs. stellar age compiled from the regions summarized in Table 4 . Filled circles represent stars with a detected $24 \mu \mathrm{m}$ excess that are likely debris disks, and open triangles represent $3 \sigma$ upper limits for stars without excesses. Asterisks indicate optically thick disks (including "anemic" and "evolved" disks). Known Be stars and sources with contaminated $24 \mu$ m photometry have been omitted. The dashed line shows the upper envelope to the $24 \mu \mathrm{m}$ excess from Rieke et al. (2005) for ages older than $5 \mathrm{Myr}$, and extrapolated here to younger ages.

ages of the stellar samples are as young as $2 \mathrm{Myr}$, the younger stars are in the pre-main-sequence phase of evolution and the stellar mass and luminosity will vary with age for each of the spectral type bins. This is particularly true for young $(<10 \mathrm{Myr})$ stars more massive than $\sim 1 M_{\odot}$, which are evolving toward the main-sequence along the Henyey tracks. For example, the spectral type for a $2 M_{\odot}$ star will be K2, G8, and A2 at an age of 2,5 , and $10 \mathrm{Myr}$, respectively according to the Siess et al. (2000) pre-main-sequence evolutionary models and Kenyon \& Hartmann (1995) temperature scale.

Since the blowout size is proportional to $L_{*} / M_{*}$ (see Equation (2)), the minimum grain size in the debris disk will also vary with age. For a fixed spectral type the $L_{*} / M_{*}$ ratio, and hence the blowout particle size, decreases by a factor of $\sim 5$ between $1 \mathrm{Myr}$ and $10 \mathrm{Myr}$ for A0-M0 stars. The total grain surface area is proportional to $a_{\mathrm{min}}^{-0.5}$ for a $a^{-3.5}$ particle size distribution, and the debris luminosity will thus increase in time if the planetesimal belt is in otherwise steady state.

The expected trends were evaluated more quantitatively using the debris disk model described in Section 5. For a given age and spectral type (i.e., stellar temperature), the stellar mass and luminosity were determined from the Siess et al. (2000) evolutionary models for solar metallicity and no convective overshoot. The $24 \mu \mathrm{m}$ debris emission was then computed for a planetesimal belt at an orbital radius of $15 \mathrm{AU}$, with a minimum grain size corresponding to the blowout size. The results are insensitive to the assumed orbital radius since the variations in the debris luminosity with time are caused primarily by changes 


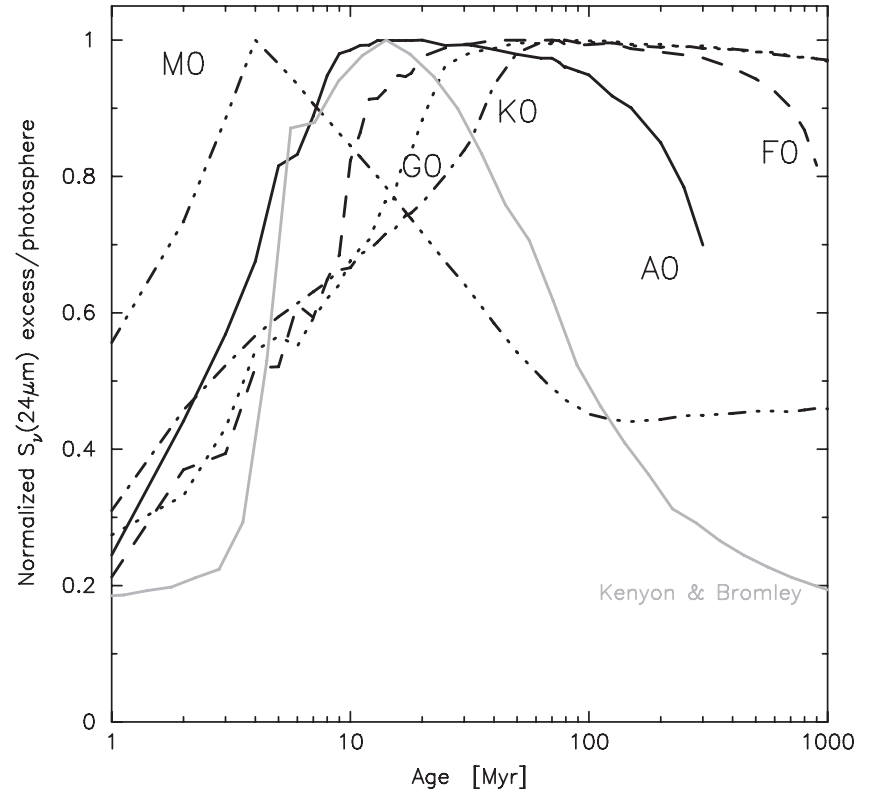

Figure 8. Expected temporal variation in the $24 \mu \mathrm{m}$ excess relative to the stellar photosphere for A0 (solid black curve), F0 (dashed), G0 (dotted), K0 (dash-dot), and M0 stars (dash-dot-dot-dot) stars. The $24 \mu \mathrm{m}$ excess for a given curve has been normalized by the peak excess. The stellar mass and luminosity will vary with age for a fixed spectral type as the star evolves toward the main sequence. The stellar parameters for a given age and spectral type were estimated from the Siess et al. (2000) pre-main-sequence models. The model planetesimal belt is at an orbital radius of $15 \mathrm{AU}$. The debris emission varies in time as stars evolve, which changes the stellar heating and the radiation blowout size. These results show that if the mass of the planetesimal belt is fixed, A-K stars younger than $10 \mathrm{Myr}$ will have a lower $24 \mu \mathrm{m}$ excess since the smaller grains have been blown out of the system by the greater stellar luminosity. For comparison, the solid gray curve shows the variation in the normalized $24 \mu \mathrm{m}$ excess from a planetesimal belt with a mass of the minimum mass solar nebula that extends between 30 and $150 \mathrm{AU}$ around a $2 M_{\odot}$ star with constant stellar luminosity (Kenyon \& Bromley 2008).

in the grain surface area. We assume that the minimum grain size in the debris disk varies instantaneously as stars evolve since the collisional lifetime of dust within an orbital radius of $30 \mathrm{AU}$ is less than $1 \mathrm{Myr}$ for observed debris disks (Backman \& Paresce 1993). In this manner, the variation in the $24 \mu \mathrm{m}$ excess relative to the photosphere was computed considering only stellar evolution.

Figure 8 shows the results of the model calculations. For A0 through $\mathrm{K} 0$ stars, the relative $24 \mu \mathrm{m}$ excess increases by a factor of $\sim 5$ from an age of 1 Myr to a peak at 10-50 Myr. The peak relative excess is reached sooner around earlier spectral types since the stars reach the main sequence on shorter timescales. M-type stars obtain a peak relative excess in only a few million years when the grain blowout size is less than $0.05 \mu \mathrm{m}$. At this point, any grains removed by radiation pressure are inefficient emitters at $24 \mu \mathrm{m}$, and the debris luminosity declines with the stellar luminosity. ${ }^{7}$ If the model assumptions are correct, the sensitivity functions presented in Figure 8 will be superimposed on the evolution of the debris luminosity and must be considered when attributing any increase in the debris luminosity with age to planet formation.

For comparison, the gray solid line in Figure 8 shows the variation in the $24 \mu \mathrm{m}$ excess relative to the photosphere for a

\footnotetext{
7 MIPS observations have yielded a small number of debris disks around young, low-mass T Tauri stars (Cieza et al. 2007). The low excess needs to be interpreted in context of these model calculations, which predict the $24 \mu \mathrm{m}$ excess will be 5 times weaker than a $10 \mathrm{Myr}$ star if the planetesimal belt is otherwise the same.
}

$2 M_{\odot}$ star $(\sim \mathrm{A} 3$ spectral type) with a planetesimal belt between 30 and 150 AU from the Kenyon \& Bromley (2008) models. Since the stellar luminosity is held constant in these models, the temporal variation in the infrared luminosity results from changes in the debris production. The debris evolution in these models depends on the stellar mass and the planetesimal belt properties (mass, orbital radius), but the general prediction is that the fractional debris luminosity rises at early ages, and declines at later times. The results show that for a 30$150 \mathrm{AU}$ planetesimal belt, the rise in the debris luminosity and the age where debris production is maximized resembles the evolutionary curve expected based on stellar evolution alone. Clear differences are observed at older ages where the debris luminosity in the Kenyon \& Bromley (2008) models declines more rapidly.

We now evaluate if the magnitude of the relative $24 \mu \mathrm{m}$ excess varies with stellar age for ages $<20 \mathrm{Myr}$, and if any increase can be attributed to stellar evolution. For both the B7-A9 and G0-K5 spectral-type ranges, any variations in the $24 \mu \mathrm{m}$ excess with age between ages of 5 and 17 Myr have a significance of less than $2 \sigma$ (see Section 6.2 and Figure 7). Thus independent of the selection function presented in Figure 8, no compelling evidence exists for a variation in the mean relative $24 \mu \mathrm{m}$ excess for B7-A9 and G0-K5 stars in that age range.

The F-type stars show the strongest evidence $(2.6 \sigma)$ for a rise in the relative $24 \mu \mathrm{m}$ excess (see Section 6.2), but these results need to be interpreted in view of the expected increase in the debris emission shown in Figure 8. For the F-type stars between ages of 5 and $17 \mathrm{Myr}$, we scaled the magnitude of the $24 \mu \mathrm{m}$ excess by the inverse of the model calculations computed for a F5 star, which corrects for the reduced excess emission expected to occur with stellar evolution. The probability of a trend in the observed excess emission with age (see Figure 7) was then recomputed using the Kendall Tau test. We find the probability that a correlation is not present increases from $p=0.01(2.6 \sigma)$ to $p=0.02(2.3 \sigma)$.

In summary, we conclude that any evidence for an increase in the $24 \mu \mathrm{m}$ debris emission with age between 5 and 17 Myr rests primarily with F-type stars, and the significance is $\lesssim 2.6 \sigma$. Any variations in the relative $24 \mu \mathrm{m}$ excess over this age range for $\mathrm{B} / \mathrm{A}$ and $\mathrm{G} / \mathrm{K}$ are significant at less than $2 \sigma$ confidence. Given the low statistical significance and that the increasing relative excess is observed in a narrow spectral-type range, we hesitate to ascribe the apparent rise in the mean relative $24 \mu \mathrm{m}$ excess among F-stars to the onset of planetesimal stirring.

\subsection{Debris Disks Around M-Stars}

Evolution of debris emission around M-stars is more difficult to quantify since most MIPS surveys are insensitive to the stellar photosphere for these spectral types. Nonetheless, for completeness, we present in Figure 9 the $24 \mu \mathrm{m}$ excess relative to photosphere as a function of age for M0-M5 stars in IC 348 (Lada et al. 2006), $\sigma$ Ori (Hernández et al. 2007), Upper Sco, $\eta$ Cha (Gautier et al. 2008), TW Hydra moving group (Low et al. 2005), NGC 2547 (Forbrich et al. 2008), and IC 2391 (Siegler et al. 2007). M-stars with upper limits to the $24 \mu \mathrm{m}$ excess are not shown for clarity, and because most studies have not reported upper limits if a star is not detected.

Figure 9 again illustrates the dichotomy at young ages between classified primordial disks (asterisks) and debris disks (filled circles). The $24 \mu \mathrm{m}$ excess around Upper Sco M-stars is comparable to many of the stars in NGC 2547 at an age of 38 Myr (Naylor \& Jeffries 2006). However, five M-stars in 


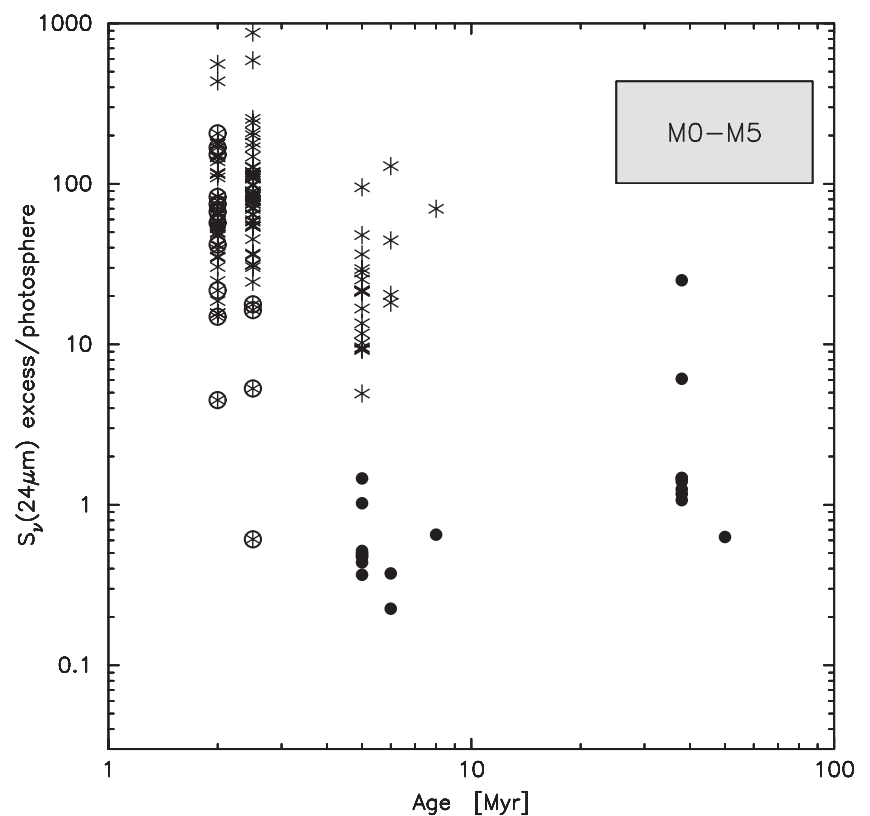

Figure 9. $24 \mu \mathrm{m}$ excess for M0-M5 stars as a function of stellar age. Excess sources are shown for IC 348 (age 2 Myr; Lada et al. 2006), $\sigma$ Ori (2.5 Myr; Hernández et al. 2007), Upper Sco (5 Myr), $\eta$ Cha (6 Myr; Gautier et al. 2008), TW Hydra moving group (8 Myr; Low et al. 2005), NGC 2547 (38 Myr; Forbrich et al. 2008), and IC 2391 (50 Myr; Siegler et al. 2007). Asterisks indicate stars where the $24 \mu \mathrm{m}$ excess is thought to originate from an optically thick disk; circled asterisks are optically thick disks further subdivided as "anemic" or "evolved" disks. Filled circles indicate stars classified as debris systems. M-stars with upper limits at $24 \mu \mathrm{m}$ have been omitted for clarity. Not all surveys shown here are sensitive to the stellar photosphere, and this diagram should not be used to infer the evolution of debris emission around M-type stars.

NGC 2547 have a $24 \mu \mathrm{m}$ excess of $\sim 10$ times the photosphere; three of these five stars have been observed with IRAC (Young et al. 2004), and one has an $8 \mu \mathrm{m}$ excess. By contrast, all of the Upper Sco sources classified with a primordial disk have an $8 \mu \mathrm{m}$ excess. It would be unusual for an optically thick disk to persist for $\sim 38 \mathrm{Myr}$, and the debris disk is a reasonable interpretation for the NGC 2547 sources. These results suggest that distinguishing primordial disks from debris disks for M-type stars merits a more detailed examination (cf. Ercolano et al. 2009).

\section{SUMMARY}

We have presented the results of a Spitzer $24 \mu \mathrm{m}$ and $70 \mu \mathrm{m}$ photometric survey of 205 members of the Upper Scorpius OB association. These data were combined with MIPS photometry for 15 Upper Sco sources observed by the FEPS Legacy program to provide a census of circumstellar disks around stars with spectral ranges ranking from $\mathrm{B} 0$ to $\mathrm{M} 5$ at an association age of 5 Myr (Preibisch et al. 2002). By analyzing the $24 \mu \mathrm{m}$ photometry with $4.5 \mu \mathrm{m}$ and $8 \mu \mathrm{m}$ measurements presented in Paper I, we identify 54 stars that have observed $24 \mu \mathrm{m}$ emission that exceeds the expected stellar photospheric emission by $32 \%$ or more. Similarly, 12 stars were identified with a $\geqslant 3 \sigma$ photometric excess at $70 \mu \mathrm{m}$; all 12 of these stars have a detectable $24 \mu \mathrm{m}$ excess.

The nature of the excess sources was established based on the color and magnitude of the excess emission. We find a dichotomy of excess characteristics. One population, found only around the $\mathrm{K}$ - and M-type stars, has strong excess emission at both $8 \mu \mathrm{m}$ and $24 \mu \mathrm{m}$ that is comparable to known optically thick circumstellar disks. A second population, found around all spectral types, has weak $8 \mu \mathrm{m}$ and $24 \mu \mathrm{m}$ excesses compared to optically thick and "anemic" disks in the IC 348 cluster (Lada et al. 2006). We suggest that these weak excesses originate from debris disks formed from the collisional shattering of planetesimals, although we cannot exclude the possibility that these systems are the remnants of optically thick disks. Of the 54 excess stars, we attribute the $24 \mu \mathrm{m}$ excess emission to two Be stars, 24 primordial disks, and 28 debris disks. The debris disks include $11 \mathrm{~K} / \mathrm{M}$ stars.

The debris disks were analyzed to investigate whether the orbital radius of the presumed planetesimal belts vary systematically with spectral type. We modeled the emission with a power-law distribution of grain sizes following the Dohnanyi (1969) equilibrium distribution $\left(n(a) \propto a^{-3.5}\right)$. The orbital radius $(R)$ and grain size distribution $\left(N_{\mathrm{o}}\right.$; see Equation (1)) were set to reproduce the observed excess emission around nine $\mathrm{B} / \mathrm{A}$ stars with infrared excesses at both $16 \mu \mathrm{m}$ and $24 \mu \mathrm{m}$. The expected emission around stars of other spectral types were then estimated after allowing for variations in the stellar heating and radiation blowout of small grains. We find that the magnitude of the $24 \mu \mathrm{m}$ emission observed around later stars can be produced by this model, indicating it is not necessary to invoke a different radial distribution. This model predicts a steep fall off in the relative $24 \mu \mathrm{m}$ excess for stars earlier than $\sim \mathrm{B} 7$ due to blowout of the small grains, and later than $\sim \mathrm{M} 0$ due to reduced stellar heating.

The Upper Sco results are combined with other Spitzer $24 \mu \mathrm{m}$ surveys in the literature to reassess the evolution of $24 \mu \mathrm{m}$ debris emission. After consideration of both sample sizes and detection limits, we find a decline in the magnitude of the $24 \mu \mathrm{m}$ excess relative to the photosphere for spectral types between $\mathrm{B} 7$ and $\mathrm{K} 5$ and ages between $10 \mathrm{Myr}$ and $5 \mathrm{Gyr}$, as has been noted in previous studies (Habing et al. 2001; Decin et al. 2003; Rieke et al. 2005; Carpenter et al. 2009). We also investigated if the $24 \mu \mathrm{m}$ excess increases with stellar age for ages $\lesssim 20 \mathrm{Myr}$. Such an increase may be indicative of the onset of planetesimal stirring (Kenyon \& Bromley 2008). We caution, however, that a similar increase in the excess emission may also result from premain-sequence evolution; for a fixed spectral type, the $L_{*} / M_{*}$ ratio will decrease with age, which leads to a higher debris luminosity as fewer grains are ejected by radiative forces. The observed mean $24 \mu \mathrm{m}$ excess around F-type stars increases between ages of 5 and $17 \mathrm{Myr}$ as previously found by Currie et al. (2008a, see also Hernández et al. 2006), but with a significance of $\sim 2.3 \sigma-2.6 \sigma$ confidence. The variations in the mean $24 \mu \mathrm{m}$ excess around $\mathrm{B} / \mathrm{A}$ and $\mathrm{G} / \mathrm{K}$ stars over the same age range are significant at less than $2 \sigma$ confidence. We conclude that the observational data do not yet require a physical mechanism to produce a peak in the observed debris emission.

We are grateful to Scott Kenyon for his comments on the manuscript. This work is based on observations made with the Spitzer Space Telescope, which is operated by JPL/Caltech under a contract with NASA. Support for this work was provided by NASA through an award issued by JPL/Caltech. This research made use of the SIMBAD database, the IRSA archive, and the Two Micron All Sky Survey.

\section{APPENDIX}

\section{COMPILATION OF SPITZER DEBRIS DISK SURVEYS}

To compare the Upper Sco results with previous observations, we compiled data from published Spitzer $24 \mu \mathrm{m}$ surveys 
Table 4

Compilation of Debris Disk Surveys

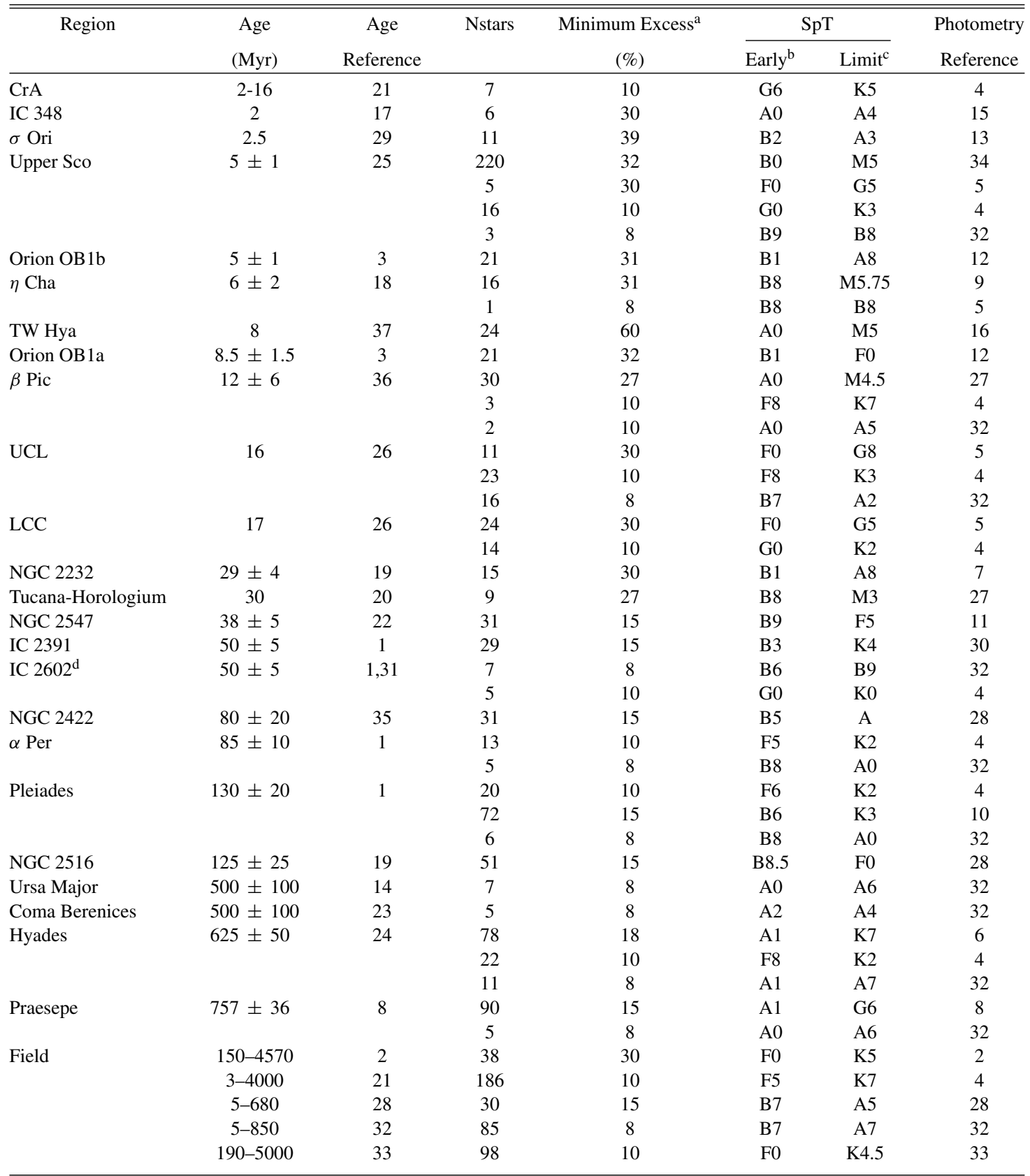

Notes.

a The minimum percent $24 \mu \mathrm{m}$ flux density above the photosphere used to identify an excess.

b Earliest spectral type in the survey.

${ }^{c}$ Latest spectral type where the minimum excess could be detected.

${ }^{\mathrm{d}}$ We adopt an age of $50 \mathrm{Myr}$ for IC 2391, and assume IC 2391 and IC 2602 have the same age following Stauffer et al. (1997).

References. (1) Barrado y Navascués et al. 2004; (2) Beichman et al. 2006; (3) Briceño et al. 2005; (4) Carpenter et al. 2008; (5) Chen et al. 2005; (6) Cieza et al. 2008; (7) Currie et al. 2008b; (8) Gaspar et al. 2009; (9) Gautier et al. 2008; (10) Gorlova et al. 2006; (11) Gorlova et al. 2007; (12) Hernández et al. 2006; (13) Hernández et al. 2007; (14) King et al. 2003; (15) Lada et al. 2006; (16) Low et al. 2005; (17) Luhman et al. 2003; (18) Luhman \& Steeghs 2004; (19) Lyra et al. 2006; (20) Mamajek et al. 2004; (21) Meyer et al. 2006; (22) Naylor \& Jeffries 2006; (23) Odenkirchen et al. 1998; (24) Perryman et al. 1998; (25) Preibisch et al. 2002; (26) Preibisch \& Mamajek 2008; (27) Rebull et al. 2008; (28) Rieke et al. 2005; (29) Sherry et al. 2008; (30) Siegler et al. 2007; (31) Stauffer et al. 1997; (32) Su et al. 2006; (33) Trilling et al. 2008; (34) This study; (35) van Rensbergen et al. 1978; (36) Zuckerman et al. 2001; (37) Zuckerman \& Song 2004.

for debris disks. We considered studies in which the parent sample is not biased for or against the presence of a debris disk. This requirement implies that the parent sample must have been selected by photospheric indicators, and that the
Spitzer observations were sensitive to the stellar photosphere. In practice, this is not strictly true for nearby moving group such as $\beta$ Pic, $\eta$ Cha, and TW Hydra, where the moving group was initially identified based on the presence of an 
apparently isolated sources with an prominent infrared excess. We nonetheless include these groups since the subsequent surveys for moving group members were not biased toward circumstellar disks.

Table 4 summarizes the surveys that were compiled and contain five stars or more. We include in this table the name of the region, the adopted age, the earliest spectral type in the survey, the latest spectral type at which the photosphere can be reliable detected as discussed above, the number of stars in this spectral-type range, and the minimum $24 \mu \mathrm{m}$ excess above the stellar photosphere that could be detected. In some regions (e.g., NGC 2232, Pleiades, Praesepe), not all stars have spectral types available from spectroscopic observations, and the spectral type was estimated from optical or near-infrared colors. Field stars with ages between $3 \mathrm{Myr}$ and $5 \mathrm{Gyr}$ were compiled from the FEPS survey of solar-type stars (Meyer et al. 2006), the survey of solar-type field stars by Beichman et al. (2006) and Trilling et al. (2008), and the survey of B/A stars by Rieke et al. (2005) and Su et al. (2006).

The photometry was extracted from the publications listed in Table 4. No attempt was made to re-reduce the data under a common data reduction method or to re-assess cluster or association membership. Several studies noted sources where the photometry was contaminated by nebulosity or a companion sources. If these stars were excluded from the analysis in the original study, they were also excluded in our analysis. After compiling the source lists, we ran all source names through SIMBAD to identify duplicate sources appearing under different names. If photometry appear for a star in more than one survey, we adopted the photometry from the survey with the higher signal to noise.

Most Spitzer studies have identified sources with excesses based on the presence of unusually red infrared colors (e.g., [K]-[24], [8]-[24]). Each study has a minimum detectable excess that depends on the depth of the survey, the presence of nebulosity in sky background, the distance to the stellar sample, and the intrinsic brightness of the star. In nearly all cases, we adopted the limiting thresholds adopted in the original study to identify $24 \mu \mathrm{m}$ excesses, which is normally taken to be three or four times the rms scatter around the main locus of data points in a color-color diagram or a stellar color that is thought to represent photospheric colors (see, e.g., Figure 1). We then identified the latest spectral type in which the internal uncertainty in the measured photospheric flux was less than this rms scatter. This criteria removed sources where the photometric uncertainty was comparable to the excess being measured. If the threshold to identify an excess $(\equiv \Delta)$ was expressed in magnitudes, the adopted threshold was estimated as $10^{\Delta / 2.5}$.

The ages for clusters and associations were adopted from the literature based on a number of techniques, including pre-mainsequence evolutionary tracks, lithium depletion, and the main sequence turnoff point. The age uncertainties were adopted from the literature, but if no uncertainty was quoted, we assumed an age uncertainty of 0.3 dex in the logarithm of the age. The age uncertainties for the clusters and associations do not reflect any systematic uncertainty associated with the particular method. The ages for field stars were adopted from the studies presenting the Spitzer data. The references for the ages are provided in Table 4.

A few studies are not included in this comparison since the observations could not be analyzed in a consistent manner. First, we did not include surveys where the observations were not sensitive to the photosphere for spectral types A or later, either because the regions are more distant than $\sim 900$ pc (Trumpler 37 and NGC 7160, Sicilia-Aguilar et al. 2006; h \& $\chi$ Persei, Currie et al. 2008a; NGC 2262, Currie et al. 2008b), or the observations were exclusively of late spectral types ( $\lambda$ Orionis; Barrado y Navascués et al. 2007). MIPS $24 \mu \mathrm{m}$ observations of the $\gamma$ Velorum cluster were not incorporated; while the observations were sensitive to the photosphere for stars early than $\sim F 8$, the contamination from field stars is non-negligible (Hernández et al. 2008). We also did not analyze MIPS observations of weak-lined T Tauri stars since the samples excluded optically thick disks (Padgett et al. 2006; Cieza et al. 2007).

Given the heterogeneous nature of the analyzes to identify Spitzer excesses, we occasionally had to re-analyze data from the original study to provide a uniform analysis. The following discussion summarizes the regions where we re-interpreted the observations.

$\sigma$ Orionis. From the photometry presented by Hernández et al. (2007), 97\% (33 out of 34) stars brighter than $J=10.2$ mag were detected at $24 \mu \mathrm{m}$, while only four of seven stars between $J=$ 10.2 and $10.5 \mathrm{mag}$ were detected. The completeness limit was then defined as $J=10.2$. For stars fainter than $J=10.2$, the bluest star has a dereddened color of $J-H=0.15$. Therefore, we assumed the survey is complete for $J-H<=0.15$, corresponding to spectral type earlier than F0.

IC 348. Of the eight A-type stars in IC 348 studied by Lada et al. (2006), six were detected by at $24 \mu \mathrm{m}$, one was not observed at $24 \mu \mathrm{m}$, and one has an upper limit from locally bright background. Only $57 \%$ of the FG stars and less than $33 \%$ of the KM stars in IC 348 have $24 \mu \mathrm{m}$ measurements, and therefore only the A-stars in IC 348 were included. We adopted a detection threshold of $30 \%$ for a $24 \mu \mathrm{m}$ excess based on Figure 7 in Lada et al. (2006).

$N G C$ 2232. Currie et al. (2008b) estimated a $5 \sigma$ and $10 \sigma$ detection limit of $10.5 \mathrm{mag}$ and $9.75 \mathrm{mag}$ for their $24 \mu \mathrm{m}$ survey. They also find that the number of sources detected at $24 \mu \mathrm{m}$ falls rapidly for sources fainter than [24] $=10.5$. We therefore adopted [24] $=10 \mathrm{mag}$ as a limiting magnitude, and find that $99 \%$ of stars brighter than $J=10$ mag have a MIPS $24 \mu \mathrm{m}$ detection brighter than this limit. For a distance of 340 pc, an age of $25 \mathrm{Myr}$, and a color excess of $E(B-V)=0.07$, $J=10$ corresponds roughly to a A8 star, which we adopt as the completeness limit of the survey. The rms scatter in the $K-$ [24] color for stars brighter than the completeness limit and not containing an infrared excess (as defined in Currie et al. 2008b) is 0.09 mag, which was adopted as the photometric uncertainty.

Praesepe. From Figure 6 in Gaspar et al. (2009), we find that the number of stars with unphysical blue stellar colors increases for stars redder than $r-K_{\mathrm{s}}=1.5$. We consider only stars bluer than this limit, which corresponds to stars earlier than G5.

\section{REFERENCES}

Backman, D. E., \& Paresce, F. 1993, in Protostars and Planets III, ed. E. H. Levy \& K. L. Lunine (Tucson, AZ: Univ. Arizona Press), 1253

Barrado y Navascués, D., Stauffer, J. R., \& Jayawardhana, R. 2004, ApJ, 614, 368

Barrado y Navascués, D., et al. 2007, ApJ, 664, 481

Beichman, C. A., et al. 2006, ApJ, 652, 1674

Briceño, C., Calvet, N., Hernández, J., Vivas, A. K., Hartmann, L., Downes, J. J., \& Berlind, P. 2005, AJ, 129, 907

Bryden, G., et al. 2006, ApJ, 636, 1098

Bouy, H., \& Martín, E. L. 2009, A\&A, 504, 981

Burns, J. A., Lamy, P. L., \& Soter, S. 1979, Icarus, 40, 1

Cardelli, J. A., Clayton, G. C., \& Mathis, J. S. 1989, ApJ, 345, 245 
Carpenter, J. M., Mamajek, E. E., Hillenbrand, L. A., \& Meyer, M. R. 2006, ApJ, 651, L49 (Paper I)

Carpenter, J. M., et al. 2008, ApJS, 179, 423

Carpenter, J. M., et al. 2009, ApJS, 181, 197

Chapman, N. L., Mundy, L. G., Lai, S., \& Evans, N. J. 2009, ApJ, 690, 496

Chen, C. H., Jura, M., Gordon, K. D., \& Blaylock, M. 2005, ApJ, 623, 493

Cieza, L. A., Cochran, W. D., \& Augereau, J.-C. 2008, ApJ, 679, 720

Cieza, L. A., et al. 2007, ApJ, 667, 308

Crampton, D. 1968, AJ, 73, 337

Currie, T., \& Kenyon, S. J. 2009, AJ, 138, 703

Currie, T., Kenyon, S. J., Balog, Z., Rieke, G., Bragg, A., \& Bromley, B. 2008a, ApJ, 672, 558

Currie, T., Lada, C. J., Plavchan, P., Robitaille, T. P., Irwin, J., \& Kenyon, S. J. 2009, ApJ, 698, 1

Currie, T., Plavchan, P., \& Kenyon, S. J. 2008b, ApJ, 688, 597

Dahm, S., \& Carpenter, J. M. 2009, AJ, 137, 4024

Decin, G., Dominik, C., Waters, L. B. F. M., \& Waelkens, C. 2003, ApJ, 598, 636

de Zeeuw, P. T., Hoogerwerf, R., de Bruijne, J. H. L., Brown, A. G. A., \& Blaauw, A. 1999, AJ, 117, 354

Dohnanyi, J. W. 1969, J. Geophys. Res., 74, 2531

Dominik, C., \& Decin, G. 2003, ApJ, 598, 626

Engelbracht, et al. 2007, PASP, 119, 994

Ercolano, B., Clarke, C. J., \& Robitaille, T. P. 2009, MNRAS, 394, L141

Espaillat, C., Calvet, N., D’Alessio, P., Hernández, J., Qi, C., Hartmann, L., Furlan, E., \& Watson, D. M. 2007, ApJ, 670, L135

Forbrich, J., Lada, C. J., Muench, A. A., \& Teixera, P. S. 2008, ApJ, 687, 1107

Gaspar, A., et al. 2009, ApJ, 697, 1578

Gautier, T. N., III, Rebull, L. M., Stapelfeldt, K. R., \& Mainzer, A. 2008, ApJ, 683,813

Gautier, T. N., III, et al. 2007, ApJ, 667, 527

Gorlova, N., Balog, Z., Rieke, G. H., Muzerolle, J., Su, K. Y. L., Ivanov, V. D., \& Young, E. T. 2007, ApJ, 670, 516

Gorlova, N., et al. 2006, ApJ, 649, 1028

Gustafson, B. A. S. 1994, Ann. Rev. Earth Planetary Sci., 22, 553

Habing, H. J., et al. 2001, A\&A, 365, 545

Hernández, J., Calvet, N., Hartmann, L., Briceño, C., Sicilio-Aguilar, A., \& Berlind, P. 2005, AJ, 129, 856

Hernández, J., Hartmann, L., Calvet, N., Jeffries, R. D., Gutermuth, R., Muzerolle, J., \& Stauffer, J. 2008, ApJ, 686, 1195

Hernández, J., et al. 2006, ApJ, 652, 472

Hernández, J., et al. 2007, ApJ, 662, 1067

Holland, W. S., et al. 1998, Nature, 392, 788

Houk, N. 1982, Michigan Catalogue of Two-Dimensional Spectral Types for the HD Stars, Vol. 3 (Ann Arbor, MI: Univ. Michigan)

Houk, N., \& Smith-Moore, M. 1988, Michigan Catalogue of Two-Dimensional Spectral Types for the HD stars, Vol. 4 (Ann Arbor, MI: Univ. Michigan)

Jaschek, C., Jaschek, M., \& Kucewicz, B. 1964, Z. Astrophys., 59, 108

Kenyon, S. J., \& Bromley, B. C. 2004, AJ, 127, 513

Kenyon, S. J., \& Bromley, B. C. 2008, ApJS, 179, 451

Kenyon, S. J., \& Hartmann, L. 1995, ApJ, 101, 117

King, J., Villarreal, A. R., Soderblom, D. R., Gulliver, A. F., \& Adelman, S. J. 2003, AJ, 125, 1980

Köhler, Kunkel M., Leinert, C., \& Zinnecker, H. 2000, A\&A, 356, 541

Kunkel, M. 1999, PhD thesis, Julius-Maximilians-Universität Würzburg

Lada, C. J., et al. 2006, AJ, 131, 1574

Lagrange, A., Backman, D. E., \& Artymowicz, P. 2000, in Protostars and Planets IV, ed. V. Mannings, A. Boss, \& S. S. Russell (Tucson, AZ: Univ. Arizona Press), 639

Lavalley, M., Isobe, T., \& Feigelson, E. 1992, in ASP Conf. Ser. 25, Astronomical Data Analysis Software and Systems I, ed. D. M. Worrall, C. Biemesderfer, \& J. Barnes (San Francisco, CA: ASP), 245

Liou, J.-C., \& Zook, H. A. 1999, AJ, 118, 580

Löhne, T., Krivov, A. V., \& Rodmann, J. 2008, ApJ, 673, 1123

Low, F. J., Smith, S., Werner, M., Chen, C., Krause, V., Jura, M., \& Hines, D. C. $2005, \mathrm{ApJ}, 631,1170$

Luhman, K. L., Stauffer, J. R., Muench, A. A., Rieke, G. H., Lada, E. A., Bouvier, J., \& Lada, C. J. 2003, ApJ, 493, 1093

Luhman, K. L., \& Steeghs, D. 2004, ApJ, 609, 917

Lyra, W., Moitinho, A., van der Bliek, N. S., \& Alves, J. 2006, A\&A, 453, 101

Makovoz, D., \& Marleau, F. R. 2005, PASP, 117, 1113
Mamajek, E. E., Meyer, M. R., Hinz, P. M., Hoffmann, W. F., Cohen, M., \& Hora, J. L. 2004, ApJ, 612, 496

Martín, E. L. 1998, AJ, 115, 351

Mathis, J. S. 1990, ARA\&A, 28, 37

Meyer, M. R., Backman, D. E., Weinberger, A. J., \& Wyatt, M. C. 2007, in Protostars and Planets V, ed. B. Reipurth, D. Jewitt, \& K. Keil (Tucson, AZ: Univ. Arizona Press), 573

Meyer, M. R., et al. 2006, PASP, 118, 1690

Meyer, M. R., et al. 2008, ApJ, 673, 181

Moór, A., Ábrahám, P., Derekas, A., Kiss, Cs., Kiss, L. L., Apai, D., Grady, C., \& Henning, Th. 2006, ApJ, 644, 525

Najita, J., \& Williams, J. P. 2005, ApJ, 635, 625

Naylor, T. 1998, MNRAS, 296, 339

Naylor, T., \& Jeffries, R. D. 2006, MNRAS, 373, 1251

Odenkirchen, M., Soubiran, C., \& Colin, J. 1998, New Astron., 3, 583

Odenwald, S. F. 1986, ApJ, 307, 711

Padgett, D. L., et al. 2006, ApJ, 645, 1283

Papovich, C., et al. 2004, ApJS, 154, 70

Pascucci, I., et al. 2006, ApJ, 651, 1177

Perryman, M. A. C., et al. 1998, A\&A, 331, 81

Plavchan, P., Jura, M., \& Lipscy, S. J. 2005, ApJ, 631, 1161

Plavchan, P., et al. 2009, ApJ, 698, 1068

Porter, J. M., \& Rivinius, T. 2003, PASP, 115, 1153

Preibisch, T., Brown, A. G. A., Bridges, T., Guenther, E., \& Zinnecker, H. 2002, AJ, 124, 404

Preibisch, T., Guenther, E., \& Zinnecker, H. 2001, AJ, 121, 1040

Preibisch, T., Guenther, E., Zinnecker, H., Sterzik, M., Frink, S., \& Roeser, S. 1998, A\&A, 333, 619

Preibisch, T., \& Mamajek, E. 2008, in Handbook of Star Forming Regions, Vol. II, ed. Bo Reipurth (San Francisco, CA: ASP), 235

Preibisch, T., \& Zinnecker, H. 1999, AJ, 117, 2381

Rebull, L. M., et al. 2008, ApJ, 681, 1484

Rhee, J., Song, I., Zuckerman, B., \& McElwain, M. 2007, ApJ, 660, 1556

Rieke, G. H., et al. 2005, ApJ, 620, 1010

Scholz, A., Jayawardhana, R., Wood, K., Meeus, G., Stelzer, B., Walker, C., \& O’Sulliivan, M. 2007, ApJ, 660, 1517

Sherry, W. H., Walter, F. M., Wolk, S. J., \& Adams, N. R. 2008, AJ, 135, 1616 Sicilia-Aguilar, A., et al. 2006, ApJ, 638, 897

Siegler, N., et al. 2007, ApJ, 654, 580

Siess, L., Dufour, E., \& Forestini, M. 2000, A\&A, 358, 593

Skrutskie, M. F., et al. 2006, AJ, 131, 1163

Spangler, C., Sargent, A. I., Silverstone, M. D., Becklin, E. E., \& Zuckerman, B. 2001, ApJ, 555, 932

Stauffer, J. R., et al. 1997, ApJ, 479, 776

Strom, K. M., Strom, S. E., Edwards, S., Cabrit, S., \& Skrutskie, M. F. 1989, AJ, 97,1451

Strubbe, L. E., \& Chiang, E. I. 2006, ApJ, 648, 652

Su, K. Y. L., et al. 2006, ApJ, 653, 675

Takeuchi, T., \& Artymowicz, P. 2001, ApJ, 557, 990

Trilling, D. E., et al. 2008, ApJ, 674, 1086

van der Bliek, N. S., Manfroid, J., \& Bouchet, P. 1996, A\&AS, 119, 547

van Rensbergen, W., Hammerschlag-Hensberge, G., \& van den Heuvel, E. P. J. 1977, A\&A, 64, 131

Walter, F. M., Vrba, F. J., Mathieu, R. D., Brown, A., \& Myers, P. C. 1994, AJ, 107, 692

Weingartner, J. C., \& Draine, B. T. 2001, ApJ, 548, 296

Werner, M., Fazio, G., Rieke, G., Roellig, T. L., \& Watson, D. M. 2006, ARA\&A, 44, 269

Werner, M., et al. 2004, ApJS, 154, 1

Williams, D. R., \& Wetherill, G. W. 1994, Icarus, 107, 117

Wood, B. E. 2004, Living Rev. Solar Phys., 1, 2

Wyatt, M. C. 2003, ApJ, 598, 1321

Wyatt, M. C. 2008, ARA\&A, 46, 339

Wyatt, M. C., Smith, R., Greaves, J. S., Beichman, C. A., Bryden, G., \& Lisse, C. M. 2007a, ApJ, 658, 569

Wyatt, M. C., Smith, R., Su, K. Y. L., Rieke, G. H., Greaves, J. S., Beichman, C. A., \& Bryden, G. 2007b, ApJ, 663, 365

Young, E. T., et al. 2004, ApJS, 154, 428

Zuckerman, B., Forveille, T., \& Kastner, J. H. 1995, Nature, 373, 494

Zuckerman, B., \& Song, I. 2004, ARA\&A, 42, 685

Zuckerman, B., Song, I., Bessell, M. S., \& Webb, R. A. 2001, ApJ, 562, L87 